\title{
ANTICIPATING MOTHERING IN THE \\ MEXICAN ORIGIN WOMAN
}

by

\author{
SUSAN M. BAXLEY \\ Presented to the Faculty of the Graduate School of \\ The University of Texas at Arlington in Partial Fulfillment \\ of the Requirements \\ for the Degree of \\ DOCTOR OF PHILOSOPHY
}

THE UNIVERSITY OF TEXAS AT ARLINGTON

December 2008 
Copyright @ by Susan M. Baxley 2008

All Rights Reserved 


\section{ACKNOWLEDGEMENTS}

To my mother and father, who gave me so much and were always proud of me, no matter what I decided to do next. To my wonderful family for their support and encouragement, even though they often times did not understand why I would want to return to school.

My children (Sean and Angela, Erika and David) and my grandchildren (Evan, Cameron, Tyler, and Aaron), I appreciate how you helped me by giving me space, help with editing and computer programs, but especially for providing your Nana wonderful distractions, love, and laughs.

To my husband, Phil. He has been the one who has always encouraged me, and when I thought I could not do any more, told me to do it. He has always believed I could do more than I thought I could.

To the Mexican origin women who were willing to participate in this study and share their thoughts and feelings. Without you, this study would not have happened. To Silvia Urueta and Juanita Garcia who provided help with interviews and finding the women for the study.

Most of all, I want to thank Dr. Mary Lou Bond for her guidance, encouragement, and patience during the process of writing my dissertation. All the tense changes about drove us both crazy. You were always there for me. I also want to thank her for suggesting that I start the program and mentoring me through to completion. I could not ask for a better mentor.

I also want to thank Dr. Carolyn Cason for giving me a job and allowing me to learn and make mistakes. You have been a wonderful inspiration and have provided great opportunities to help me grow as a scholar. 
A sincere thank you goes to Dr. Diane Snow for working with me and offering suggestions before and during this process. To Dr. Jennifer Gray, thank you for the encouragement along the way especially in the beginning when I did not know what I was doing. To Dr. Donelle Barnes for sharing her knowledge of qualitative research and agreeing to review and code two of the interviews providing credibility to my dissertation. To Dr. Ferne Kyba for honoring me with the Ferne Kyba Fellowship to assist with financial assistance to complete my dissertation.

To all my great friends whom I have made; we could not have done it without each other. We have shared joys, sorrows, frustrations, but most of all some great times together. I will treasure this time we have had together. For those still to come, it will happen!

The faculty and staff at the University of Texas at Arlington have given me a warm place to grow, provided encouragement, and shared in the joys of my success. I could not ask for a better place to grow and become a nurse scientist.

November 13, 2008 


\section{ABSTRACT \\ ANTICIPATING MOTHERING IN THE MEXICAN ORIGIN WOMAN \\ Susan M. Baxley, PhD}

The University of Texas at Arlington, 2008

Supervising Professor: Mary Lou Bond, PhD

Not all women experience pregnancy and motherhood in the same way. There needs to be a greater understanding of how Mexican origin women experience pregnancy and anticipate mothering since Hispanics comprise the largest and fastest growing minority group in the United States. The purpose of this qualitative, descriptive inquiry was to explore the meaning of attachment, commitment, and preparation during pregnancy as an anticipatory phase of mothering for the Mexican origin woman giving birth in the United States. Culture, the partner, family and friends were also explored as to how they influenced the process of anticipating mothering. Semi-structured interviews provided direct, in-depth responses about the woman's experiences, perceptions, feelings, and knowledge of becoming a mother. The richness of the results was highlighted in the statements of the young women reflecting on their pregnancy and anticipation of becoming a mother. The sample consisted of self-identifying Mexican origin primigravidas of $23-30$ weeks gestation at the time of the interview. They were between $18-23$ years of age, monolingual English, or bilingual-English/Spanish. The findings reflect the components of attachment, commitment, and preparation, three concepts of the model, Anticipating Mothering in the Mexican origin Woman. Three-emergent themes were identified from the interviews; determination to succeed, strong woman, and good mother. These themes 
appear to be interrelated and separation may not be possible with this group of women. Nurses and other health care providers need to be aware of the cultural patterns of this group of women during pregnancy. As noted by other researchers, nurses need to capitalize on the strength of these women instead of their deficits when providing care for them. Because of the importance of the other women in the expectant woman's life, suggestions for changes in formal prenatal classes as the primary mode of preparation for birth and parenting need to be considered. Services need to support their existing resiliency (determination and strength). Intergenerational and interventional research is needed to learn how to be more attuned to the beliefs and thoughts of this group of women and design appropriate programs of prenatal care and education for them. 


\section{TABLE OF CONTENTS}

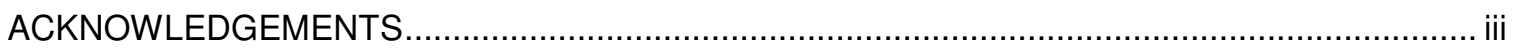

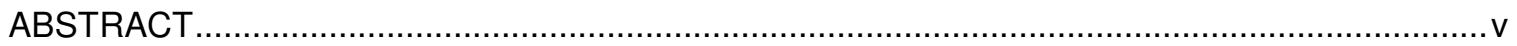

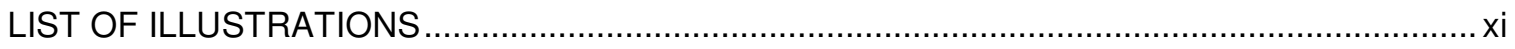

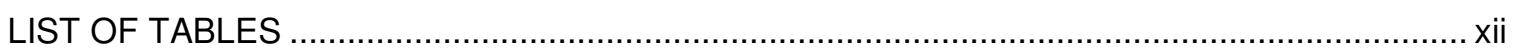

$\begin{array}{ll}\text { Chapter } & \text { Page }\end{array}$

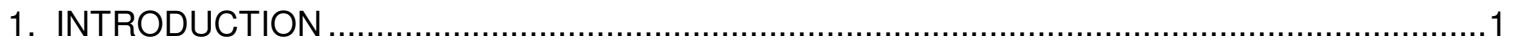

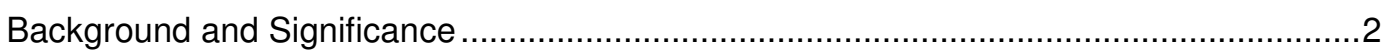

Philosophical/Theoretical Perspective ......................................................... 5

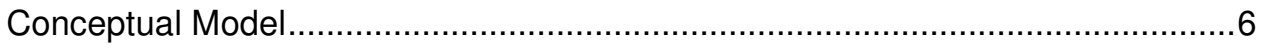

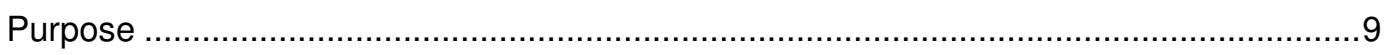

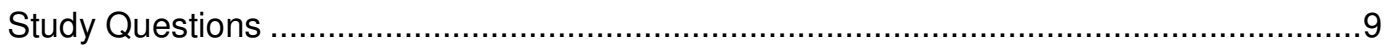

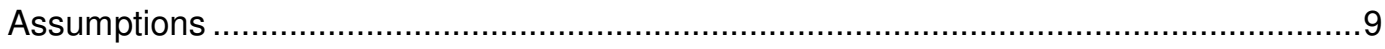

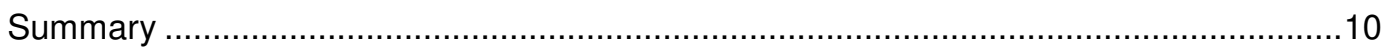

2. CRITICAL REVIEW OF RELEVANT LITERATURE ....................................................11

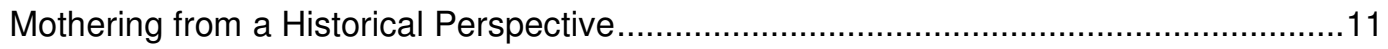

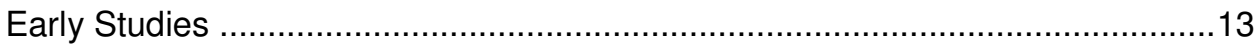

Development of Mercer's Original Theory .................................................17

Mercer's Revised Theory, Becoming a Mother ..............................................19

Psychosocial Pregnancy: Commitment, Attachment, and Preparation ......................20

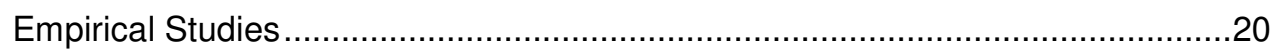

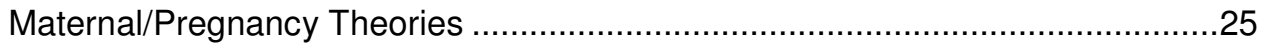

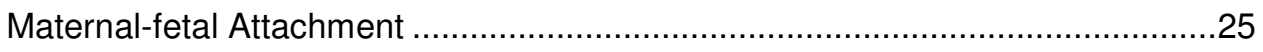


Culture

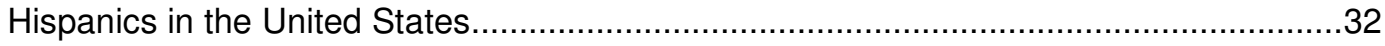

Hispanic Culture Values/Family, Pregnancy, and Mothering ......................................33

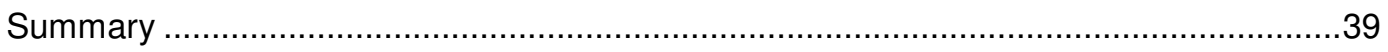

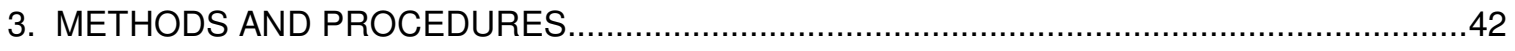

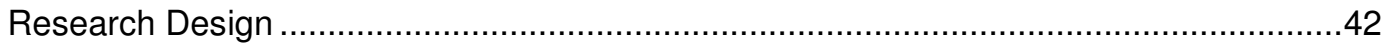

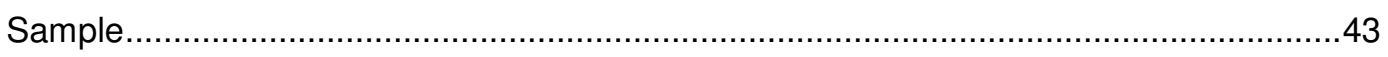

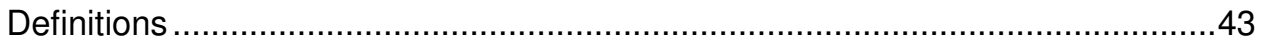

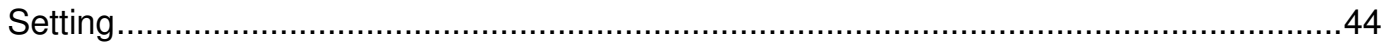

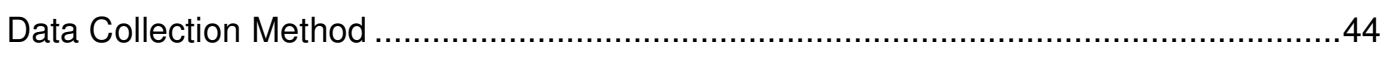

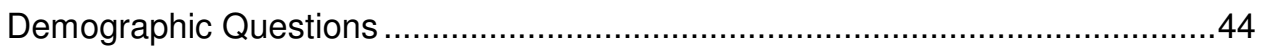

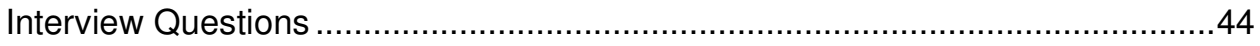

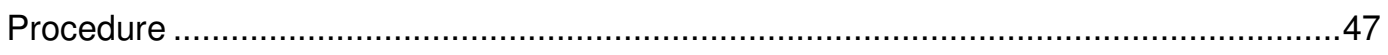

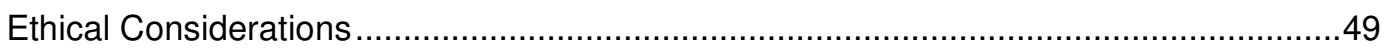

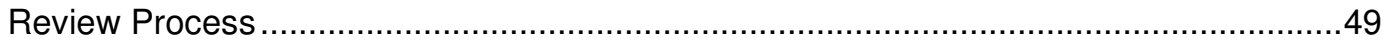

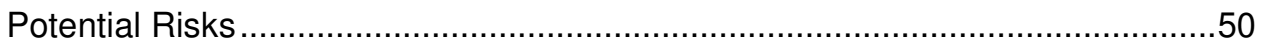

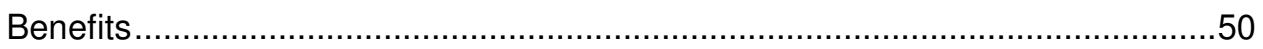

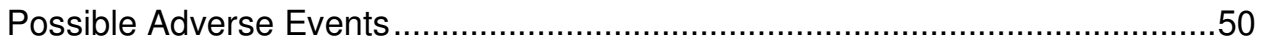

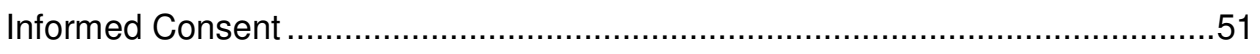

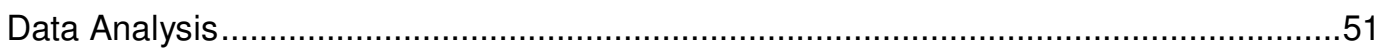

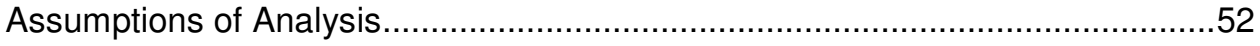

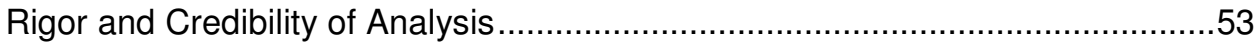

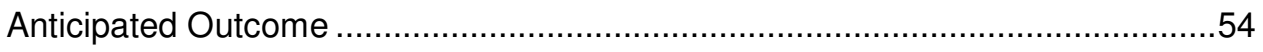

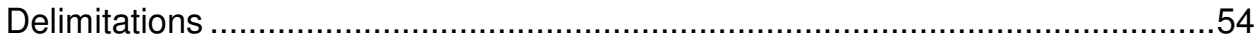

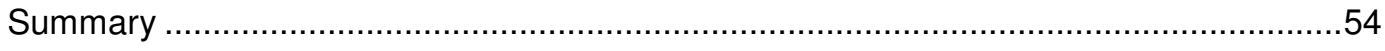

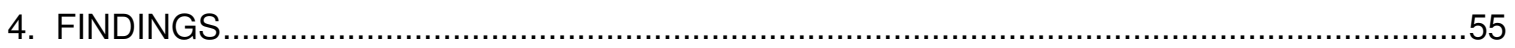




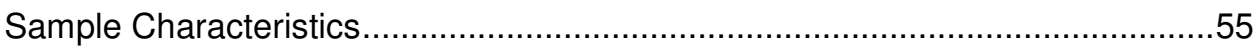

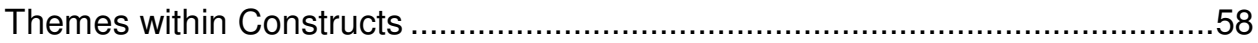

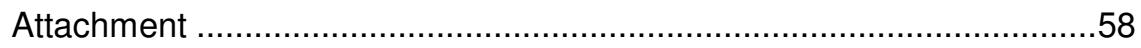

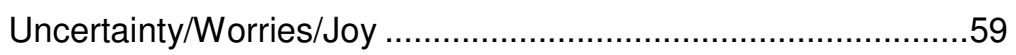

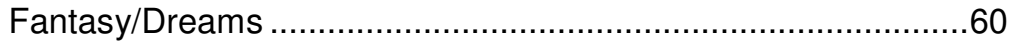

Unplanned Pregnancy/Acceptance .....................................62

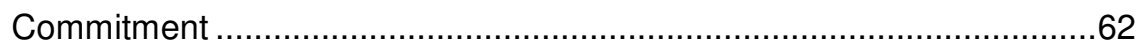

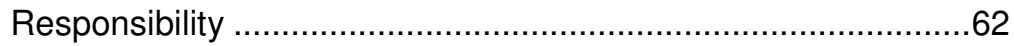

Sacrifices, Changes, Missing out …........................................63

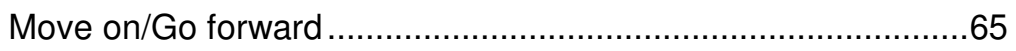

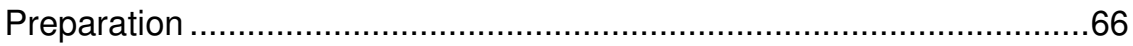

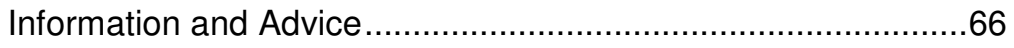

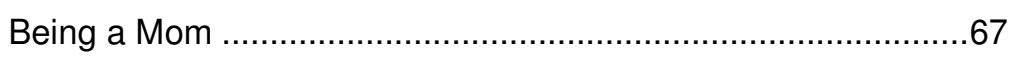

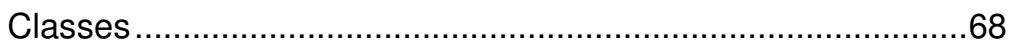

Culture, Family, Friends and Partner .................................................69

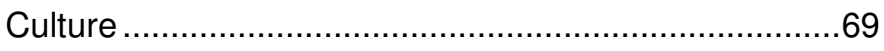

Family, Friends, and Partner ........................................70

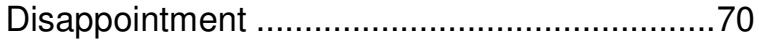

Support ................................................. 71

Advice ........................................................ 71

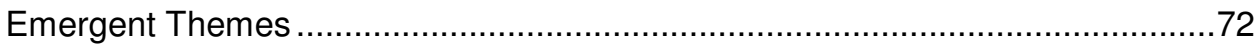

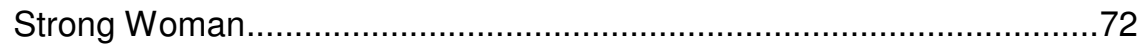

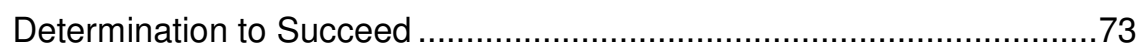

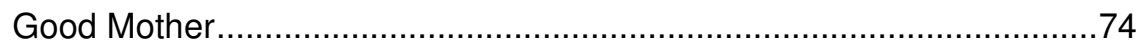

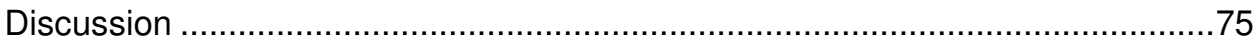

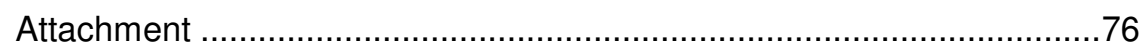




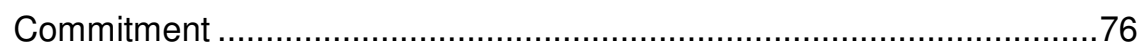

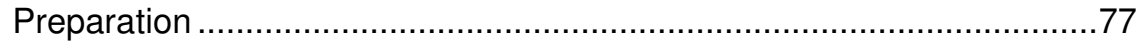

Culture, Family, Friends and Partner ...........................................78

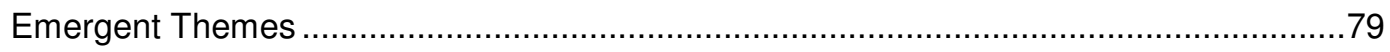

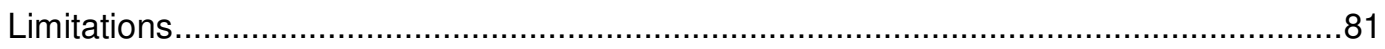

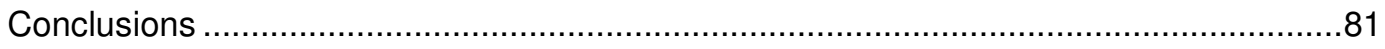

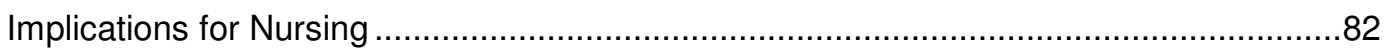

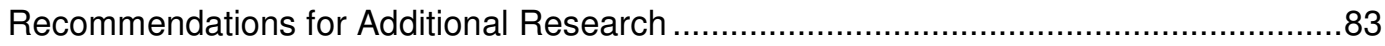

APPENDIX

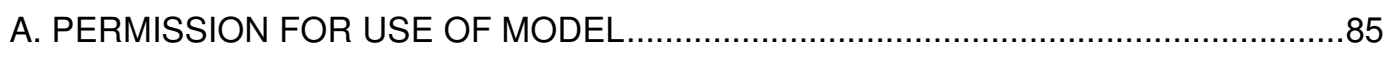

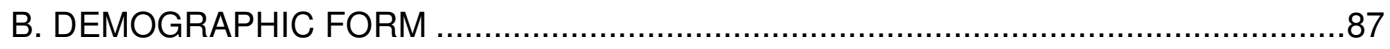

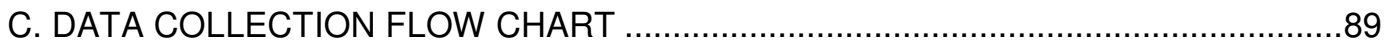

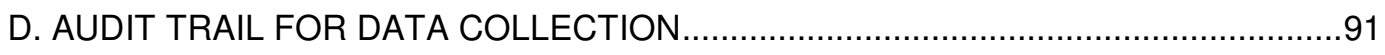

E. INSTITUTIONAL REVIEW BOARD CONSENTS, APPROVAL LETTERS .................94

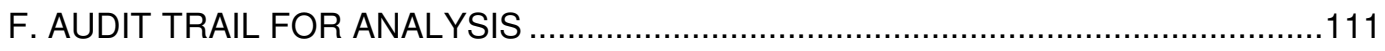

G. CHARACTERISTICS OF SUBJECTS INTERVIEWED .......................................113

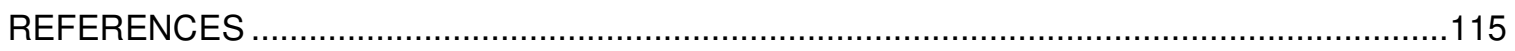

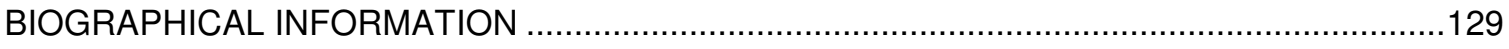




\section{LIST OF ILLUSTRATIONS}

Figure

Page

1. Conceptual model. Process of anticipating mothering-pregnancy

in the Mexican origin Woman .............................................................................. 


\section{LIST OF TABLES}

Table

Page

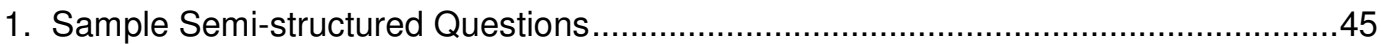

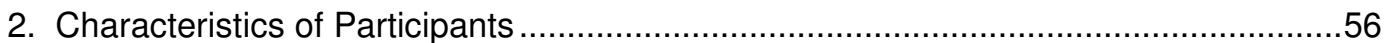




\section{CHAPTER 1}

\section{INTRODUCTION}

Having a baby is a significant life event for a woman. She faces increasingly complex situations and has few mothering role models. Mothering is an art and a science and is learned in interaction with a particular child (Mercer, 1995). In addition, mothers are expected to balance motherhood with multiple other roles. Over a woman's life span, the mother develops skills and abilities that she brings to the role of mother. Women do not experience pregnancy and motherhood in the same way (Sawyer, 1999). The model, "Anticipating Mothering in the Mexican Origin Woman", is presented in this chapter. The process of anticipating mothering for this study occurs during pregnancy and includes attachment, commitment, and preparation. Her culture and family, partner, friends influence the woman as the core of process of mothering. As the United States increases in diversity, there needs to be a greater understanding of how women of various ethnic groups experience pregnancy and motherhood so nurses provide health care that is beneficial and satisfying (Koniak-Griffin, Logsdon, Hines-Martin, \& Turner, 2006).

Hispanics in the United States comprise the largest and fastest growing minority group in the United States. The largest subgroup of the Hispanic population is of Mexican origin and is much younger than the United States population as a whole (U. S. Census, 2007). Information on how this group of women experiences pregnancy and anticipates becoming mothers will help guide practice and may provide implications for public policy. This information will help in planning programs to affect change and offer support to enhance the lives of the women and their family. The purpose of this chapter is to present the background and significance, the theoretical perspective, purpose, study questions, and assumptions for this study. 


\section{Background and Significance}

The Mexican American woman's age, biological make up, religion, culture, ethnicity, social organization, region of country in which she lives, and environment influence her as a unique cultural being. Female roles in Mexican American society are well delineated in the private sector of home and family where motherhood is highly valued. Although Mexican American women may work outside the home, this does not excuse them from their responsibilities at home. The Mexican culture emphasizes the needs of the extended family and there is a strong reciprocal relationship and loyalty to the family by the Mexican American woman. This relationship and the responsibility to her family has a huge impact on her relationship to others and the attainment of knowledge and use of resources within the community (Langaná \& Gonzalez-Rameriz, 2003). Guendelman, Malin, Herr-Harthorn, \& Vargas (2001) and Amaro (1988) suggested that the Mexican-American women's attitude to motherhood changed from being only in the home to being either a working or non-working mother. The women in these two studies showed more emphasis on individual and self-development, which is discussed further in Chapter 2.

Much of the research on mothering and the transition to motherhood has been conducted with White, middle-class women, and cross-cultural relevance has not been assessed (Sawyer, 1999). One study that has looked at the Mexican origin women was conducted by Domian (2001). The researcher questioned the experience social support has on Hispanic families during pregnancy in a northern New Mexico community. Four themes emerged from the interviews: (a) cultural preservation through shared lived experiences, (b) family perpetuation through generational bonding, (c) stability change through community sustenance, and (d) integration of health care beliefs through shared dialogue between one culture and another. The socialization process that helped the pregnant women and family members adapt to change and to support the pregnancy promoted positive pregnancy outcomes. This study provided a basis for future research to understand certain complex processes within the family and community. 
Following sixteen Mexican American women during the first 27 weeks of their pregnancy, Berry (1999) sought to understand the meanings and expressions of generic and professional care during pregnancy in their home and prenatal clinic. All of the women were born in Mexico and immigrated to the United States one to eighteen years' prior. Three to nine interviews with the women throughout the pregnancy revealed that generic culture care was essential and valued by the women to assure healthy childbearing. Six themes emerged from the interviews. The first theme, generic prenatal care was defined as the protection of the mother and fetus. Older Mexican American women transmitted this care intergenerationally over time influenced by religion and family beliefs and practices. Another theme of generic culture care was family obligation for providing for the family in times of need, sharing of self, and being with the childbearing mother. Culture care was respect for family caring roles based on age and gender. The Mexican American women viewed culture care by professional nurses for their knowledge, explaining and being attentive and for their protection. They also viewed culture care as the use of Spanish language in caring interactions. The women valued professional prenatal care where legal, economic, and technological factors influenced the social structure of the care. The women in the study were able to maintain both their generic culture care and professional prenatal care. The women delivered healthy full-term infants supporting the assumption that caring acts and processes are essential for fetal development and a healthy birth. The generic care beliefs and values were found as important aspects to provide culturally congruent care and nurses need to understand this cultural care knowledge (Berry, 1999).

Because the Mexican origin population in the United States is expected to continue to grow over the next 30 years (Berstein \& Edwards, 2008), it is important to conduct research with the young women of this population for two reasons. There is a need to understand what type of guidance they need during pregnancy to assist them to identify their competencies in order to plan evidenced-based culturally appropriate interventions. Second, there is need to understand 
their process of becoming a mother because of the effect mothers have on their health and the health of their child.

Becoming a mother requires extensive psychological, social, and physical work (Mercer, 2006). Deutsch (1945) and Benedek (1956) were among the first to describe the psychodynamic development of mothering and motherhood. According to Deutsch (1945) motherhood is the "relationship of the mother to her child as a sociologic, physiologic, and emotional whole" (p.17). This process begins with pregnancy and continues through birth, feeding, and care of the child. Motherliness is a character quality that is the woman's whole personality and an emotional phenomenon that seems to be tied to the helplessness of the infant (Deutsch, 1945). Benedek (1956) considered mothering a complex behavior pattern that is "innate and regulated by hormones" (p. 272). Development of motherliness is determined by many factors that surround the mother and on which she is dependent. The child is only one of these factors. Others are her social and economic life, her old and new experiences, her relationships to the baby's father and her family, and the position of the child in her existence (Deutsch, 1945).

Rubin $(1967 ; 1984)$ provides the knowledge base for researchers and clinicians to continue to define mothering and motherhood. Building on Rubin's work, Mercer (1995) concludes that mothering is an art and a science and is learned in interaction with a particular child. "Maternal behaviors include the blend of nurturing, caring, teaching, guiding, protecting, and loving that enhances the infant's physical, emotional, social, and cognitive development to adulthood" (Mercer, 1995, p. 1). The mother as a part of a particular culture influences and is influenced by that culture's values, customs, and rules of conduct as she "mothers" her child (Mercer, 1995).

Pregnancy can provide an important opportunity for psychological preparation for mothering and it can be either a disorganizing or a developmental opportunity. Little is known about how the sociocultural aspects of her world influence the mother's commitment and preparation for mothering. Only the mother can provide this information about her perceptions of 
self as a mother and how engagement with key others help in the process of becoming a mother (Smith, 1999).

\section{Philosophical/Theoretical Perspective}

The framework for this study was adapted from Mercer's theory, Becoming a Mother (Mercer, 2004; Mercer, 2006). Mercer's current framework is based on her original framework of Maternal Role Attainment (Mercer, 1981; Mercer, 1995; Mercer, 1986; Mercer \& Ferketich, 1990; Mercer \& Ferketich, 1995). Rubin's (1975) tasks of pregnancy and Thornton and Nordi's (1975) four stages of role acquisition provided the background for the original model that is discussed in greater detail in Chapter 2. Other qualitative and quantitative research (Nelson, 2003; Porter \& Hsu, 2003; Priel \& Besser, 2001; Siddiqui \& Hagglof, 2000; Sawyer, 1999) provided support of her original work. Walker, Crain, and Thompson (1986a; 1986b), Koniak-Griffin (1993) and McBride and Shore (2001) raised questions about the Maternal Role Attainment constructs that led Mercer to replace the framework with the present theory, Becoming a Mother (Mercer, 2004). Mercer denotes mothering as an ongoing process that never ends. It is a continuous process, ever evolving, that requires ongoing development. The stages leading to maternal role identity are: (a) commitment, attachment, and preparation (pregnancy); (b) acquaintance, learning, and physical restoration (first 2-6 weeks after birth); (c) approaching normalization (second week to 4 months after birth); and (d) integration of maternal identity (approximately 4 months after birth) (Mercer, 2004). The model, Becoming a Mother, suggests each stage as variable, fluid, and overlapping. Variables that influence the length of the stages are the mother, infant, family, and the environment. Mercer (2004) incorporated Bronfenbrenner's (1979) concept of interacting environments using circles to represent family and friends, community, and the society at large. The mother, father, and infant are interacting in the center within these living environments.

The first stage may begin even before pregnancy, when women begin to improve their health or family situation. After conception, a woman makes a commitment to the pregnancy 
and strives to ensure the health of the baby. She begins an attachment to the unborn baby that is heightened after she feels fetal movement. She daydreams about being a mother and seeks approval and support from family. Her preparation begins with the help of others such as her mother and family (Mercer, 2004).

The pregnancy stage has long-range implications for how well she moves through the other stages. An important element of pregnancy is the attachment experience with its unique elements. The woman first becomes attached to the idea of being pregnant and then gradually develops an attachment to the fetus (Rubin, 1984). Prenatal attachment may take as long as nine months as the woman restructures her life to care for her infant (Mercer, 1986).

Meaning to the mother's life is based on her attachment relationships (Müller, 1996). During pregnancy, women often pay attention to other attachments in their lives. The mother's relationship with the baby's father, her family, and the community evolve as she moves through the process of becoming a mother. The interactions between the mother, infant, and father are at the center of the interacting living environments (Mercer, 2004; 2006).

Although significant quantitative and qualitative research has been conducted to formulate Mercer's (2004) framework, there is a need for significant research with different cultural groups to understand what is necessary for the safety and health of the mother and her child (Koniak-Griffin et al., 2006). The focus of this study with the Mexican origin woman is the anticipatory stage (pregnancy).

\section{Conceptual Model}

The study's conceptual model provides the concepts to allow the Mexican origin pregnant woman's emic views of attachment, commitment, and preparation during pregnancy to emerge during an interview with the researcher. The model is an adaptation of Rubin's work on mothering and the pregnancy stage of Mercer's theoretical model, Becoming a Mother (Mercer, 2006). The adapted model is the anticipatory stage of mothering and emphasizes culture and the 
partner, family, and friends. Mercer's permission to use an adapted model of her work is in Appendix A.

Attachment begins with the woman becoming attached to the idea of being pregnant and slowly moves to attachment to the fetus (Rubin, 1984). As the mother gains knowledge and feels fetal movement, a unique affectionate relationship develops. This relationship leads to the future acceptance, nurturance, and protection of the child (Leifer, 1980; Rubin, 1984; Muller, 1990; Mercer, 2006).

Commitment involves making the decision to mother, giving up a concept of the self as she once knew herself, accepting the need to sacrifice, and accepting responsibility for the child. The mother strives to assure a healthy outcome by giving up unhealthy practices and seeking advice from family and health care providers (Nelson, 2003; Mercer, 2006).

Preparation for her new role involves physical and psychological preparation for pregnancy and the child. She envisions herself as a mother, contemplates her life as a woman with a child, and learns the expectations of becoming a mother. This preparation involves a woman's life experiences and may require psychological and social adjustments. She enlists the help of others in making physical and mental preparation for labor and the baby (Rubin, 1984).

Rubin's work and Mercer's first stage of Becoming a Mother served as basis for the theoretical framework for this study (Rubin, 1967, 1984 and Mercer, 2004, 2006). The model (Figure 1) Anticipating Mothering (Pregnancy) in the Mexican Origin Woman depicts the anticipatory stage of mothering for the woman. This model is adapted from Rubin's $(1967,1984)$ and Mercer's $(2004,2006)$ work on the woman becoming a mother. Mercer's anticipatory stage (pregnancy) was described as a time of initial adjustment, socially and psychologically (Mercer, $1981,1986,1995)$. The woman is an integral part of her environment. The environment includes family and friends, community, and society at large. Her partner, family, friends, and cultural influences interact with the woman during her pregnancy influencing the process of becoming a mother. Family and friends include social support, family values, cultural guidelines, parenting 
and family functioning, and stressors (Mercer, 2004). Culture is a powerful phenomenon and influences all aspects of life (Kreps, 1994).

The anticipatory phase of mothering may begin before conception with expectations often incomplete. For this study, anticipating mothering is a process that occurs during pregnancy, as the woman becomes a mother. This process is influenced by many factors in the woman's environment past and present. Each woman is unique and anticipates mothering in her own way but with similar occurring in all women.

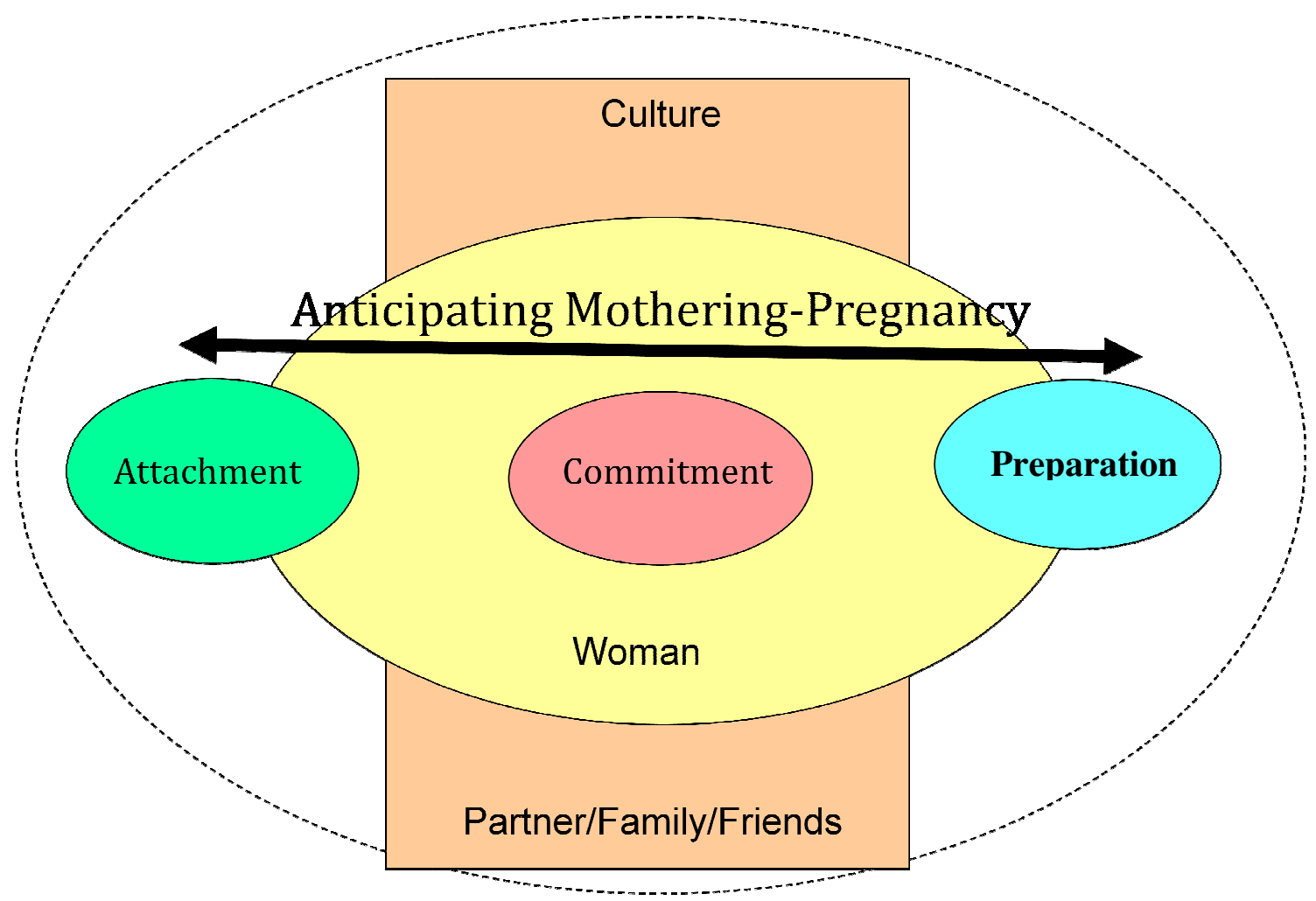

Figure 1. Conceptual model. Process of anticipating mothering-pregnancy in the Mexican origin Woman (Baxley, 2007). Adapted from Rubin and Mercer. Permission for adaption from Mercer.

Characteristics of culture that influence and shape the mother's views of mothering need further exploration. Kowledge of the concepts presented in the model and how they interact in the 
woman's environment will provide information about the meaning of anticipatory mothering for this group of women.

\section{Purpose}

The purpose of this study was to explore the meaning of attachment, commitment, and preparation during pregnancy as an anticipatory phase of mothering for the Mexican origin woman giving birth in the United States. A secondary goal is to provide beginning information about this group of women to assist them and their family in mothering.

\section{Study Questions}

The questions for this study are:

1. How do Mexican origin women describe attachment to their fetus?

2. How do Mexican origin women describe their feelings of commitment to the pregnancy and the idea of mothering?

3. How do Mexican origin women describe preparation in pregnancy for being a mother?

4. How do Mexican origin women describe the meaning of mothering during pregnancy?

5. What is the meaning of family to Mexican origin women during pregnancy?

6. What is the meaning of culture; the attitudes, beliefs, and taboos to Mexican origin women during pregnancy?

\section{Assumptions}

To reveal the meaning of the anticipatory stage of mothering in the Mexican origin woman assumptions were based on the investigator's professional experience as a perinatal nurse working with this population and on the literature review regarding mothering and culture. Assumptions for the study included:

1. Anticipating mothering in the Mexican origin woman is a process that begins during pregnancy. 
2. Anticipating mothering in the Mexican origin woman includes fetal attachment, commitment, and preparation.

3. Mexican origin women can describe feelings/emotions about pregnancy and becoming a mother.

4. Culture influences the anticipatory process of mothering for the Mexican origin woman.

5. Family and friends influence the process of anticipating mothering for the Mexican origin woman.

\section{Summary}

Much of the research that addresses the concept of mothering, especially that aspect of the process, which precedes birth, is based on studies of Caucasian middle class women. Studies are needed to understand the extent to which the results of studies (evidence) are useful to understand mothering in other growing populations such as Mexican origin United States residents. The theoretical framework for this study was adapted from Rubin's $(1964,1984)$ work on pregnancy tasks and Mercer's theory, Becoming a Mother (Mercer, 2004; Mercer, 2006).

Their work and the work of others using their theories provided the background for examining the concepts of attachment, commitment, and preparation among Mexican origin women. 


\section{CHAPTER 2}

\section{CRITICAL REVIEW OF RELEVANT LITERATURE}

Rubin's work guided much of the work on the concept of mothering and how it evolves during pregnancy. Rubin's framework is primarily pregnancy and the period following delivery. Others have modified or adapted her work. The Review of Literature provides the background for the study. It begins with a historical overview of mothering, and provides an in-depth description of the theoretical framework by Mercer $(1981,2004)$ on "Maternal Role Attainment" and "Becoming a Mother." The review continues with the psychosocial aspects of pregnancy, maternal-fetal attachment, commitment, preparation for pregnancy, and research using the maternal attachment theory (Cranley, 1981; Muller, 1992, 1993) . Lastly, the literature on the influence of the Mexican origin culture on the beginning process of mothering demonstrates the need for further research in this area.

Mothering from a Historical Perspective

Early mothering sources are the basis for the work undertaken by theorists who developed the framework for present day research. Rubin (1984) discussed the two beliefs held in the nineteenth century on the source of motherhood. The first is that maternal behavior is "instinctive". The force is set in motion genetically, is dormant for twenty more or less years, and then suddenly emerges epigenetically with the birth of the baby. The other theory holds that maternal behavior is formed early in childhood and is a copy of the mother's behavior. These behaviors begin and continue for all subsequent children. There is nothing preprogrammed or predetermined (Rubin, 1984). This later view is upon which much of the theoretical evolution is based.

Mead's (1934) and Sarbin's (1954) role theories considered role as a unit of socialization. The "self" is different from the physiological organism proper. The self is not present at birth, but 
arises and develops in the process of social experience and activity. Self develops in the individual as a result of her relationship to the process and to the other individuals in the process (Mead, 1934). Play, fantasy, empathy, and copying are mechanisms for "taking-in-the role-of other" and "adopting-the ways-of others" (Mead, 1934; Sarbin, 1954; Rubin, 1967b).

Deutsch (1945) and Benedek (1956) addressed mothering, motherhood, and motherliness from the psychodynamic developmental point of view. Deutsch (1945) suggested that the mental development of the mother is not linear and progresses as a spiral, affected by both biological and social development. Deutsch posited that motherhood is the relationship of the mother to her child (Deutsch, 1945). This process begins with the conception of the child and continues throughout pregnancy, birth, feeding, and care. The woman incorporates the fetus and invests it with narcissistic love until she gradually becomes aware of the fetus as a separate individual different from herself. Motherliness embodies two ideas: "a definite quality of character that stamps the woman's whole personality; and emotional phenomena that seem to be related to the child's helplessness and need for care" (Deutsch, 1945, p.17). Benedek (1956) suggested that mothering refers to the activities and practical aspects necessary to care for the offspring and guide its maturation and learning. Motherliness is the quality of the woman's personality that provides the emotional energy to maintain the tasks of mothering. Mothering is a complex behavior pattern that is "innate and regulated by hormones" but motherliness does not require childbearing alone but includes the infant and their behavior as well (Benedek, 1956, p. 272). Rubin (1984) suggestions that the maternal-child relationship as proposed by Benedek is important in that maternal behavior is not possible without the child. Each maternal child relationship is different.

Rubin (1984) suggested that it is more relevant to look for the origins of maternal behavior in the experience of the woman becoming a mother. She believed that the ideal theory should "explain the phenomenological and subjective experience of women becoming mothers in 
validatable terms and in a way sufficient to provide testable hypotheses for the variations in the human experience of mothering" (p.3).

\section{Early Studies}

The terms maternal identity and maternal role attainment were introduced by Rubin (1967a, 1967b) and later used by Mercer (1981) to describe the psychological processes that occur during pregnancy and postpartum. Rubin's field study of five primiparas and four multiparas women provided an in-depth look at their pregnancies through the first month postpartum. The primiparas were interviewed 12 times during the pregnancy starting as early as the twelfth week and 11 times the first month after delivery. The multiparas were interviewed an average of six times during pregnancy and five times post partum. Rubin hoped to determine processes involved in the acquiring of the maternal role. She wanted to identify who were the models or referents for maternal role expectations.

The interviews were analyzed and coded using a classification system devised by Rubin from preliminary and independent interviews and observations in maternal role attainment. Three dimensions of maternal role attainment were identified (a) the self-system or self-concepts as object, (b) cognitive maternal-role taking operations as process, and (c) models or referents as the subject (Rubin, 1967a, 1967b). The self-system categories elicited from the data included the ideal image, the self-image, and the body image. Expression of qualities, traits, attitudes, and achievements that the subjects found desirable for maternal behavior represented the ideal image. The representation of self-image was the consistent "myself" that has little or no historical self, but is an accumulation and continuation of self into the here and now (Rubin, 1967b).

Role theory does not usually consider body image; however, maternal role taking, generally is dependent upon body accommodations, functions, and capacity. Changes in the body are significant and loss of functional control lowers self-esteem and raises the risk of role failure (Rubin, 1967b). Body changes take on different meanings depending on when they occur in the pregnancy. Weight gain early in the pregnancy is welcomed but later the weight may seem 
ominous, 'Maybe it's a horse or an elephant, not a baby' (Rubin, 1967a, p. 240). Multigravidas were more likely to anticipate and deal with the body image of becoming a mother than were primigravidas.

Maternal role taking operations are a continuous process. The operations involved in the "becoming" of a mother fall into five categories: two forms of taking-on, two forms of taking-in, and one form of letting-go of a former status or role. Visualization of the entire process as a sphere with the core being role identity and with the outer layers being mimicry, role-play, fantasy, introjection-projection-rejection, and grief-work are presented as part of Rubin's initial work (Rubin, 1967a, 1967b).

Mimicry is the trying on of the role, "dressing the role". The women studied and laboriously imitated the expectation of the "how" to understand the role. Mimicry took on a magical property. Activity of mimicry is the "how" and expectation is the "what". Every pregnancy had the magical properties of mimicry even though the women may have considered it "silly". It may help the mother move on to the taking-in phase when the magic of mimicry was not effective and "let-her-down" (Rubin, 1967a, 1967b). Examples of mimicry were as simple as the wearing of maternity clothes even before necessary and avoiding lifting, falling, or eating particular foods (Rubin, 1967b).

Role-play is similar to mimicry but goes beyond the symbolic manifestations into the acting out of "what a person of this position does". Women search their environment for a subject to use for role-play (Rubin, 1967a, 1967b).

Fantasy and Introjection-Projection-Rejection (I.-P.-R.) are a part of the taking-in behavior and focus on "how it will be for me" and not the stereotypical ideas of "how one in this position behaves". Early in pregnancy, the fantasies focus on the self. Later, after feeling fetal movement, they focused on the child. The I. -P. -R. operation had the woman searching the environment and memory for models and precedents in role status, behavior, and events. The women searched these two roles for a "fit". If it was a good "fit" it served as reinforcement, and if 
an unsatisfactory "fit", it was rejected. This allowed them to add to their repertoire of knowledge and behaviors.

The operation of grief work is the letting-go of a former identity that is incompatible with the assumption of the new role. It is a review of memories of the attachments and events of another role. The details of the memories serve to loosen the ties to the former self without a child (Rubin, 1967a, 1967b).

Maternal identity was the end or goal in maternal role attainment when the "subjects had a sense of being in their roles, a sense of comfort about where they had been and where they were going" (Rubin 1967b, p. 243). Rubin assumed that the childbearing period is the preparatory period in maternal role acquisition.

The models for maternal role expectations all tended to be sex and situation specific for both primiparas and multiparas. The woman's mother was the most significant contributor to her anticipations in becoming a mother. Maternal role taking (childbearing, childbirth, childrearing) phases started with the woman's mother even if she was dead. If her mother was dead, she revived her in memory (Rubin, 1967a).

There was extensive use of peers as models. They were friends, neighbors, or acquaintances for primiparas or relatives for multiparas. Peers provided the evaluation of "what-Iam, who-I-will-be, and what-I-once-was of body image, ideal-image, and self image" (p. 343). The women saw the men as husbands and not as fathers. They were a source of support and reinforcement. Nurses became models for childcare after the child was born (Rubin, 1967a).

Rubin's (1984) later work described the phenomenon of role-taking in which the infant in utero was a partner in the mother's role-taking process. During the second trimester, after fetal movement, the mother's fantasies focused on the child. Gender, size, shape, and other characteristics were how the woman fantasized the ideal child. 'Binding-in' with the fetus brought about a bond between the mother and child and developed as the mother incorporated the child 
into her self-system. The formulation of maternal identity through the role-taking process and the binding-in and attachment to the child are interdependent coordinates of the same process (Rubin, 1984).

In Rubin's (1984) later work, she reformulated the maternal role attainment process into three operations: Replication, Fantasy, and Dedifferentiation. She deleted reference to "maternal role attainment" and preferred the term "maternal identity". The theoretical frameworks are generally consistent and based on her earlier works. With each pregnancy and each child, there is development of a maternal identity. It is a progressive series of cognitive operations manifested in conceptual and behavioral modes. The maternal identity formulation is gradual, systematic, and extensive. "The progression in cognitive operations and their respective conceptual and behavioral modes parallels the development of the pregnancy" (p. 39).

Replication includes previous role-play and mimicry. Using these, replication serves as a bridge to becoming a mother, a preliminary binding-in to maternal identity. Information from books, classes, and television provided unique or desirable elements to be incorporated through replication. Fantasy is the "projection in imagery of the mother and her child into the future: "how it will be'" (p. 44) and when she makes the child her own (Rubin, 1984). The third operation is an examination and evaluation for "goodness of fit" or identification of her current self-image. There is a trying-on, an introjection of a new element, and then a projection of herself with the new element. She then makes a decision to reject or accept the element as part of herself. Eventually in the neonatal period, the image stabilizes and she knows what to expect of the child. It is now "the way I do it" with security and confidence in knowing herself and the child as complementary individuals (Rubin, 1984).

Aspects significant in the development of the maternal role noted by Rubin (1984) and later by Lederman $(1984,1996)$ were the role of fantasy, the ability to resolve conflicts, the relationship between the expectant mother and her mother, the importance of role models, and the ability to form an attachment to the fetus. Maternal tasks the woman undertakes in 
childbearing are twofold: "to conserve the intactness of the self and of the family as ongoing, open systems and to orchestrate the assimilation and accommodations of this child into the self and family systems" (Rubin, 1984, p. 53). The child is only an idea during pregnancy and the child's identity is ascertained only after childbirth. The pregnant woman maintains her sense of self and the family systems while she assimilates and accommodates the child into the other systems. Four processes have been identified: the seeking of safe passage through the childbearing and childbirth, the binding in or seeking of acceptance of her child by significant members of the family, and conversely a seeking of acceptance, involvement, and commitment of self as the mother of her child. Lastly, there is a searching and exploring of the meaning of giving of oneself on behalf of another. Maternal tasks address three permeating systems: the "self system, the maternal-child subsystem, and the larger family system" (p. 54).

\section{Development of Mercer's Original Theory}

Mercer (1985) defined maternal role attainment "as a process wherein the mother achieves competence and comfort in the role and integrates maternal behaviors into her established role set” (p. 198). Using Rubin's work, which focused largely on pregnancy, Mercer $(1980,1981,1985, \& 1986)$ began a series of studies on mothers and maternal role attainment. She utilized Thornton and Nardi's (1975) stages of role development for her studies. The four stages are anticipatory, formal, informal, and personal identity. Mercer (1985), like Rubin, discussed the importance of the self-system and the infant in maternal role attainment.

Mercer's original model, Maternal Role Attainment, included stages in the process of role identity. The anticipatory stage (pregnancy) is a time of initial adjustment, socially and psychologically. Seeking information from those in the role and visualizing oneself in the role assist in learning expectations of the role. This is congruent with Rubin's (1984) operations of replication and fantasy (Mercer, 1981, 1986, 1995).

The formal role-taking stage begins with birth of the child when professionals and others largely direct this stage in the mother's social system. The informal role-making stage includes 
how the woman structures the role to fit herself according to her experiences and her goals for the future. She learns her infant's cues and begins to develop her style of dealing with the role. These behaviors are the dedifferentiation, the examination, and evaluation for a good fit with the current self-image, as described by Rubin and occur just before maternal identity. Reaching the last stage of personal role identity occurs when the mother has integrated the role into her selfsystem and is secure and competent in her identity as a mother (Mercer, 1995).

Mercer's original model placed the mother and infant within the family context to illustrate the complexity of the process. The father or intimate other helps to diffuse the tension in the development of the mother-infant dyad. The mother and infant affect each other as the maternal identity development moves through the stages and the child reaches new development stages (Mercer, 1986, 1995).

Mercer used Bronfenbrenner's (1979) nested circles of the microsystem, mesosystem, and macrosystem. The microsystem is the immediate environment and most influential on the maternal role attainment. The mesosystem "encompasses, influences, and interacts with persons in the microsystem". The macrosystem refers to the prototypes existing in a particular culture or transmitted cultural consistencies; the social, political, and cultural influences on the other two systems (Bronfenbrenner, 1979; Mercer, 1986, 1995).

Walker, Crain, and Thompson (1986a,1986b) suggested a reconstruction of the theoretical structure of maternal role attainment through their proposal that maternal role attainment serves as an organizational term for setting the boundaries of mothers' early experiences in parenting. This phenomenon appears to contain several distinct components: "maternal identity, perceived (subjective) role attainment, and demonstrated (behavioral) role attainment" (Walker et al., 1986b, p. 353).

Walker, Crain, and Thompson (1986a) agreed with Rubin (1967a, 1967b) and Mercer (1981) that the process of maternal role attainment is a universal process. Walker, et al (1986a) studied multiparas and primiparas during postpartum and found that although maternal identity 
and perceived maternal role attainment were convergent among primiparas, it was not true of multiparas (Walker, 1986b). They also found that maternal age, maternal socio-economic status (SES), and maternal education were significantly related to identity or role attainment. Supporting the work of Rubin (1984) and Mercer and Ferketich (1994), Walker found that primiparas formation of the relationship with their infants and gaining confidence in the parenting role appeared to be interdependent. Multiparas confidence did not seem to be related to their relationship with their infants (Walker et al., 1986b).

Mercer's Revised Theory, Becoming a Mother

Questions by Walker, Crain, and Thompson (1986a) and Koniak-Griffin (1993) about the behavioral and cognitive constructs of Maternal Role Attainment (MRA), and the suggestions by McBride and Shore (2001) on retiring the term MRA, led Mercer (2004) to replace the term with Becoming a Mother. The model, as described in Chapter 1, parallels the original stages in Mercer's theory, but embraces the experiences of the mother.

Maternal identity, the last stage of MRA, does not include the continued expansion of the self as a mother. Becoming a mother is larger than a role. It is a life-long process and connotes continued growth (Mercer, 2004). The new names for stages of the process were derived from the qualitative data described earlier and lead to maternal role identity: (a) pregnancy includes commitment, preparation, and attachment; (b) includes acquaintance, learning, and physical restoration first two-six weeks following birth; (c) moving toward a new normal during two to four months; and (d) achievement of the maternal identity occurring around 4 months (Mercer, 2004).

The concept of interacting nested ecological environments from Bronfenbrenner (1979) are still present in this revised model but are renamed to reflect the living environments of (a) family and friends, (b) community, and (c) society at large. The mother, infant, and father are at the center of the interacting environments. The environment of family and friends include social support, family values, cultural guidelines, parenting and family functioning, and stressors. Day care, places of worship, schools, work settings, hospitals, recreational facilities, and cultural 
centers are part of the community environment. The society at large entails a much bigger scope that is influenced by laws affecting woman and children, evolving reproductive and neonatal science, transmitted cultural consistencies, and national health care programs (Mercer, 2004).

Psychosocial Pregnancy: Commitment, Attachment, and Preparation Mead and Newton (1967) suggested that through the ages, society practices focus around childbearing to assure material and emotional support to the pregnant woman. Other societies provide more economic and practical help to childbearing women and infants than modern American society. Investigators seek to understand a mother's work during pregnancy and the influence this stage has on the other stages of becoming a mother.

\section{Empirical Studies}

Colman and Colman's (1973) research on pregnancy provided information on the psychological aspect of pregnancy. "Pregnancy is neither a static nor a brief experience, but one full of growth, change, and enrichment" (Colman \& Colman, 1973, p. 1). They suggest that it is rewarding and challenging because it is a new life for parents as well as the child. Three psychological stages of pregnancy emerged from their research: (a) incorporation, accepting the fetus as part of her body; (b) differentiation, realizing it is there but it is not a part of her; and (c) separation, preparing to give up the fetus. The risk for pregnant couples is that they do not grow through the process and participate in the rewards. Women benefit from being with other pregnant women for discussion of like problems and concerns. These groups can provide a critical social support when there is little family or community involvement (Colman \& Colman, 1973). The authors suggested a closer look at the psychological experiences that accompany pregnancy, motherhood, and parenthood (Colman \& Colman, 1973).

Pregnancy is a life crisis in the anthropological sense and the psychological sense. It is a transitional time when the individual is poised between the childless life and parenthood. Pregnancy is a process that must be experienced fully and is a regeneration of the human race and the individual, through confrontation with self, family, and culture (Colman \& Colman, 1973). 
The experience of pregnancy is a psychological event of great power and potential. It may soon be forgotten and is dependent on the birth of the baby for meaning; it should also be thought of as a critical developmental stage in its own right (Colman \& Colman, 1973).

Oakley (1979) reports women's response to first childbirth, the relation to other major life events, using the words of the women she interviewed. Childbirth, a biological act, is never just one event but is defined by the different cultures that women have invented as ways of living (Oakley, 1979). She suggested that the institution of motherhood is the way women become mothers in society today and reflects what has already happened to them, as they became women (Oakley, 1979). One of the women interviewed suggested that the questions related to sexual, personal, and physical aspects that Oakley asked during the interview process were important and missing from the health care they received. They thought their feelings about the experience should "get out" and accomplished in groups similar to rape counseling (Oakley, 1979).

Shereshesky and Yarrow (1973) delineated four variables needed to understand the psychological aspects of pregnancy. These include life history, personality of the woman, current life situation, and the pregnancy experience. Life history is important because of the woman's prior relationship with her mother on the development of the maternal identity and to adaptation to pregnancy and her future role as a parent.

Ways of coping, defenses, ways of expressing feelings, qualities of feminine identity, and achievement of the adult role help to determine how pregnancy is associated with personality changes. The husband's impact on adaptation to the maternity cycle and the marital adaptation are some of the current life situations that affect the woman's attitude toward the pregnancy and the mothering role. The psychological reactions to the physiological aspects provide the pregnancy experience (Shereshesky \& Yarrow, 1973; Sank, 1991).

The woman's personality characteristics of ego strength and nurturance and the confidence in visualizing herself as a mother and the physiological aspects of the pregnancy were 
considered predictors of pregnancy adaptation. Moods, expectations, hopes, concerns, and fears relate to the mother and her unborn child. Using anticipatory guidance may help by decreasing anxiety for the woman and man and allow them to respond to the dynamics of change associated with childbirth and the infant (Shereshesky \& Yarrow, 1973; Sank, 1991).

Extensive research on the relationship between psychosocial conflicts during pregnancy and complications that arise during labor, Lederman (1996) identified seven dimensions of maternal development. She noted pregnancy as a series of adaptive responses as the woman moved toward orientation to the maternal role. Pregnancy is seen as the transition or paradigm shift between two lifestyles, "the woman-without-child" to a "woman-with-child" (p. 19). The normal course of childbearing is a type of test that is a part of the mother's growth rather than a crisis. When the two lifestyles are far apart, there is a sense of crisis. During the nine month period of pregnancy, there is a reevaluation of the self and significant others. It is the discarding of old ways and taking on of new ways. Pregnancy is a period of preparation where the baby is an important part (Lederman, 1996). The seven dimensions of maternal development include: (a) the acceptance of and adaptation to the pregnancy; (b) a progressive development in formulating a parental role and a relationship with the coming child; (c) the impact of the pregnant woman's relationship with her husband on pregnancy adaptation and vice versa; (d) the woman's relationship with her mother; (e) knowledge about the reasonable preparation for the events of labor; (f) the anticipation of mechanisms for coping with fears concerning pain and loss of control in labor; and (g) coping with fears involving loss of self-esteem in labor (Lederman, 1996).

Miller (2005) followed seventeen women through the year that they became mothers using a narrative approach. The researcher wanted to capture the woman's accounts of her experiences and not the woman's public accounts influenced by the medical profession. The women were interviewed on three different occasions; the first at eight to nine months pregnant; the second at six to eight weeks postnatally and; third was at eight to nine months postnatally. Telephone contacts provided additional information, and an end-of-study questionnaire collected 
information on the women's experience of being a part of research. All of the participants were white, professional, and middle class with a mean age of 30 years at the initial interview, but still showed diverse and complex experiences. Miller suggests that becoming a mother involves physical and emotional experiences that generally result in the birth of a child and a sense of responsibility and feeling for the baby. Women now have little or no first-hand experience of pregnancy and babies. Their expectations generally do not match the real experiences of motherhood. When asked to describe being pregnant, the participants attempted to make sense of this period of transition. Demonstration of their attempt was in the following areas: preparation and engagement with experts (preparing appropriately and anticipating the birth), shifting the sense of selves, and anticipating motherhood (Miller, 2005). According to Miller (2005), preparing appropriately, in a moral context, was hard to escape. Some felt the influences even before conception. To anticipate and prepare to become a mother was appropriate to society's expectations. To engage in early health care and have their pregnancy monitored were the expectations of their group of family and friends. The participants wanted the experts to monitor the pregnancy and wanted more information, not less. Although the participants said they had no expectations of what pregnancy would be like, the narratives showed the message, "I'm just doing what you're meant to do" (Miller, 2005, p.72).

When anticipating the birth, the participants used words such as natural, naturally and instincts within the context of hoping for a 'good' birth and accepting what medical professionals had to offer. They felt that 'good' mothers achieve a safe birth without pain relief. The awareness of how women achieve birth provided perceptions of the type of mother they would be. A 'good' mother gives birth 'properly', or a 'guilty' mother is one who fails and needs medical interventions (Miller, 2005).

A shifting of self was the transition to motherhood experienced as physical changes and changing perceptions. The mother experienced how she saw herself in the eyes of others as a private and personal transition regulated and monitored by the public. This personal transition 
required self-surveillance and personal policing. These potential struggles are perceived in relation to the lack of control and changes in her body. The ideologies of mothering are dominant and powerful and remained constant in shaping both engagement and resistance in the transition (Miller, 2005).

Anticipating being a mother involved uncertainty. The women used words such as 'worried' and 'frightened.' The context of the anticipatory narratives constructed the ideas of mothering as 'natural.' The women were concerned with getting it right. Their concerns had implications for both their sense of self and the ways in which others perceive them. There was the struggle between trying to retain a sense of self while negotiating the expectations of them culturally as a woman in transition to motherhood (Miller, 2005).

The public expectations and assumptions and the woman's private experiences did not always coincide in their journey to motherhood. The women wanted others to see them as preparing appropriately to become a mother but felt the uncertainty that this period of transition brings. The characteristic of this period of transition included active engagement with experts that showed them as acting responsibly and avoiding unnecessary risks. Being responsible also involved some level of self-governance. The women rarely voiced their personal journey into motherhood in the antenatal period but presented an acceptable preparation for motherhood based on what they thought others wanted them to do (Miller, 2005). 


\section{Maternal/Pregnancy Theories}

Leifer (1980) made the theoretical assumption that pregnancy and early parenthood are a series of developmental tasks and represent a unique stress or conflict that must be resolved for maturation to occur. Studying 19 white middle-class primigravidas, Leifer attempted to understand some of the psychological changes that occurred during the first pregnancy and early postpartum months. Although emotional upheaval and rapid change associated with pregnancy were present, some women showed a growing sense of adulthood and integration of a new maturational stage that co-existed with emotional disequilibrium. The degree of personality integration achieved by early pregnancy predicted the extent of psychological growth experienced throughout pregnancy and early parenthood.

Sherwen (1987) noted a lack of content in nursing concerning psychosocial aspects of individuals and families during pregnancy. She introduced the concept that the entire family shares in and is affected by woman's pregnancy. Her thinking was that it is not just a pregnant woman with a family, but a family who shared the pregnancy experience in a dynamic manner. As others have suggested, Sherwen suggested crisis as a model for nursing intervention with the pregnant family.

Like the woman, the family has to achieve developmental tasks during pregnancy as well. They must first accomplish the task of establishing themselves as a family. Other tasks that are necessary are: reevaluating responsibility, adapting sexual relations, adapting extended family relations, maintaining family members' morale; and preparing economically, physically and emotionally for the new member. The theme of the family is pregnancy, and it affects all members. They must all adjust and adapt (Sherwen, 1987).

Maternal-fetal Attachment

Leifer (1980) and Rubin (1984) stressed the importance of the woman's interaction with her fetus. They suggested that a process occurred where the woman developed knowledge and 
feelings for her unborn child. Rubin (1984) suggested that pregnancy was the first phase of the binding-in process that continues after birth and is the basis for the mother's relationship with her child.

Over the past 25 years, varied studies have been conducted in relation to maternal-fetal attachment. Cranley (1981) created the maternal-fetal attachment (MFA) construct defining it as "the extent to which women engage in behaviors that represent an affiliation and interaction with their unborn child" (p. 282). She created and operationalized the construct with the maternal-fetal attachment scale (MFAS).

Cannella (2005) noted mixed outcomes from an integrative literature review of research on maternal-fetal attachment. Looking at correlational studies of psychosocial variables in relation to MFA, Cannella noted that the studies yielded disappointing findings. Sixty findings about MFA showed that 23 were statistically significant and 37 were not. Creation of a strong knowledge base for MFA has not occurred. Demographic variables, such as age, education, income/socioeconomic status, marital status, and race/ethnicity have relatively unaffected the results of MFA in 24 of the 38 findings reviewed. Younger and first time mothers were found to have higher MFA scores (Mercer, 1995; Pascoe \& Kototailo, 1995; Lindgren, 2001). One area that suggests that MFA is a useful construct to study in relation to some of the demographic variables is that of attitudes toward childbearing. Of the fifteen findings in the analysis of pregnancy-related variables, eight were statistically significant, while seven were not. Cannella suggests that researchers need to continue to explore these relationships to add to the knowledge base about MFA. Findings in longitudinal studies consistently showed changes towards a stronger MFA as pregnancy progressed and some predicted outcomes in the postpartum period. Of the 25 finds in Canellas's analysis looking for psychosocial variables such as social support in relation to MFA, 20 were statistically significant and five were not. Cannella suggested there is a need for more correlational studies to better understand what factors contribute to MFA. Comparative studies are needed to understand differences in MFA between 
high- and low-risk pregnant women. The correlational studies could then lead to experimental research to test predictive theories. The MFA has important implications for pregnant women worldwide, but studies reviewed by Cannella (2005) found the majority of the studies were conducted in the United States. Cultural differences and ethnic perspectives need to be determined.

Müller (1990) defined prenatal attachment as "the unique, affectionate relationship that develops between a woman and her fetus" (p.11). This definition, her extensive review of the literature related to maternal-fetal attachment, and Cranley's MFAS provided a means for development of the Prenatal Attachment Inventory (PAI). She developed the PAI because she felt the MFAS did not emphasize affiliation but behaviors of antepartal tasks of maternal role attainment. She felt the use of two measures would enhance the interpretation of the data that are contradictory and inconsistent in current prenatal attachment research. Correlating PAI scores with scores from the MFAS scores tested the PAI construct validity. Factor analysis of the PAI and the MFAS did not support prenatal attachment as a multidimensional construct, but suggested that prenatal attachment may consist of one dimension. The study provided evidence for validity and reliability of the inventory. Müller suggested that the PAI and the MFAS used together could contribute to the validity of future studies (Müller, 1993).

Müller (1990,1992, 1993, \& 1996) suggested that evidence to support the activities in the literature are necessary before promoting prenatal attachment interventions or changing maternal expectations She suggested that future studies of pregnant women should include all ages, ethnic groups, and all stages of pregnancy to determine the normal range of scores for prenatal attachment and related variables.

Müller (1992) suggested that longitudinal studies are necessary to confirm the natural progression of prenatal attachment and its affect on the birth experience and postnatal attachment. This topic is of great interest to researchers, clinicians, and the lay public. When 
discussing this topic, interventions should improve the maternal-infant relationship and not cause additional stress to the mother by blaming women for children's negative traits (Müller, 1992).

Several studies used the PAI to conduct research on maternal-infant attachment (Müller, 1996; Siddiqui, Hagglof, \& Eismann, 1999; Siddiqui \& Hagglof, 2000). Müller's study compared prenatal and postnatal attachment in 201 women in Ohio. The majority, $94.5 \%$ were Caucasian with $3 \%$ black women and $2.5 \%$ Asian. They found a small correlation between prenatal and postnatal attachment. It was felt that other factors also influenced postnatal scores suggesting that caution is necessary when promoting increased prenatal attachment in hopes of improving postnatal attachment (Müller, 1996).

Siddiqui, Hagglof and Eisemann (1999) studied 171 Swedish women during the third trimester of the pregnancy using three questionnaires; the PAI, a questionnaire regarding psychosomatic symptoms, and one on attitudes towards pregnancy. Prenatal attachment as a multidimensional construct included factors such as fantasy, interaction, affection, differentiation of self from fetus, and sharing with others.

Culture

Although the previous studies have increased the body of knowledge related to mothering and psychosocial aspects of pregnancy, seldom are cultural considerations addressed.

Culture is rarely explicitly mentioned in the majority of studies. Failure to consider this can be taken to mean that either culture is irrelevant or that everyone believes and behaves in accordance with the dominant Anglo culture, which tends to be viewed as the norm (Cummins \& Scrimshaw, 1988, p.165).

The United States is a mosaic of many cultures and reflects a mixture of ideologies, beliefs, and health-care practices. As society becomes more diverse, health care professionals need to become aware of cultural and ethnic differences (Purnell, 2008).

Culture is a complex, multifaceted and powerful phenomenon influencing all aspects of modern life (Kreps, 1994). The study of culture arose from anthropology and sociology. In 1871, 
Taylor defined culture as an extension of biological capabilities, an interlinked web of symbols, and a device for channeling and limiting human choices. He further defined it as the medium of personhood and social relationships. Only part of culture is conscious and must be in two places at once, in the people's minds and in the environment as an act or artifact. Later, the author added to the definition; culture is learned and distinguishes man from other animals. Culture is the non physically inherited traits we possess and what we can tell, convey, or hand down to the next generation (Bohannan, 1992). Primary and secondary characteristics of culture are the influences that shape people's view and degree to which they identify with their culture. Primary characteristics are "nationality, race, color, gender, age, and religious affiliation" (Purnell, 2008, p. 7). Educational and socioeconomic status, occupation, military experience, political beliefs, urban versus rural residence, enclave identity, marital status, parental status, physical characteristics, sexual orientation, gender issues, reason for migration and length of time away from the country of origin are all secondary characteristics (Purnell, 2008).

Not only is culture important to understand when studying groups, but acculturation is an important construct to take into consideration. Acculturation is the exchange that takes place when individuals of two different cultures interact and have continuous contact. The changes that are observed take place across a number of different domains such as attitudes, values, behaviors, and a sense of cultural identity (Redfield, Linton, \& Herskovits, 1936; Cuéllar, Arnold, \& Maldonado, 1995; Ryder, Alden, \& Paulhus, 2000). The Social Science Research Council (Social Science Research Council, 1954) stated that acculturation is initiated by the conjunction of two or more autonomous cultural systems.

The Office of Minority Health operationally defines culture and provides a way to study cultures. Culture is "the thoughts, communications, actions, customs, beliefs, values, and institutions of racial, ethnic, religious, or social groups" (Office of Minority Health, 2001). 
This definition incorporates many of the central themes of the other definitions, and it is necessary for the researcher to understand these themes when conducting culturally linguistically appropriate studies.

Acculturation viewed as a group or individual phenomenon makes it difficult to specify the different domains (e.g. values, attitudes, interpersonal relationships, language, and behaviors) affected by this cultural process. Acculturation has a dualistic effect. It affects the culture of a group as well as the psychology of an individual, a person influenced directly by the external culture and by the changing culture of which the individual is a member (Berry, 2002).

Berry (2002) approaches his framework of acculturation with four strategies based on the quadrants defined by the two dimensions, bidimensional (and unidirectional) or multidimensional (with complex variations taking place). A bidimensional or multidimensional perspective of acculturation is a process in which the heritage and mainstream cultural identities are free to vary independently. Four acculturation strategies are based on the two issues of maintenance of ones on heritage culture and identity and relationships sought among groups. Acculturation stategies are based on two issues; ethnocultural issues (integration, assimilation, separation, and marginalization) and strategies of the larger society (multiculturalism, melting pot, segregagation, and exclusion). These are the ways in which acculturation can take place. Berry suggests that researchers need to learn about culturally rooted individual preferences and differences instead of assuming what these individuals want.

During the last half century, research about cultures has been conducted in the nursing discipline (Andrews, 2003). Madeline Leininger (1976) formally introduced culture awareness to nursing practice. She defined culture are "the learned, shared, and transmitted values, beliefs, norms, and lifeways of a particular group that guides their thinking, decisions, and actions in patterned ways" (Leininger, 1995, p. 60). Culture Care Diversity and Universality was the first established cultural nursing theory and used to discover culture care phenomena to provide culturally congruent care (Cohen, 1992). 
Other scholars continued to attempt to understand and redefine the study of cultures. The Purnell Model for Cultural Competency is a holographic and complexity theory. General knowledge of cultural aspects provides a framework with which health-care providers can assess multicultural populations. Using specific cultural knowledge one can maximize therapeutic interventions by becoming a co-participant and client advocate in diverse health-care settings. The health-care provider needs to understand clients' perspectives to develop an open style of communication, learn from the multicultural clients, and demonstrate tolerance for ambiguities inherent in cultural norms, which are ever evolving and changing (Purnell, 2008).

The Immigrant women: A framework for their health care, was developed by Alaf Meleis (2003) to provide quality care for women. This framework reflects the capacity of women to define and be defined by their interactions with their environment. To understand women's health care experience and responses, several components in this model are important: diversity, previous models of care, and development-all interacting to affect the process of care. The biomedical model, the reproductive and maternal model, and the cultural model join together to form her model.

Giger and Davidhizar (1999) identified six cultural expressions that make up the unique cultural identity. These are environmental control, biological variations, social organization, communication, space, and time orientation. Rachel Spector (2004) illustrated this assessment model to form the personal health traditions of a unique cultural being. Environmental control is the ability of cultural groups to plan activities that control or direct environmental factors. The phenomenon plays important roles in the way patients respond to health related experiences, including how they define health. Examples of biological variations are body build and structure, skin color, enzymatic and genetic variations, susceptibility to disease, and nutritional variations. The social organization in which people grow up in is essential to their cultural development andidentification. Children learn responses from their family and its ethnoreligious group. Differences in communication are presented in many ways, including language difference, verbal 
and nonverbal behaviors, and silence. Personal space is people's behaviors and attitudes toward the space around themselves. Individuals and ethnic groups use personal space differently. How time is viewed in the present, past, or future varies among different cultural groups and may influence how an individual seeks health care (Spector, 2004). Giger and Davidhizar's (1999) assessment model and Spector's (2004) depiction of the culturally unique being are useful across disciplines to understand cultural diversity and to learn techniques of culturally competent care.

Hispanics in the United States

The term Hispanic is the term used by government institutions. It refers to those individuals who reside in the United States and were born in or trace the background of their families to one of the Spanish-speaking Latin American nations or to Spain (Marin \& Marin, 1991). Hispanics comprise the largest minority and fastest growing group in the United States at 45.5 million, $15 \%$ of the total population (Bernstein, 2008). The Hispanic population is composed of individuals from many different areas and origins. The largest group is Mexican origin with the others representing at least twenty other national origin groups. The groups share much in common, but each is a distinct group (U. S. Census Bureau, 2007; Pew Hispanic Center, 2006).

The population of Hispanics in 2008 is much younger with the median age of 41 years, as compared to the population as a whole at 47 years. Native-born Hispanic women are even younger with a median age of 39 . Hispanic women comprise $48 \%$ of the Hispanic adults. The percentage of childbearing Hispanic women in the United States has increased as well. The fertility rate for Hispanic women is 84 per 1000 compared to 63 per 1000 Non-Hispanic women. Immigrant women account for the greater number with a fertility rate of 96 per 1000 women (Pew Hispanic Center, 2008).

The U. S. Census Bureau projects that by the year 2050, the Hispanic population will triple and represent $29 \%$ of the United States population. Most of the increase will be from United States-born Hispanics (Bernstein \& Edwards, 2008). Immigration was what promoted the growth in population in earlier years, but their children will drive the growth in the future. 
Hispanic Cultural Values/Family, Pregnancy, and Mothering

As the growth of the Hispanic population continues, it points to the need to consider cultural aspects of pregnancy, mothering, and the parenting experiences. Cultural context defines the norms that influence knowledge, attitudes, beliefs, expectations, and perceptions about this experience (Cummins \& Scrimshaw, 1988). The Latino culture is rich in folklore, beliefs, and traditions. The people are as diverse as their country of origin, but have a common ancestry thread and a native language, Spanish. Certain cultural values that affect the process of pregnancy and mothering need to be considered when studying this population (Marin \& Marin, 1991).

Diversity in this population is evident in levels of acculturation and assimilation, socioeconomic status, living conditions, and migration status (Mattson, 2003). With people of Mexican descent being the largest population of Hispanics, the cultural aspect of the lifestyles of these women is of particular interest. Mexican Americans view pregnancy as natural and desirable so many women do not seek prenatal evaluations or seek them late in the pregnancy. Many women seek family advice before seeking medical care. By incorporating cultural brokers that are known to the family may help to reduce conflict and empower them to seek health education and care (Zoucha \& Zamarripa, 2008). Incorporating folk practitioners, trained lay personnel from the community, the concept of personalismo, and respect when caring for the Mexican American woman, the health care provider will be able to provide assessment, screening and health promotion activities to promote better outcomes (Mahon, McFarlane, \& Golden, 1991; Zoucha \& Zamarripa, 2008).

Familialism is one of the most important culture-specific values of Hispanics. It is an allencompassing value among Mexicans and takes precedence over all aspects of life (Zoucha \& Zamarripa, 2008). Familialism is the value that involves individuals' strong identification with and attachment to their nuclear and extended families, and strong feelings of loyalty, reciprocity, and solidarity among family members (Marin \& Marin, 1991). In an ethnographic study, Niska (1999) 
found that nurturing in the Mexican American family was characterized by being kin-based and intimate in nurturing of the family. Material support focused mostly on household needs and emotional support was sharing stories, conversation, problem solving, and prayer. Socialization within the family was kin-based, hierarchical, and ritualistic. This strong family support produces a love for children and reinforces the importance of the mother's role in nurturing and protecting the child before and after birth (Negy, 1993).

Basic internal family systems (cohesion, expressiveness, conflict, organization, and control) of famililialism tend to remain intact even in third generation acculturated families. The external family systems increased as acculturation changed. The more Americanized in ethnicity they were the more primary and secondary kin they had on hand and with whom they frequently visited and remained close (Keefe, 1980; Rueschenberg \& Buriel, 1989; Negy, 1993). This suggests that acculturation modifies only certain aspects of family relationships among Hispanics (Marin \& Gamba, 2002).

Motherhood is highly valued within the Mexican culture and pregnancy is considered a vulnerable time. Most women return to traditional health practices to protect the developing fetus. Female relatives give instructions for support: rest, a healthy diet, and avoidance of stress and worry. They pamper and cater to the woman to ensure optimal pregnancy health. The woman's work is adjusted and supportive female family members often take over housework as the pregnancy progresses (Negy, 1993; Langará \& Gonzalez-Rameriz, 2003).

Much of the literature has emphasized a devotion of the Mexican American woman to motherhood and childbearing. Amaro (1988) conducted interviews with 137 Mexican-American women visiting a community health center. He wanted to know the reproductive attitudes and behaviors of these women, and investigated the relationship of these attitudes and behaviors to socioeconomic status, acculturation, and religiosity. Overall, his results contradict the stereotype that Mexican-American women are passive in fertility decisions, desirous of large families, and dominated by Catholic doctrine. 
Even though the Mexican American women are portrayed as super-mothers, dictated into submissiveness and continuous reproduction by cultural and religious traditions, Amaro's study showed a great heterogeneity. This was true even in relatively low-income and unacculturated Mexican American women. It was clear that motherhood was important to the majority of them but most wanted a moderate family, and many used contraception. He suggested there is a need for research to understand how Mexican American women experience interactions with the health care system, with their partners, and with social institutions like religion. The use of fertility rates and epidemiological data alone will not provide the information needed to become familiar with the beliefs, experiences, and problems of these women and to design the services to respond to their needs (Chun \& Akutsu, 2002).

Guendelman, Malin, Herr-Harthorn, and Vargas (2001) explored the attitudes and values that shape Mexican women's perceptions of motherhood. The researchers used focus groups with 60 pregnant low-income rural women in Mexico, immigrant women from Mexico living in the United States, and United States born women of Mexican descent living in urban California. The orientation to motherhood changed with access to social and economic resources as well as changes in cultural values. Participants from rural Mexico generally chose the conventional discourse of motherhood and domesticity. The immigrants in California chose either the conventional discourse or one of working mother, depending on their financial resources. The Mexican American women's partners more often encouraged them to return to work or school after birth. These women showed an emphasis on individualization and self-development. Motherhood while still cherished, whether planned or unplanned, was not life defining as with the other two groups.

The daily-lived experience of a group of low-income working Mexican women in their maternal and spousal roles was interpreted within the context of the cultural context of the Mexican family and the interplay of machismo and hembrismo. Machismo is understood from the stereotypical view of the unquestionable and absolute supremacy of the male and the inferiority of 
the female. Hembrismo characterized by a gender division of roles gives power and authority to women within their domestic sphere. The male and female achieve their social status and emotional support from the family, not from their jobs as other cultures often do (Meleis, Douglas, Eribes, Shih, \& Messias, 1996).

The female is at the center of the Mexican family and receives honor and prestige. A higher status is provided for women with children than non-mothers, but it does not exempt the female from the stress of the role. This combination of domestic and work roles may have an adverse effect on women's health. Their resources identified in the study were their work, children, extended family, and their inner strength and resilience that enabled them to have a certain degree of control over their lives (Meleis et al., 1996).

Mexican American childbearing women appear to represent a healthy model comprised of many protective behaviors for prevention of preterm and lower birth weight. (Becerra, Hogue, Atrash, \& Perez, 1991; Guendelman \& Abrams, 1995; Jones \& Bond, 1999; Balcazar, Krull, \& Peterson, 2001; Laganá, 2003). Acculturation and perinatal outcomes studied over the last two decades have confirmed an epidemiological paradox between acculturation and perinatal outcomes. The epidemiological paradox refers to the idea that protective behaviors of the woman provide lower infant mortality. The researchers proposed that the paradox observed in Mexican American Women is due to cultural orientation.

Jones and Bond (1999) studied 382 Hispanic origin women, reporting acculturation status, and their birth outcomes. The findings support the hypothesis that traditional Mexican cultural practices serve to protect the childbearing woman and suggest that family and family social support contribute to this positive perinatal outcome.

Balcazar, et al (2001) suggested that higher levels of family cohesiveness foster the most constructive health and psychosocial outcomes. Higher levels of family cohesiveness resulted in less coping and a lower quality of prenatal care for the less acculturated women in some circumstances. 
Sagrestano, Feldman, Rini, Woo, \& Dunkel-Schetter (1999) looked at ethnicity and social support during pregnancy of groups of African American, Latina, and non-Hispanic White women. Several types of support (perceived support, received support, support satisfaction, and network support) and sources of support (baby's father, family, and friends) were assessed. After controlling for demographic variables, ethnicity did not predict support from the baby's father, but marriage was the most important predictor of support from the baby's father. Support from friends and family was complex and associated with ethnicity, socioeconomic status, age, parity, and marital status. The authors noted that more research is needed examining the contribution of psychosocial factors to ethnic variability in birth outcomes. Contextually relevant demographic variables and social support measures differentiate between types and sources of support. The authors note that a limitation of the study was the inability to test the impact of culture on ethnic differences in social support. This needs to be included in the future studies. Although translated, the validation of the tool they used was with college students, who may differ from pregnant Latinas.

In a study of African American and Latino adolescents, Florsheim, Sumida, et al (2003) wanted to identify predictors of parental functioning among these young couples. They examined ethnic differences in the couples. How the couples adjust to parenthood was found to depend on culturally specific norms, expectations regarding the couple's relationship, and their parenting behavior.

In another study with adolescents, Becerra and de Anda (1984) studied 122 adolescents between the ages of 13 and 20, who were pregnant at the time or delivered within the previous twelve months. The majority of the adolescents were Mexican American (82) and the others were White non-Hispanic (40). Of the Mexican Americans, 39 were Spanish speakers. The researchers used a closed-ended questionnaire to gather data related to the adolescents' social networks, perceptions of their families' attitudes, attitudes toward themselves, and knowledge about reproduction and contraception. The findings suggested that acculturation level of the 
adolescent can influence the role that family and peers play in her life. It can influence the impact these individuals may have on her attitude about herself and her knowledge about the use of contraception and her own fertility. The Mexican American adolescent mother who is in the process of becoming acculturated is caught between two worldviews. The English-speaking Mexican American adolescents were more at risk for unwanted pregnancies and illegitimate births than other groups of adolescents. This group did have a strong emotional and material support system and good communication with their mothers and a continued relationship with the father of the baby. The Spanish-speaking Mexican American adolescent mothers were not as likely to have available the support of family and peers and did not demonstrate the knowledge regarding reproduction and contraception. Often they were isolated geographically from their families of origin. Cultural differences and the differences in knowledge as affected by where an individual is in her acculturation process is a key implication in strategies of prevention and intervention.

Engle, Scrimshaw, Zambrana, and Dunkel-Schetter (1990) studied the Mexican woman (96\% born in Mexico) who was giving birth to their first child. They examined psychosocial factors related to prenatal and postnatal anxiety, and hypothesized that the less acculturated the woman, the higher her prenatal anxiety about labor and delivery. The prenatal and postnatal interviews focused on topics such as anxiety levels, acculturation, and knowledge regarding childbirth, social support, and the desire for control during labor and delivery. Acculturation was not directly associated with prenatal or postnatal anxiety. Decreased prenatal anxiety was attributed to assertiveness, a desire for control during labor and delivery, and social support from family and friends.

Appreciation of the beliefs, values, and practices related to the childbirth experience prompted Callister and Vega (1998) to conduct an ethnographic study on the cultural beliefs of giving birth for Guatemalan women. Three themes emerged: a sense of sacredness of giving birth; the need to rely on religion during the pregnancy, childbirth and childrearing; and the 
paradox of childbirth as bittersweet (an extremely painful experience but a joyous experience). The researchers conducted the study in Guatemala to gain a better understanding of the Central American refugees and immigrants in the United States. Callister has done extensive research in North and Central America, Scandinavia, the Middle East, and China. From her research, she has noted the strength of the women. It is important to build on the strengths of the culturally diverse woman rather than their deficits (Callister, 2001). She noted that "nurses who are willing to learn about their client's culture by giving women the opportunity to share their perceptions of the meaning of childbirth may be more successful in promoting positive physiological and psychosocial outcomes" (Callister, 1995, p. 293).

\section{Summary}

This chapter began with a historical overview of mothering. Deutch in 1945 first described motherhood as the relationship to her child while Benedek in 1956 suggested that mothering refers to the practical aspects of caring for the child. Mothering is the quality of the woman's personality that provides the energy to accomplish the care of the child. These early theorist provided the basis for later frameworks by Rubin and Mercer in developing their I frameworks for attainment of the maternal role attainment and becoming a mother. These frameworks provided the inspiration for the framework for this study. Although extensive research has been done using these frameworks, the need for a greater understanding of mothers from other cultures is needed. The Psychosocial aspects of pregnancy and the components of the beginning aspects of mothering during pregnancy, attachment, commitment, and preparation provided a basis for further study in this area. The studies discussed that mothering is a process but much of the research has focused on childbirth and the mother-child relationship and has not focused on the pregnancy period and the beginning process of mothering. 
A demographic overview of Hispanics in the United States showed the affects of migration, immigration, and acculturation on how these families have their needs met. An extensive review of the importance of culture on mothering was included in this chapter. Studies on the Hispanic population have included both quantitative and qualitative studies concentrating on psychosocial factors, cultural meaning of social and partner support, maternal adaptation, the importance of family, and the affect of acculturation on this process. Further investigation is needed to delineate different cultures within the Hispanic population. As noted in the literature, each group within this population is unique and warrants separate exploration. The Mexican origin woman's prenatal fetal attachment, their commitment to the pregnancy, and the need for preparation provides an opportunity for further study. This study began to fill this gap in knowledge. 
CHAPTER 3

\section{METHODS AND PROCEDURES}

This chapter will describe the design for conducting the study with the Mexican origin woman during her pregnancy. Discussion will focus on the design and method, sampling procedure, setting for recruitment and interviews, data collection, procedure, ethical considerations, data analyses, and delimitations.

\section{Research Design}

A qualitative, descriptive inquiry was conducted to explore the anticipatory stage of becoming a mother in the Mexican origin woman. Descriptions of the participant's experiences are an appropriate way to study human action. Qualitative research methods describe the social world from the perspective of the participants (Lincoln \& Guba, 1985). This design allowed for discovery and documentation of participants' personal perspectives and views, and a study of real-world situations as they emerged, unfolded, and developed naturally (Lincoln \& Guba, 1985). The qualitative descriptive method is the method of choice when what is desired is straight description of the phenomena (Sandelowski, 2000). The description of the attachment, commitment, and preparation phases during the anticipatory stage of becoming a mother, requires the perceptions, inclinations, sensitivities, and sensibilities of the Mexican origin woman. This study begins to interpret this experience. Semi-structured interviews provided direct, indepth responses about the woman's experiences, perceptions, feelings, and knowledge of becoming a mother. The data consisted of verbatim quotations (Patton, 2002). An emic or insider's point of view (Spradley, 1979) allowed the woman to provide her experience of pregnancy and the meaning she attaches to those experiences as she sees them. 


\section{Sample}

Purposive and criterion sampling was used to identify the study participants. Purposive sampling selects information-rich cases (Patton, 2002). These cases are those that can provide a great deal of information about issues of central importance for the inquiry. Criterion sampling occurs when a set of criteria is selected and provides rich data relevant to the research problem (Patton, 2002; Liamputtong \& Ezzy, 2005).

The sampling criteria required that the participant to be:

1. a self-identified Mexican origin woman,

2. twenty-five to thirty weeks gestation at the time of the interview, to allow for entrance into prenatal care and to avoid the threat of miscarriage to not be a concern,

3. between the ages of 18 and 22 ,

4. receiving prenatal care at one of two prenatal clinics,

5. either monolingual English or bilingual, English/Spanish, and

6. a primigravida.

Marital status or complications of pregnancy were not a reason for exclusion.

\section{Definitions}

For the purposes of this study, the following definitions were used when identifying the participants:

1. Mexican origin refers to those individuals residing in the United States, born in or tracing their family to Mexico.

2. Gestational age was calculated using a gestational wheel and risk management timetable with their due date as the point of reference.

3. Age was calculated by their date of birth.

4. Prenatal care is care received by a health care provider during pregnancy and before delivery.

5. Primigravida is a pregnant woman who has not delivered an infant previously. 
Saturation of the data determined the sample size. Since purposive sampling aims to create rich, in-depth information, termination of sampling occurs when no new information is being received, redundancy being the primary criterion (Liamputtong \& Ezzy, 2005). When analysis produces no new themes, categories, or variations in the descriptions, the final sample size was determined. To attempt to saturate the categories and find all variations of this stage of mothering, recruitment sought a variety of women meeting the criteria but of different ages and weeks gestation within the sample criteria.

\section{Setting}

Recruitment of participants for this study occurred from two prenatal clinics and one Catholic Church. The initial recruitment was from an academic clinic which is affiliated with a health care system providing health care for disadvantaged families in the southwest area of a large metropolitan city. The clinic provides prenatal care to a diverse population of women from the community. Recruitment continued at a federally funded community health center that has served the community for over 30 years. The patients are drawn from all areas in the metropolitan area but the core is an area near downtown that is predominately Hispanic. The staff is bilingual English/Spanish and is often of the same culture as the patients. A third site was a large Catholic Church predominately serving Hispanic parishioners.

\section{Data Collection Method}

\section{Demographic Questions}

The Mexican origin woman was the primary source of the data. The interviewer and researcher used a short form to record the demographic data (Appendix B) The demographic data consisted of age, due date, marital status, who living with, if working or in school, birth place, how long lived in United States, if Mexican origin, and language spoken at home.

\section{Interview Questions}

The semi-structured interviews provided the primary data in this study. Design of the questions provided the direction to obtain information to answer the study questions. Descriptive 
questions allowed the woman to talk about being pregnant and becoming a mother. Spradley (1979) describes these questions as especially useful to start the conversation and to keep them talking. These questions encourage persons to talk about a particular cultural scene and to "ramble on and on" (p. 87). Women generally enjoy discussing their pregnancy, so beginning questions establish trust and comfort and allow the interviewer to address questions that are more difficult.

Semi-structured starter questions with the related concept are presented in Table 1. The definitions of the concepts and the model provided in Chapter 1 and the literature in Chapter 2 provided the basis for the content of the questions. The model is based on the work of Rubin, Mercer, and the later work by other researchers such as Walker, Crain, Thompson (1986a), Koniak-Griffin (1993), Lederman (1996), and McBride and Shore (2001). These provided the background for the questions. The literature on the development of the maternal fetal attachment tools (Cranley, 1981; Müller, 1996) provided further information on attachment. Additional literature on the importance of culture on mothering assisted in formulating the questions related to culture, family, friends, and their partners. Spradley (1979) and Marin and Marin (1991) provided resources for culturally appropriate questions. Questions were refined and added after each interview was transcribed and analyzed.

Table 1. Sample Semi-structured starter questions

\begin{tabular}{|l|l|}
\hline \multicolumn{1}{|c|}{ Concept } & \multicolumn{1}{|c|}{ Simi-structured Sample Starter Questions } \\
\hline Overall & Who lives with you in your house? \\
\hline & Are you working or in school? \\
\hline & $\begin{array}{l}\text { Could you describe the main things that happened from the } \\
\text { beginning of the pregnancy until now? }\end{array}$ \\
\hline & Tell me about your life now. \\
\hline & What do you think is the best thing about being a parent? \\
\hline
\end{tabular}


Table 1. Continued

\begin{tabular}{|c|c|}
\hline & What do you think is the worst thing about being a parent? \\
\hline \multirow[t]{3}{*}{ Attachment } & $\begin{array}{l}\text { What do you think about most often when you think about the } \\
\text { baby? }\end{array}$ \\
\hline & $\begin{array}{l}\text { What do you think about when you think of the baby as a boy } \\
\text { or girl? }\end{array}$ \\
\hline & What do you think most often about the baby? \\
\hline \multirow[t]{6}{*}{ Commitment } & What has the pregnancy been like? \\
\hline & Are there any concerns about your pregnancy? \\
\hline & Describe a typical day after the baby comes. \\
\hline & What do you think your life will be like after the baby comes? \\
\hline & What type of mother do you think you will be? \\
\hline & Describe that mother. \\
\hline \multirow[t]{3}{*}{ Preparation } & Describe a day that you imagine you are caring for the baby. \\
\hline & $\begin{array}{l}\text { Do you intend to attend or are you presently attending } \\
\text { expectant parenting or labor preparation classes? }\end{array}$ \\
\hline & $\begin{array}{l}\text { Whom do you ask questions about your pregnancy and your } \\
\text { baby? }\end{array}$ \\
\hline \multirow[t]{3}{*}{$\begin{array}{l}\text { Culture, family, friends, } \\
\text { partner }\end{array}$} & $\begin{array}{l}\text { I'm interested in how you and other women in your family talk } \\
\text { about your pregnancy and the baby. }\end{array}$ \\
\hline & $\begin{array}{l}\text { I'm interested in how other women in your family help you } \\
\text { during your pregnancy and how they will help you after the } \\
\text { baby comes. }\end{array}$ \\
\hline & $\begin{array}{l}\text { When talking to your mother during your pregnancy, what } \\
\text { kinds of things do you discuss? }\end{array}$ \\
\hline
\end{tabular}




\section{Procedure}

Recruitment was proposed to occur at two clinics. Recruitment at the initial site (academic clinic) only provided two patients that met the criteria over a period of several months. The number of Mexican origin patients who were the appropriate age and gravidity was limited. Neither of the women recruited from this site participated in the study because they did not answer the phone when called for their interview. Recruitment at other sites was initiated after several months when no women from this site participated. Recruitment at the church occurred Sunday during the announcements at the end of the six services with the priest calling for participants. No participants were recruited at this site.

At the community health center, recruitment occurred during the women's prenatal visit. Clinic personnel reviewed the charts and asked the women who met minimum criteria if they were willing to meet with the researcher. Twenty-one women were recruited over a period of six months and 20 were enrolled in the study. One woman did not wish to participate at the time of recruitment, one at the time of the interview, and 9 either did not answer their telephone when called or did not return to the clinic for care. Ten women participated in the interview process.

The procedure for the data collection is outlined in the flow chart (Appendix C). The clinic staff reviewed the chart and approached the possible participants who met the initial minimum requirements of age and gestational age, and of Mexican origin. The researcher interviewed the prospective participant for assurance that she met inclusion criteria and evaluated her English language skills. Once the woman stated that she met the criteria, the researcher explained the study in full and read the consent with her. The woman then had the opportunity to ask questions for greater understanding. The consent process followed the requirements of The University of Texas at Arlington, Office of Research Compliance, and the facility where recruitment occurred. An explanation of the study purpose was given. The basic aspects of the informed consent included statements regarding the research, purpose, duration of participation, and procedures. After she agreed to participate, she signed and received a copy of the consent. Prior to 
beginning the interview, while recording, the participant again confirmed that she agreed to be a part of the study and be recorded.

The researcher and participant arranged a convenient time to conduct the interview, either by phone or face to face during a clinic visit. The participant's telephone number was obtained for a later contact. Of those who did not participate or were lost to the study, there was no further contact after the researcher made two calls without a response. They did not wish to participate at the interview time, or they did not return to the clinic for prenatal visits.

For the ten women who participated, the researcher started the interview by asking the demographic questions to set the stage and to assist in creating a comfortable environment. The interview took approximately 20-40 minutes. After the initial interviews (one by phone and one face to face), the participants and researcher met to allow the woman to review the data and to offer any clarification about her interview. In each of the other eight face to face interviews clarification occurred at the conclusion of the interview. They were given an opportunity to share additional information if they wanted at the end of the interview or during a later meeting. None of these women wanted to meet later, although four of the women agreed to be available for clarification if the need arose. Collection of information was not collected later in their pregnancy because of a possible alteration in how they might answer the questions at that time. At the final meeting, she received a gift certificate of $\$ 25.00$ from a retail store for her time.

Measures to ensure credibility during data collection included member checks, reviewing transcribed interviews with an experienced researcher of Hispanic women, and an audit trail ( Appendix D). Since the researcher was not of Mexican origin, the interviewer and clinic staff provided the cultural link for these women and created a sense of trust. Being at the clinic during prenatal visits provided an opportunity for the women to see and meet the researcher and be more at ease during the interview process. To maintain rigour during data collection the researcher used purposeful sampling, asking questions that were literature based, changing the way data was collected when the collection method was not working, and continuing collection of 
data until saturation occurred (Cutcliffe \& McKenna 1999; Morse \& Richards, 2002; Tobin \& Begley, 2004).

After the first two interviews, the transcriptions were reviewed. One interview was conducted by telephone using the Mexican origin interviewer and one was face to face with the researcher without a Mexican origin interviewer present. During the telephone interview, the researcher realized there were lines of questioning the Mexican origin interviewer felt were not culturally appropriate to pursue. The researcher reviewed the questions with the interviewer for subsequent training for use during additional telephone interviews. However, the Mexican origin interviewer was not able to conduct interviews at the clinic due to a conflict with her work schedule and the remaining interviews were conducted face to face by the researcher.

Data management began at the beginning of data collection. All information related to a particular participant was kept together until the analysis was complete. Field notes began during the initial communication with the participant and continued until after the final meeting with the woman. An audit trail (Appendix D) made it possible to track the data collection process.

Ethical Considerations

\section{Review Process}

Methodist Health System, is responsible for the requirements for the Institutional Review Board (IRB) requirements and reviewed the research protocol. The following forms are included in Appendix E:

\section{Informed consent in English}

2. Approval letter from Methodist Health System.

The University of Texas at Arlington, Office of Research Compliance is responsible for the Institutional Review Board (IRB) requirements and reviewed the research protocol. The agency where recruitment and interviews occurred gave permission to conduct the research study at their site. The following forms are included in Appendix E: 
1. Informed consent in English,

2. The initial approval letter from The University of Texas at Arlington,

3. The approval letter for change from The University of Texas at Arlington, and

4. An approval letters from the agency and the church.

\section{Potential Risks}

Although the potential risk was minimal, there was the possibility for the participant to experience psychological discomfort while discussing her thoughts and feelings about becoming a mother. The participants received a thorough explanation of the nature and purpose of the study so they understood the reason for the questions and understood that they were free not to answer any question that they did not wish to discuss. During the telephone interview, the interviewer was not able to observe visual clues that the individual was uncomfortable; she listened for verbal cues and verbally validated with the participant her impressions, asking if the participant wished to continue. When doing the interview face to face, the researcher observed for signs of discomfort during questioning and either asked for clarification, discontinued the line of questioning, or returned to the subject later in the interview using another form of the question. Benefits

The main potential benefit from this study is the acquisition of knowledge regarding how women of Mexican origin describe becoming a mother during the pregnancy stage. This study did not have a particular benefit to the participants. Participants were compensated financially with $\$ 25.00$ in the form of a Target gift card after the final meeting.

\section{Possible Adverse Events}

During the interview process, the participant may experience possible psychological distress. As noted earlier, the researcher allowed the woman to pause during the interview to collect herself and then to proceed or discontinue the interview. She was encouraged to share her concerns with her health care provider. During the interview processes, the participants made no reference to violence in the home, so there was no need for a referral. 
Informed consent

To establish eligibility for the study, the researcher explained the study, and asked if she was Mexican origin. Once the woman stated that she met the criteria, the researcher explained the study in full and read the consent with her. She had the opportunity to ask questions for greater understanding. The consent process followed the requirements of The University of Texas at Arlington, Office of Research Compliance, and the facility where recruitment occurred. An explanation of the study purpose was given. The basic aspects of the informed consent included statements regarding the research, purpose, duration of participation, and procedures. After she agreed to participate, she signed and received a copy of the consent. Prior to beginning the interview, while recording, the participant again confirmed she agreed to be a part of the study and recorded.

\section{Data Analyses}

The demographic data were kept in an excel file where simple descriptive summaries were calculated. Sums and percentages were run for the purposes of review of the characteristics of the women interviewed. The data were arranged in a table by the questions asked. Each of the ten participants was given a subject number.

Data analysis began and continued after each interview. Qualitative content analysis was best suited to elicit meaning from the data and permit themes to emerge from this descriptive study. From the data, codes were consistently applied and generated. The characteristics of qualitative research are the simultaneous collection and analysis of data where both shape each other. Accommodation of new data and insights occurred as modification continued. Latent content analysis, the process of identifying, coding, and categorizing the primary patterns in the data, captured the meaning of the passages within the content of the data (Mayan, 2001).

The researcher listened to the digital recording in its entirety to capture the fundamental or main significance of the conversation as a whole. Key phrases were noted and field notes reviewed. The researcher transcribed each interview. An independent reviewer reviewed each 
transcribed interview for accuracy of transcription by listening to the digital recording while reviewing the transcript. The format used for the transcript presented the interview verbatim indicating when the interviewer or woman was talking. Each was coded with the participant's subject number and date of interview.

Coding began during the initial read. The researcher read and re-read, highlighted sections, and made comments in the margins. Analysis was in the context of the Anticipating Mother model and included coding by line and section for key ideas. The data were arranged in a table format with theme, code number of participant, line number in transcription, and the quote.

The integration of the categories occurred by looking at the big picture and considered the data and the themes. By looking at how the categories are related, what patterns kept occurring, and what conclusions were drawn allowed identification of the themes. These themes told the big picture about the data (Mayan, 2001).

A second experienced qualitative researcher coded two of the interviews. Once completed, the experienced coder and the researcher compared and worked together to obtain consensus on themes that best represented the words of the women as their own.

Transferability refers to whether the findings are relevant to similar contexts (Pitney, 2004). Using the demographic data, interview data, field notes, and observations, a thick detailed presentation of the woman's description of becoming a mother allows others to determine for themselves whether the findings allow use by others. Finding common themes among the women might suggest to others that the findings are applicable to their group.

The woman verified the accuracy of her information discussed during the interviews either during a subsequent prenatal visit or during the time of the interview. Review of the data assured that the data captured the true essence of her descriptions.

Assumptions of the analysis

Qualitative descriptive design (Sandelowski, 2000):

1. entails interpretation of results in greater consensus among researchers, 
2. presents the facts in everyday language,

3. collects as much data as necessary to capture the elements of the event,

4. offers a summary of an event in everyday terms of the event,

5. language is a vehicle of communication, not an interpretive structure.

Rigor and Credibility of Analysis

Conduction of verification strategies ensured rigor of the study. Verification is checking, confirming, making sure, and being certain. The data were collected and analyzed concurrently to form a mutual interaction between what is known and what one needs to know. Moving back and forth between data collection and analysis allowed systematic checks of the data, maintained focus, the fit of the incomplete thoughts, and the conceptual work of analysis and interpretation monitored and confirmed (Mayan, 2001). As noted in the analysis process, the following strategies used during the research contributed to rigor and credibility (Mayan, 2001):

1. Participant checks were conducted during and at the end of the interviews. Further verification by the participants did not occur since the participant was moving through her pregnancy and this might alter the data. The verification helped to ensure credibility and assure their message was received. The researcher conducted the participant checks.

2. Journal writing served to keep the process honest. This journal provided a record of personal biases and assumptions about the research and how the interview process was progressing. The journal included information on how the researcher felt the interview went and the comfort level of the woman during the interview.

3. The researcher created an audit trail to show a decision trail so that others are able clearly to follow the analysis process (Appendix F).

4. The dissertation chair (a knowledgeable researcher of Mexican culture) reviewed decisions, conjectures, and made suggestions for possible future steps.

5. An independent researcher coded and validated two interviews and met with the researcher to arrive at mutual interpretations and meanings. 


\section{Anticipated Outcome}

Presentation of the data was in the form of a descriptive summary of the anticipatory stage of becoming a mother for this group of Mexican origin women. This qualitative descriptive study describes the phenomena of anticipating mothering for these women.

\section{Delimitations}

Delimitations for this study described the population that would be studied. Following are the delimitations:

1. The study excluded any woman who did not receive care at on specific clinic in the Southwest are of Dallas, Texas (Los Barrios Unidos Community Clinic, Inc.).

2. The study excluded any woman who did not self-identify as of Mexican origin.

3. The study excluded any woman who was not twenty-two to thirty weeks pregnant at the time of the interview and a primigravida.

4. The study excluded any woman who was not between the age of 18 to 23 years.

5. The study excluded any woman who was not either monolingual English or bilingual, English/Spanish.

\section{Summary}

This descriptive qualitative inquiry explored the anticipatory stage of becoming a mother for a group of Mexican origin women. Interviews using semi-structured questions by telephone or face to face with the women twenty-two to thirty weeks pregnant, 18 to 23 years of age provided the data presented in Chapter 4. Discussion of recruitment strategies, informed consent, the procedure for data collection and analysis provided in this chapter gave credibility to the study method. The method and procedures presented in this chapter provided the groundwork for the presentation of the emic point of view of the women discussed in the following chapter. 
CHAPTER 4

\section{FINDINGS}

Organization of the thematic findings occurred using the constructs of the model Anticipating Mothering in the Mexican Origin Woman shown in Figure 1: (1) attachment, (2) commitment, and (3) preparation. The chapter begins with the participants' characteristics in Table 2. The themes within the constructs that emerged are presented with exemplar quotes. Three additional themes emerged; determination to succeed, strong woman, and good mother and represent the researcher's interpretations of the women's comments and the information they shared. Findings with pertinent research provided along with limitations and the conclusions gave background for the implications for nursing and recommendations for further research.

\section{Sample Characteristics}

Twenty women initially met the criteria and agreed to participate in the study. There were varied reasons that some did not complete the interview. Three of the women did not answer the phone when called after initially setting the time of the interview. One requested her interview in Spanish even though she communicated in English with the researcher to sign the consent and give permission. One developed problems with her pregnancy, transferred to a high-risk clinic, and no longer met the criteria. Two declined to do the interview when their gestational age was between 23-30 weeks and two were lost to the clinic after missing several prenatal appointments. Table 2 displays the characteristics of the 10 women interviewed. All women identified themselves as of Mexican origin either being born in the United States or living here for 6 to 20 years. The majority of the women were 18 years of age. The one woman who was 23 was 22 at the time of the initial screening interview and signing of her consent, having a birthday two weeks prior to her interview. 
Table 2. Characteristics of Participants $(\mathrm{N}=10)$

\begin{tabular}{|c|c|c|}
\hline Characteristic & Frequency & Percent \\
\hline \multicolumn{3}{|l|}{ Age } \\
\hline 18 & 7 & $70 \%$ \\
\hline 19 & 1 & $10 \%$ \\
\hline 20 & 1 & $10 \%$ \\
\hline 23 & 1 & $10 \%$ \\
\hline \multicolumn{3}{|c|}{ Gestational Age (Weeks) } \\
\hline 23 & 2 & $20 \%$ \\
\hline 25 & 2 & $20 \%$ \\
\hline 26 & 1 & $10 \%$ \\
\hline 28 & 1 & $10 \%$ \\
\hline 29 & 2 & $20 \%$ \\
\hline 30 & 2 & $20 \%$ \\
\hline \multicolumn{3}{|c|}{ Years in the United States } \\
\hline 6 & 2 & $20 \%$ \\
\hline 9 & 1 & $10 \%$ \\
\hline 11 & 1 & $10 \%$ \\
\hline
\end{tabular}


Table 2. - Continued

\begin{tabular}{|c|c|c|}
\hline 12 & 1 & $10 \%$ \\
\hline 13 & 1 & $10 \%$ \\
\hline 18 \& 20 (born in U. S.) & 4 & $40 \%$ \\
\hline \multicolumn{3}{|l|}{ Language spoken at home } \\
\hline English & 1 & $10 \%$ \\
\hline Spanish & 5 & $50 \%$ \\
\hline Both & 4 & $40 \%$ \\
\hline \multicolumn{3}{|l|}{ Working } \\
\hline Yes & 4 & $40 \%$ \\
\hline No & 6 & $60 \%$ \\
\hline \multicolumn{3}{|l|}{ School } \\
\hline Yes & 3 & $20 \%$ \\
\hline No & 7 & $70 \%$ \\
\hline \multicolumn{3}{|l|}{ Level of Education } \\
\hline Graduated High School & 7 & $70 \%$ \\
\hline Attending High School & 3 & $30 \%$ \\
\hline \multicolumn{3}{|l|}{ Marital Status } \\
\hline Married & 3 & $30 \%$ \\
\hline Common Law & 1 & $10 \%$ \\
\hline Single & 6 & $60 \%$ \\
\hline \multicolumn{3}{|l|}{ Living With } \\
\hline Aunt & 2 & $20 \%$ \\
\hline
\end{tabular}


Table 2. - Continued

\begin{tabular}{|c|c|c|}
\hline Parents & 2 & $20 \%$ \\
\hline Mom & 2 & $20 \%$ \\
\hline Mom and husband & 1 & $10 \%$ \\
\hline Parents and husband & 1 & $20 \%$ \\
\hline Husband & 2 & $80 \%$ \\
\hline Partner involved & 8 & $20 \%$ \\
\hline Yes & 2 & \\
\hline No & & \\
\hline
\end{tabular}

Themes within Constructs

The themes are presented using the constructs of the model "Anticipating mothering (pregnancy) in the Mexican origin woman". The emergent themes are discussed as separate from the model constructs and how they might become an integral construct in a future model. The quotes with the subject's number provide the data. The characteristics of each subject are presented in Appendix G.

\section{Attachment}

Attachment as described in Chapter 1 begins with the idea of being pregnant and slowly moves to attachment to the fetus (Rubin, 1984). The relationship leads to the future acceptance, nurturance, and protection of the child (Leifer, 1980; Muller, 1990; Mercer, 2006). The young women described feelings about being pregnant and becoming mothers. The themes that emerged related to attachment were uncertainty, worries/joys; fantasies/dreams, and unplanned/acceptance 
Uncertainty/Worries/Joy

The young women noted that they were scared or worried when they found out they were pregnant. "Well, I was nervous when I first got pregnant. I try to be positive, but yeah, there was negative stuff cause l'm a student. I'm still in high school" (Subject 3). Her boyfriend was involved now, but it had been a question about their relationship when she originally told him about the pregnancy. She lives with her aunt. Her parents are in Mexico. One person expressed the idea that the pregnancy was "scary and at the same time we shared happiness." Another woman was affected by the reaction of her family and boyfriend. "I was happy at first; it didn't work out the way I wanted it to. The way my family reacted and the way my boyfriend reacted" (Subject 6). Her boyfriend left for Iraq shortly after learning of the pregnancy and there was not time to get married like she and her parents wanted. One of the women showed how pregnancy brings concern and excitement at the same time. "It was exciting at first, a little bit nervous, because it was my first time, so it was kind of exciting and nervous at the same time. I felt good, I felt good" (Subject 14).

Others were concerned that everything would be all right. "The only thing that I want is for it to come out right" (Subject 11). One discussed a decision about a test for

some syndrome or something, that's the only thing that worried me, but I'm a really religious person and if God sees my baby the way he wants it, I'm gonna accept it so (Subject 10).

Most women expressed joy when talking about their baby. Even when they had concerns about being pregnant, they expressed joy and excitement when talking about their pregnancy, the baby, and what their life would be like: "I'm excited about it" (Subject 4) and "I'm just so happy" (Subject 11). One talked about the joy of talking to her baby, "I like to talk to him a lot, you know a person's talking about things" (Subject 17). They also expressed joy when they described how they felt when they started feeling fetal movement and having changes in their body.

It feels great, it's wonderful. There's times that you're by yourself and you feel your stomach moving, it's just great, you feel great (Subject 9). 
My stomach started feeling more different. There was changes in my body. It was actually a beautiful pregnancy (Subject 9 ).

It feels great, like you're feeling your baby and it's moving and you know he's fine (Subject 17).

Some expressed their joy by stating how being a mother was about to change their lives. They thought it would be a good change even if it were hard.

I think it's just something; it's going to change the whole atmosphere of the house. It's going to be one more family and I don't know, it's just something that I can't explain. It's just a mother, I don't know that [mother] feeling.

You're going to have your baby there and expecting the best of you (Subject 20).

I think it will make a big change because to love a stranger from one day to another and decide what's good for them without him or her being able to tell me anything about it (Subject 4).

Awesome! To actually have it, to have my baby, not just my nephew, you know. It's going to be my baby and I'm gonna take care of it and not my mom, not my sisters; me and my husband (Subject 10).

One of the women was not sure that it would be a joyful occasion. She was afraid "that everybody's going to be with the baby now and not with me" (Subject 17).

Fantasy/Dreams

Fantasizing about the baby is an important aspect of the attachment phase of pregnancy. According to Rubin (1984), the mother fantasizes about her baby as an older child. All of the women expressed they wanted to "see" what the baby looked like but a few were unable to verbalize what they were going to do with him. "I want to see what he looks like, and stuff like that but not what I'm gonna do with him. I can't think about it" (Subject 6). Many stated that they had dreams about the baby and saw the baby a certain way. Others were unable to see how the baby looked only what he/she was wearing. "When I dream, I see myself with my baby inside, it's a boy" (Subject 9). One was able to describe the baby and was excited and anxious about seeing what the baby looked like.

Spending time with her, watching her growing up, and stuff like that. Physically she's kind of light skin, dark hair, with a big lips, big eyebrows, eyes, big eyes and brown. I just think about her physically, I just want to see her out, what she 
looks like. I'm more anxious about that (Subject 14).

Well black hair, I see it like my husband's face. Sort of..that's what I feel, but don't Know (Subject 20).

Several had dreams that the baby was a certain gender. This woman's dream showed her baby as a boy because of how the baby was dressed. One described her baby as "a little angel. I think it's a girl" (Subject 20).

I had a few dreams, I didn't know it was a boy, I had like two dreams that it was a boy, like how he was dressed, like buying the clothes and stuff. So I knew it was a boy. But I wonder how he looks like. I couldn't see [how he looked]. I was right [it was a boy from the sonogram] (Subject 17).

I'm thinking moms know, kind of in a way, what you're going to have but l'm not sure. Me, I'm thinking, l'm leaning more to that it's going to be a boy (Subject 9).

Others had dreams where they saw their baby much later in life playing sports, watching them grow, and calling them mother.

I think it's going to be beautiful...you'll hold his hand, he's already three years old, and he starts asking you questions 'mom what is that, mom what is this?' And the first day of school is gonna be great too (Subject 9).

I want to put him in a soccer team when he's three with all those little kids that play soccer that are 3 or 4 years old. I want to do that so that's what I'm thinking I want a better life for him than you know, we had (Subject 10).

Play with him, going out with him, everywhere, be happy (Subject 11).

One of the women had fantasies that the child would always be there for her so she would not be lonely; "you'll have someone there, no matter what. You'll have someone there in case you feel lonely or something" (Subject 15). She lived with parents and husband.

A more realistic fantasy by one woman was how things might be when she had her baby.

A typical day, I will be tired, the baby will be by my side, maybe crying, I would give him a bottle, and try to take care of him. I know it's going to be a lot of pain or whatever but right now I don't want to find out what my baby's sex is going to be. I want to be surprised (Subject 9). 


\section{Unplanned Pregnancy/Acceptance}

All but one of the women stated that the pregnancy was a surprise but most were excited later. "It wasn't in my plans, but now that I am, I'm really happy because I'll be a mother and that's a really good feeling" (Subject 14) and "It was probably a surprise because it wasn't planned, you know, but once you find out, you're happy about it" (Subject 15). Another felt she should have waited, but took it for want it was; "I think maybe I could have waited, but things happen" (Subject 7-17). The one woman that stated she had planned the pregnancy had been married only a short time. "I got married on the fourteenth of November and we, I wanted a baby" (Subject 10).

The women expressed their attachment in similar ways. While most were not planning the pregnancy, they were accepting the changes in their lives and becoming attached to the idea of being pregnant and to their fetus. Only one painted her dreams in a more negative way, giving the idea that she knew the hardships ahead; I "will be tired"; "baby may be crying"; and" be a lot of pain" (Subject 9). This woman's mother initially had been very disappointed about the pregnancy and the father of the baby was not involved.

\section{Commitment}

Commitment is about making the decision to mother, giving up the concept of self, accepting the need to sacrifice, and responsibility for the child (Nelson, 2003; Mercer, 2006). Commitment involves pledging oneself to the act of mothering (Lydon, 1996).

Themes that emerged related to commitment were responsibility; sacrifice, changes made, missing out; and moving forward.

\section{Responsibility}

The majority of the women recognized that having a baby would be a big responsibility. One expressed that "I'm finally gonna know what responsibility is" (Subject 3). They thought that they now would have to do things on their own without help from their family; "Having to do things without my parents. Like doing a lot of things on my own" (Subject 6) and "I'm going to have to 
mature more, find a job" (Subject 6). One woman had been encouraged by family to have an abortion but she made the decision to have her baby; "if I took the responsibility to have my baby, I'll take the responsibility to be strong and be there" (Subject 9). The baby's dad was not involved and had been deported to Mexico. Being responsible meant what they had been taught by their families;

My grandmom used to say, always try to take care of your baby, treat her right, be responsible, and be loving, caring mother (Subject 4).

They were beginning to recognize that the responsibility of the baby took caring for the child night and day, "I think it will be like spending the whole day with her, every time she cries goes see what's wrong with her" (Subject 4); and "you know you have a bigger responsibility and that you have to take care of your kids" (Subject 15).

One of the women expressed that she needed to get ready for the responsibility because the time was coming and it wasn't just about the care and love;

Well there's just that responsibility there you know because the date is gonna come, the due date and right now we're barely starting on getting stuff for the baby; like the crib, like stuff for the baby. Be responsible. It's just getting ready for the baby basically now because and eating and taking care of myself

and being ok with myself because now it's not just me, it's my baby that's gonna be on the way. Just bring the baby here into existence is not going to need just the care and the love, and the looking out for it, it's going to need the diapers, it's going to need all of that so it's going to be a responsibility (Subject 20).

Others talked about what they would need to do to care for their baby; "try to be there for her every time, every night, every day. Take care of her whenever she's sick, feed her;" (Subject 14) and 'I try to get up early and feed the baby, get him ready, take him to my aunts' and then I'll get ready to go to work... its gonna be a hand full" (Subject 14).

Sacrifices, Changes, Missing out

The changes and sacrifices that they have made since they learned they were pregnant were discussed; "It's a big change, but I think it's going to be ok" (Subject 3); "I don't feel like having as much fun as I used to" (Subject 11). Many had to make life style changes such as 
living arrangements and finding a job; "I'm gonna go by myself;" I'm planning to move out now and try to be stable and try not to go out anymore" (Subject 14). She lives with her aunt and is not married. One of the couples was living with her mother, sisters, and brothers, and decided they needed to move since she was pregnant; "We decided to move out because we were living there with her, because we understand that we need our space too" (Subject 20).

Some of the women expressed that things might not be like they wanted, but that they would make the sacrifice and changes for the baby;

I think it's gonna change my life because at some point you could be immature but you have to try to be mature cause it's like not only you, it's like now with how the economy's going I'm gonna have to leave my baby with someone and go to work so I can give him or her whatever she wants, you know (Subject 15).

I think about being a mother. It might be kind of hard especially since I'm young. I'm 18 and I want to finish school and everything but at the same time it's something that's gonna change your life (Subject 15).

Having to do things without my parents. Like doing a lot of things on my own. I'm Going to have to mature more, find a job (Subject 6).

Having that thing that drives you to do that extra mile in everything that you do and I mean just, you know, being that example for that baby because everything that he's going to learn is going to be observing myself and my husband (Subject 20).

One of the women made sacrifices in her relationship with her family, especially when she decided not to have an abortion. She made the commitment but also made the sacrifice;

I understand now that everything is going and I took the position that I'm having my baby, she had to respect my decision and now she's [her mom] looking forward to my baby too (Subject 9).

Others made sacrifices and talked about missing out by not participating in sports or other activities with their friends;

I'm a really athletic person and so at church...I can't play volleyball and I miss that, so I can't do other activities that I used to do, but it's ok. I like playing around with my sister a lot, and now it's like don't touch me... it might hurt me, my baby (Subject 10).

I used to run, I used to do exercise; now I can't do the same things, but I'm happy. I'm not going to do the same things like I used to (Subject 11).

It feels great; it's great because it changed a lot of things. I used to go out a lot with 
my friends, but now I don't. I want to be with him [baby]; I don't want to left him by himself (Subject 17).

I did much more stuff before. Go out with my friends, time with them like you are pregnant and you can't do a lot of things....(Subject 6).

[Used to] spend too much time with others, like my friends but spending time with him, stop working for a while so I can take care of him until he's older (Subject 11).

I do the same thing. I go to school, I go to work. Except, well I used to have fun a lot. Like go out. It's not the same. It's going to be a big change but I think it's going to be ok (Subject 3).

\section{Move on/Go forward}

The majority of the women expressed that although the pregnancy was not planned; they

were happy and were making plans for a better time.

I think about holding it and how happy it's going to make me every time I look at it no matter what comes my way. I will be able to face anything that comes. I think it will make a big change because to love a stranger from one day to another and decide what's good for them without him or her being able to tell me anything about it (Subject 4).

I look forward to never give up and be there for my baby. I want to be there for my boy, my baby...I want to be there to tell him everything is gonna work out, I want to be there, the strongest one (Subject 9).

It wasn't in my plans, but now that I am, I'm really happy because I'll be a mother And that's a really good feeling (Subject 14).

I'm just trying to make a point here for all those women that don't have the support from their baby's daddy or if they do, just look forward to their life, a better life, you never know who's gonna be looking up to you and it could be a little kid, you'll feel so much wonderful (Subject 9).

The women were making plans and looking forward to what they wanted for themselves and their

baby even when others had concerns.

There's concerns right now about my mom that I will give up, that l'll say, mom you know what, I just can't do it, I'm leaving...I don't think I would have the heart to do that to my baby. I don't know how they would have the heart to leave their baby that is just born, abort a baby that is growing in your stomach that already feel you and kicked you and everything (Subject 9).

I'm gonna have the baby and then I wanna go back to school to a university and finish school. I don't know what l'll major in, like being a dentist or a nurse or something but just go to school and finish and move out and have our own house (Subject 15). 
It's just going to be looking out for the best of my family, and trying to get settled, and get a good job you know or something to give it all the needs that it needs. I'm seeing an overview of how I think it will go different for my family to actually be there and know what's going on around my family. Particularly be more involved in their activities.

We want to have a safe place for him mentally and physically and we really just want to make a difference. Not having a baby and bring it to violence or bring it to, you know, a not so good environment. So we're trying, the church helps out a lot. We talk to our pastors a lot also, and we just want to do something that makes the difference (Subject 20).

Once the women made the decision to be a mother, they began to accept the sacrifices and make changes in their lives to provide for the pregnancy and their baby. They had plans for the future although most were unable to verbalize how they would accomplish these plans.

\section{Preparation}

The mother needs to make preparation for her new role and involves the physical and psychological preparation for pregnancy and the child (Rubin, 1984). Her life experiences and help from others are a part of how the woman prepares for this experience. Themes for the construct of preparation were information and advice, being a mom, and classes.

Information and Advice

The majority of the women expressed that they received advice from family. Their mothers, aunts, and sisters provided advice about what they should be doing during their pregnancy and how to care for their baby.

Like when I'm eating junk food, like to eat healthy, take care of myself, till I have my baby. Mom talks mainly about having good nutrition (Subject 6).

[Her mom] eat well and stay active. You know, make sure I get my greens (Subject 10) I have to eat a lot of fruit, lots of vegetables, not to drink too much soda (Subject 11).

Eat right, take my vitamins, and mostly take care of myself, watch it whenever you walk, don't fall down (Subject 14).

[From mom] Like not to carry heavy stuff, or not to move real fast, there's a lot but I don't remember. Fruits, vegetables, don't eat a lot of stuff like McDonald's hamburgers or anything (Subject 17).

Tell me not to get mad (Subject 17). 
[mom] If I cry, feel sad, or mad, that's gonna affect my baby (Subject 9).

[mom] When something hurts, I ask her if it's normal or not or when I feel sick, what can I take? She's always there, like, do you want something to eat (Subject 20)?

They're just [family] orientating me you're going to be feeling this or it's ok. Or like, don't do this or don't that, just orient you through the pregnancy (Subject 10).

Really, really tired I guess, you know. My sister tells me the baby wakes up every 3 hours to eat, sometimes she doesn't get any sleep (Subject 10).

I'm learning [from mom] about that since I don't know how [feeding and diapering]. She tells me like if they're not hungry don't give them the bottle. Make sure you

change them like when they do the restroom and everything. To be checking with him, not to carry him too much and let him sleep and let other people get him too so he won't be like with just me.

She tries to explain to me, how to shower baby, or diapering, or breastfeeding, and the main things about it (Subject 4).

She told me that she's not going to help me that much because I have to learn. I have to learn, I have to take care of him (Subject 11).

[From aunt] showering her, feeding her, changing her diaper. This is the right way to put a diaper on, and if you're gonna breast feed, do it like this, this kind of stuff (Subject 14).

Two women shared that their mothers told them to ask their physician about a problem

they were having. One asked about what to do when she was having nausea, and the other said she would call the 1-800-hotline if it was something she thought was abnormal, but otherwise she would ask her mom (Subject 20).

Being a Mom

When describing how they would be as a mother and what they would be doing, some had difficulty knowing what it would be like while others had helped raise brothers and sisters and nephews and nieces. They felt this helped them prepare for being a mother.

I've never been involved with no kind of babies. I'm the youngest out of the whole family so she [her mom] tries to explain it to me (Subject 4).

I have a lot of practice. I have 7 or 8 nieces or nephews (Subject 10).

Spending time with my baby. Hard to think about what that will be like (Subject 11). 
When she [aunt] had her baby, I was helping her so I kind of know a little (Subject 14).

I don't know; playing with him, watching movies with him. I don't know.

Take him out to the park, I don't know..uh that he's clean all day and that, I don't know....(Subject 17).

I don't know everything but maybe I need some help when my baby's sick because that's probably one of the things I don't know what to do. I'll panic, but I know my mom's gonna be over there (Subject 10).

Like a good mom, that's what everyone wants to be. Always being there for their kid, like giving them every, well you want to give them, I think everyone

wants to give their kids everything they want. Stuff like that. You feel you're going the right direction (Subject 15).

I'm thinking about the environment where I'm going to be having my baby around with the influences around, rules (Subject 20).

Like with my mom with my two youngest sisters, like my three youngest brothers, they were like one right after the other so I would help my mom out (Subject 20).

I'm a woman that really wanted to be a mom. I considered myself, no one is really prepared to be a mother, but l'm prepared for what the consequences that are coming up.

I have to learn how to put these two things together, taking care of my baby and my husband too (Subject 10).

\section{Classes}

None of the women had definite plans to attend childbirth preparation classes or parenting classes. Two of the women had taken parenting classes in school. If they had considered taking classes, they did not know where to find them or they were not financially able to consider them.

No classes. I'll just try to go with what my mom raised me like (Subject 4).

I have heard about some friends that have heard about some places we could go, but not really (Subject 20).

I've been thinking about that, but l've been trying to get information about that too (Subject 10).

I would be glad to take um, but like I said, I was born in Mexico and so it's a lot of money. So what l'm thinking is to just have the money to buy pampers, milk, and that kind of stuff (Subject 9). 
I had classes in high school (Subject 11).

[classes in school] They showed us different kinds of birth control and they showed us a lot of stuff, and they ask us why we got pregnant or do we know. Questions like that so everybody in that class can know (Subject 15).

Their life experiences provided their preparation, with their mom, sister, or aunt providing advice.

Classes generally were not considered an option. They seemed to not consider it a necessity.

Culture, Family, Friends, and Partner

Description of culture was in terms of the beliefs, attitudes, and taboos that influence the process of mothering for the Mexican origin woman. Her family, friends, and partner are part of the environment in which the woman interacts and takes on the role of mothering. The women described their family's reactions to the pregnancy and from that, their beliefs and attitudes came forth.

\section{Culture}

Some of the women talked about how their Mexican culture helped them to be strong, but others said that their culture had provided challenges for them. They were not married, and they had experienced disappointment from their families.

Families, when you're immigrants, or come to the United States without papers, we look forward to a secure job and that's stressful and that's something that gets you down (Subject 9).

Seeing my family get disappointed, you know how in Spanish culture, they look forward to marriage couple, and virginity on Spanish girls is the main thing going on (Subject 9).

They wanted us to do, get married and all that, but there was not enough time [he was in army and sent to Iraq] (Subject 6).

Two of the women wanted to be different from her Hispanic culture in how she raised her children.

She [her mother] did a lot of things for us because when dad left us, so he went back to Mexico and she started working for us. She got two jobs, and then she... when we went with my dad they didn't want us to, they wanted us to have everything, that that's what she kept on doing. [she was expressing how this might not be good to give everything to the children] (Subject 17) 
Well, I want to be there in every activity with the baby, more like a friend. Be a parent, you know, but be into their activities... and I think every stage is important. and l've also learned from my dad's and mom's experience what kind of not to do with my kids because that's one thing with our Hispanic culture, that they really don't have open relationship with the kids, they just have the duty to bring the food to the house, have a roof over their head, but really like being, to know your kids,

they're kind of like, I don't know how to explain it.. to be more involved in their activities, to get to know what they're doing (Subject 20).

Family, Friends, and Partner

The family provided support, advice, and sometimes showed disappointment. All but two had their partner's support at varying levels. Disappointment was shown by their mother and by their partners.

\section{Disappointment}

I was happy at first, it didn't work out the way I wanted it to. The way my family reacted and the way my boyfriend reacted. Well, he I mean well he didn't want to have a baby, you know, but after, I guess he thought. You know, the fact that he was leaving for Iraq he told me. I didn't like that, it meant I had

to go through my pregnancy by myself. They wanted us to get married and all that, but there was not enough time (Subject 6).

They all got disappointed. They thought I was just going to not tell nobody, just get married right away, so I could cover stuff up (Subject 9).

My mom told me to abort the baby because she wasn't sure he [father of baby] was going to come back and take care of the baby (Subject 9 ).

At the beginning, my mom didn't want to even take a look at me and she was disappointed (Subject 9).

At first she was mad, but then she was like, she thought about it [laughing] (Subject 17).

She was kind of upset, she was like you should have tried to wait, but I think like every parent, they get over it, so she got over it (Subject 15).

Even though she didn't really want, she doesn't really want us to get pregnant or married at this age, she wants us to go to school and everything you knows, she's a good mother (Subject 10).

I was scared to tell my parents [they lived in Mexico] (Subject 3). 
Even though they expressed disappointment, all had the support of family and some were very excited. Some talked about the financial support their family would provide.

\section{Support}

I know my family will be there saying oh, he's beautiful or he's ugly or whatever.. but still it's your family, so they play around like that (Subject 9).

My mom didn't ask me much about it when I first told her as she does now, but she's happy (Subject 11).

I'm going to have all my family there, my aunts, my cousins, my grandma, my mom (Subject 11).

My husband's really excited (Subject 10).

They weren't mad, they were happy, too (Subject 11).

He's [partner] really happy (Subject 11).

There were three people, my mother, my aunt, and my grandmother. My grandmother was very loving; my mom was the responsible, and my aunt was the strict one; like you have to go to school, you have to do this (Subject 14).

We do talk about [the baby], my mom's like anxious like me. I want to see how she looks [laughing and excited] (Subject 14).

Well, he was like the first one so he was always telling me to make sure, you know, because he wanted to make sure. He wanted it to be a for sure (Subject 15).

Family members offered advice as way to offer support.

\section{Advice}

He's always telling don't do this, don't do that, don't eat this, he's more worried (Subject 17).

She's just like right there. She's always helping me with everything when I want to do something. She's like be careful, and she's like very careful with me and everything (Subject 15).

When I'm with my family, and we talk about how the babies are doing because I have other cousins that are pregnant (Subject 15).

My family was very supportive. My mom and my grandmum are the ones that tell me to take care of myself, not to have another one so soon (Subject 20).

He's always like are you ok... do you need something. He supports me how the baby is going to have his education and all also as he's growing up (Subject 20). 
They will definitely help me. They will give me advice, maybe we ask for advice, and I know for sure that if I'm going through economic problems, they will be there too to help me out (Subject 9).

We're living with my mom or she's living with us...we're living with each other to help each other out to save up money (Subject 10).

Even if the pregnancy had put a strain on their relationship with their family, they all expressed that they knew they could count on them for support.

\section{Emergent themes}

Three themes emerged from the participants' conversations; strong woman, determination to succeed, and a good mother. These themes add to the data already presented.

\section{Strong woman}

The woman is the center of the conceptual model, but the woman being a strong woman emerged as an emergent theme. Several expressed the need to be strong as a mother and provide for their baby. The women talked about their mothers as being strong or about how they would need to be strong to help their child.

I would want to be like my mom or I want to be as strong as my mom. I did it! I respect my mom, she supported 4 kids by herself. She never gave up and that's what I look forward to, never give up and be there for my baby (Subject 9).

She's a strong woman [her mom] and she's always dealt with her problems and never looked for you know. She's just a brave, strong woman (Subject 10).

Mainly she's [her mom] giving me, forcing me to be strong. So my mom is being there right now to make me strong. Another words, I have someone to look forward to and to be strong (Subject 9).

Any woman is gonna show strong and they don't wanna be seeing themselves, make themselves seen by other people that they're like not strong. They don't want to show their feelings because sometimes people get hurt that way (Subject 9).

They know that if I took the responsibility to have my baby, I'll take the responsibility to be strong and be there. And if it's working two times a day, will be something I have to do for my baby (Subject 9).

Well, I just imagine it like it being a little bit harder, but I feel I could be strong and everything that's hard make it easy for me and the baby (Subject 15). 
Having that thing that drives you to do that extra mile in everything that you do (Subject 20).

She did a lot of things for us because when dad left us, so he went back to Mexico and she started working for us. She got two jobs, and then she....they wanted us to have Everything, and that's what she kept on doing (Subject 17).

\section{Determination to Succeed}

Although the women used the word determined, the tones of conversation were always expressed strongly in that they accepted their challenges and were determined to make a better life for their baby. "Protect him and offer him the best and get him an education when he grows up (Subject 6)," "you can do it, make it (Subject 9)," and "I want to be there in every activity with the baby (Subject 20)." One of the women gave advice to other women to just keep going and looking forward to a better life.

I'm just trying to make a point here for all those women that don't have the support from their baby's daddy or if they do, just look forward to their life, a better life, you never know who's gonna be looking up to you and it could be a little kid, you'll feel so much wonderful (Subject 9).

They knew they had to prepare themselves for a new life and make new plans.

I have to prepare myself because I'm a single mother, so uh I have to get used to it because it is kind of hard. I had a job so I had that. I'm planning to move out now [her aunt's] (Subject 14).

Others were determined to continue their education so they would be able to satisfy their mother's dreams and provide for their children.

"My mom and my husband are very supportive of me going back to school once I have my baby" (Subject 10); "I'm going to go back soon [school] (Subject 10)," and "I'm still looking forward to getting my college even though I'm pregnant (Subject 9)."

I'm gonna try to uh well I'm gonna have the baby and then I wanna go back to school a university and finish school. I don't know what l'll major in like being a dentist or a nurse or something, but just go to school and finish and move out and have our own house (Subject 15).

She [her mom] wanted me to finish school, but I think I'm gonna finish...(Subject 17) 
I'm gonna take care of him, and then I'm gonna find a baby sitter and then l'll go back to school and become a medical assistant (Subject 3).

At first I'm gonna struggle, but Im gonna get through it (Subject 3).

One women wanted things to be different from how her parents raised her.

I'm seeing an overview of how I think it will go different for my family to actually be there and know what's going on around my family. Practically be more involved in their activities. We really want to have him in a place to have a safe place for him mentally and physically and we really just want to make a difference. Not having a baby and bring it to violence or bring it to, you know, a not so good environment. So we're trying, the church helps out a lot. We talk to our pastors a lot also, and we just want to do something that makes the difference (Subject 20).

The women were expressive in wanting more for their life although they weren't always sure how to accomplish their goals for a better life.

\section{Good Mother}

The women stated they wanted to be a "good mother", "A really good mother. One that understands the baby when he grows up knows what to expect" (Subject 11), and a good mother was one that was like their mom. Some were not sure how it would to be a mother; "I don't know, I think I will have to live the experience (Subject 4)." For many, their mothers were important to them and provided them with a role model for being a mother.

She [her mother] tells me it's beautiful when you're having a baby. Of course it's more beautiful when you're married and have your husband there to support you, but for any reason that doesn't happen, you have someone to look forward to (Subject 9).

My mom's been a really good role model to us and l've got a lot of; you know, examples from her and advice (Subject 10).

She a good mother, you know she's fought all these years being here in the United States, she speaks English really well, but she's given us really good example.

Go to church, that is the really good example she's given us. She's always maintained us there (Subject 10).

Like doing things my mom did for me. Because they need time, they need like their respect too, just like we do, and we need to take care of him, take care of my baby (Subject 11). 
One of the women expressed her desire to do things differently than how she had been raised, to be more involved with her children.

Well, I want to be there in every activity with the baby, more like a friend. $\mathrm{Be}$ a parent, you know, but be into their activities and I think every stage is important. I've learned from my dad's and mom's experience what kind of not to do with my kids (Subject 20).

Being a good mother is

To tell him what's right and not to let him what he wants or buy him what he wants because that's bad for him and not to let him be so aggressive with other kids (Subject 17).

Like a good mom, that's what everyone wants to be [laughing]. Always being there for their kid, like giving them everything, well you want to give them, think everyone wants to give their kids everything they want. Stuff like that. You feel you're going the right direction (Subject 15).

Hopefully like my mom, because l've always liked the way she raised us you know. I admire her so much (Subject 10).

The women wanted to be a good mother. They had definite ideas about what a good mother would be like.

\section{Discussion}

Telephone interviews were planned for the data collection based on the literature that Hispanics tend to provide information that is more valid by telephone (Gilliss, Lee, et al, 2001; Harris, Ahluwalia, et al, 2003; Auerswald, Greene, et al, 2004). This method was not effective for the women recruited for this study. The ten Mexican origin women interviewed provided stronger data face to face with the researcher and cues for further questioning were easier to read.

The women were interviewed using the model as the basis for the interview questions and the analysis. Themes were permitted to emerge from the transcribed interviews and additional themes emerged from the data that did not fit within the model. 


\section{Attachment}

At the time of the interviews, all of the women had moved through the acceptance phase of the pregnancy (Rubin, 1984). The women described attachment first by stating concerns they had about their pregnancy. It was a planned pregnancy for only one woman. Descriptions of initial worries/uncertainties related to unplanned pregnancy were similar to the findings of Miller (2005) who indicated they were concerned about getting it right and scared because they were still in high school. There was the conflict between trying to maintain their sense of self while meeting the expectations of others.

All women described fantasies and dreams about the baby which corresponds to Rubin's (1967a, 1967b) descriptions of Fantasy and Introjection-Projection-Rejection of "how it will be for me". They described the baby as being an older child and often of a certain sex. The sex was identified by the way they saw the child dressed. They had daydreams of playing with the child, soccer or answering their questions. When expressing joy, the women were finding a way for the baby to "fit" into their life and for the mother to take on the new role (Rubin, 1967a, 1967b). Fantasy and dreams for the participants were significant in development of the mothering role echoing the work of Rubin (1984) and Lederman (1984, 1996).

\section{Commitment}

The majority of the women displayed commitment to the pregnancy and to the child. As Nelson (2003) and Mercer (2006) described, the women realized the sacrifices they must make and the responsibility of having a child. In an earlier study, Lyndon (1996) noted that commitment involved pledging oneself to the act of mothering. He noted that commitment was grounded in their values, beliefs, and identities. The participants in this study said that their mothers and grandmothers taught them responsibility. They realized they would need to not only love and care for the child but provide material things as well, like diapers, clothes, and food.

Commitment connotes energy and action (Lyndon, 1996). The women described the adjustments in their daily activities, their plans for the future, and their eating habits. Lederman 
(1996) identified pregnancy as a transition between two life styles, "the women without-child" to a "woman-with-child". The woman grows or moves forward, just as the participants in this study expressed when sharing their plans for her life with the child. The women shared that they had to stop going out as much and to watch what they ate. The women discarded old ways and there was a reevaluation of self and significant others to make plans for the baby as Lederman (1996) described.

Acceptance of the pregnancy, although not planned by most of the participants, was described by the participants when they shared how they had decided to continue their pregnancy, tell their parents and partner and begin to formulate a relationship with their child. They began to see themselves in the role as a parent. Colman and Colman (1973) noted that pregnancy is neither "static or brief, but one full of change and enrichment," (p.1) just as the participants used their pregnancy to organize their life and decide how they would participate in their child's life.

Preparation

During pregnancy, the woman prepares for her new role as a mother both physically and psychologically (Rubin, 1984). The participants sought advice and information from their mothers, aunts, and sisters as described by Colman and Colman (1973) who suggest that women benefit from being with other women to discuss concerns and problems. Consultation of medical personnel occurred only if they thought it was "abnormal" or if their mother suggested they ask.

When the participants described what they would be doing as a mom after the baby was born, some could not imagine it. Those who had cared for siblings or nieces and nephews were able to verbalize some of the activities they would be doing. They felt that it would be hard, but they would be able to handle it. Participants' descriptions were similar to Miller's (2005) reflections on this experience that often showed their expectations did not match the real experience of being a mother. 
In response to the question about plans to take formal prenatal and parenting classes, they did were not consider taking them. Although some thought it might be a good idea, they could not afford the expense. Murphy (2008) suggested that women do not consider attending prenatal classes because of factors such as the timing of classes, already knowing the information, and costing too much. Svensson, Barclay, and Cooke $(2006,2007)$ noted that health care providers perceive the woman's needs differently than they do, therefore they do not meet the participants' learning needs. Most of the participants felt they did not need to attend a class because they would do as their mother had done. Prenatal classes prepare the couple more for labor (Murphy, 2008) and the participants did not think this type of preparation was necessary; it would happen and they would get through it with help from their mother, sisters, or aunt.

\section{Culture, Family, Friends, and Partner}

All the participants of this study had parents who were born in Mexico. All but two of the women were also born in Mexico and only one spoke English exclusively at home. The participants considered being a Mexican as either a strong asset or a challenge. Their conflict was reflected by family expectations to behave a certain way, and when they did not, their families were disappointed. Berry (2002) suggested that acculturation affects the psychology of the individual. The external culture and the changing culture influenced them. Measurement of acculturation occurred informally. All but one of the women spoke either Spanish or English/Spanish at home. All but two were born in Mexico. Marín and Marín (1991) suggest that language use is one of the changes produced by acculturation and is the easiest to measure. It has become a reliable shorthand measure for evaluating acculturation.

Emotional and physical support received by participants was consistent with the literature (Negy, 1993; Jones \& Bond, 1999; Balcazar, et al, 2001; Langorá \& Gonzalez, 2003). The women described how they were pampered, told to eat right, rest, and not to worry or get upset. Participants expressed how their family would always be there for them, even if they initially were upset with them for being pregnant. Researchers have found that family is a key component of 
their support (Becerra \& de Anda, 1984; Berry, 1999; Sagrestano, et al, 1999; Balcazar, et al, 2001; Domian, 2001). Only one of the participants had planned her pregnancy. The support from their mothers or aunts was strong; it was their key support. Two of the women who had a strong relationship with the father of the baby discussed how they relied on them as well as their mother. Sherwen (1987) suggested that pregnancy is a family affair. The words of the participants in this study described how everyone was preparing for the baby.

The study by Becerra and de Anda (1984) noted that English-speaking Mexican American girls were more at risk for unwanted pregnancies and births outside of marriage than Spanish-speaking Mexican Americans and white non-Hispanics and may be affected by acculturation. Participants in this study had been in the United States 6-20 years and spoke English/Spanish and all of their mothers had been born in Mexico. All but one of the women had an unplanned pregnancy. Miller (2005) suggested that there is a struggle to try to maintain a sense of self versus what their culture expects of them. Several of the participants discussed the conflict of their pregnancy and the expectations their mothers had about marriage and finishing their education. As Miller (2005) noted, the participants presented a picture of acceptable preparation for motherhood, but they acknowledged some inconsistencies of support.

\section{Emergent themes}

The emergent themes of strong woman, determination to succeed, and good mother are cyclic in nature and are interdependent. The woman's personality characteristics of ego strength and nurturance are considered important in predicting pregnancy outcomes (Sherekansky \& Yarrow, 1973; Sank, 1991). The Mexican origin participants expressed the need to be strong emotionally, to be like their mother, who is strong. They felt like their inner strength and resilience would help them as a mother. Dingley, Roux, and Bush (2000) noted in a concept analysis of inner strength that the literature presented the concept as "a quality of leadership, a component of spirituality, a facet of quality of life, a contributor toward overcoming problems, a dimension of empowerment, and a component of psychologic health and healing" (p.34). 
Callister (2001) noted that health care should build on the strengths of these women instead of assuming and emphasizing their deficits.

The Mexican origin women's determination to succeed and make a better life for themselves and their child emerged to show how they wanted to provide for their children, but also to succeed themselves. They wanted to return to school, get a better job, and have a better life. Deci, Vallerand, Pelletier, and Ryan (1991) suggest that when a behavior is self-determined, the regulating process is choice but if controlled, the process is compliance or defiance. The participants expressed the desire to have control over their lives. The women cherished motherhood, but it was not life defining, which is consistent with findings from other studies with Mexican origin women (Amaro, 1988; Guendelman, Malin, Herr-Harthorn, \& Varlas, 2001; Chun \& Akutsu, 2002). The participants recognized their mother's strength, but several expressed a desire to express their strength differently for themselves and their family. Some of the participants were going to be just like their mothers and raise their children the same, but others wanted to change from how their parents raised them. They wanted to be able to be a part of their child's life and not just provide food and a place to live.

Expression of the theme of "good mother" was expressed in the words of the participants by stating they wanted to be like their mothers. Being a "good mother" seemed to be tied with the determination to succeed and being a strong woman. Miller's (2005) study noted the participants felt that a "good mother" was one that had a safe birth. For the participants' in this study, being a "good mother" appeared to have a deeper meaning where they would provide for their child over the years. Many of them suggested they would no longer be going out as much, but staying close to their baby and making a difference in their lives, yet they also realized they wanted to make a life better for themselves.

These three emergent themes come back to the core of the model, are cyclic, and separation may not be possible within this group of women. The women wanted to persevere and maintain their own dignity while giving their child the love and guidance they would need. The 
woman at the core is now depicted as strong and is determined to succeed in the process of becoming a mother.

\section{Limitations}

The findings of this study are not generalizable which is appropriate with qualitative research undertaken to understand the experience of this unique group of women. This study used a small sample conducted at one geographic setting. The criteria for participation in the study limited the study to a small group of Mexican origin women, 18-23 years of age. All of the women that participated attended the same community clinic, which provides care to a predominately Hispanic population. Mexican origin women of generationally and socioeconomically variations might experience the process of anticipating mothering differently. Langaná (2003) discussed the idea of biculturalism, the phenomenon that allows the women to maintain or reject culturally dictated practices and beliefs to fit their needs. All of the women spoke English well and although they shared their thoughts and feelings with the researcher in English, they may have been unable to express their emotions and thoughts as well as they might in their first language, Spanish. Mosenthatl (2001) suggests, "language molds a person's experiences, emotions, and thoughts before they are realized" (p307). Emotions are expressed in the first language learned as a child and the words and concepts may not exist or share the same meaning in a different language (Altarriba \& Santiago-Rivera, 1994). Each of the women had been in the United States for 6-20 years and been in school during that time.

\section{Conclusions}

The Mexican origin women who participated in this study presented a picture of anticipating mothering that is congruent with the model and the literature presented in chapters one and two. This study adds a greater emphasis on culture, family, and partner support that was very important to them. Their mothers were especially important to them. When discussing their mothers, the women wanted to be like them. However, in the case of two of the women, they wanted to do things differently from their parents and be more involved with their children. 
They felt that Hispanic families only felt it was important to provide food and shelter and not be a part of their child's life. If the partner was actively involved, his role was also important. Their friends were only mentioned as being important if they were pregnant or had children, but they did not seem to play a role in the process of anticipating motherhood for this group of women.

Being a strong woman gives these women the determination to succeed. However, the determination to succeed may actually provide them their strength. Both of these result in their desire to become a good mother. These three themes need to be explored further to understand if they should be incorporated in the model for this population. The women were all looking for a better life for themselves and their child. Since the population of the United States is changing to a larger population of Hispanics, and $48 \%$ of them are women, ways to capitalize and further study these themes must occur.

Implications for Nursing

The findings from this study suggest that nurses and other health care providers need to be aware of the cultural patterns of this group of women during their pregnancy. Nurses can benefit from the information shared by the women in this study. It is clear that most of the women were not planning a pregnancy at this time, and although it was hard and would continue to be hard, they were moving forward with plans using the support offered by families. The women in this study spoke about the importance of their families, their religion, and how there was some conflict between their mother's way of doing things and the way they planned to raise their children. The importance of their family, cultural values and desire to succeed provides opportunities to capitalize on these aspects to suggest changes in the way care and information is provided. As noted in Chapter 1, Berry (1999) suggested that Mexican American women viewed care by nurses as culturally appropriate when they respected family caring roles based on age and gender. The women respected the nurses' knowledge and being attentive and protective of their needs. As Callister (2001) suggested, health care providers need to capitalize on the strength of these women instead of their deficits as a way of providing care. Because of 
the importance of the other women in the expectant woman's life, suggestions for changes in formal prenatal classes as the primary mode of preparation for birth and parenting need to be considered. Services need to be in place to support and to enhance the existing resiliency (determination and strength) with this population and further their desire to be good mothers. By attending to the beliefs and needs of these women, they will be able to use their strength and determination to be a "good mother".

Recommendations for Additional Research

The findings of this study provide opportunities for further research using multiple methods, conducting comparisons of women of different ages, gestational age, and levels of acculturation. A longitudinal study of a group of women over the course of their pregnancy and after birth would give a clearer picture of the different phases of anticipating mothering and the influence on early mothering. This study would entail interviewing the same women at various times during their pregnancy and after delivery. There is a need for more research to understand how to capitalize on the women's strength and use this strength in the health care system of today using the "best of both worlds". This longitudinal study would provide information on the characteristics of the women as strong and determined to succeed as a "good mother", as a means of promoting better outcomes for their child. Focusing on the individual's strength may shift knowledge toward the idea of wellness orientation and healthy functioning instead of inadequacy and impairment (Dingley, Roux \& Bush, 2000; Callister 2001).

An additional area of research warrants further examination is the difference between immigrant and intergenerational (born in the United States) beliefs that affects the process of mothering. This includes family importance, acculturation, and differences in socio-cultural status. An acculturation tool was not a part of this study, but would be a key area of interest for further research.

In summary, it is clear that these women were excited about the idea of being a mother. Only one had planned a pregnancy at this time, others had experienced disappointment from 
important people in their lives. The women were making plans and were determined to succeed as a good mother. As the population of the United States changes and the role of the Hispanic population grows, health care providers need to begin understanding what role these women will have in caring for their families including assistance they need and want. A greater understanding of how mothering occurs with this group of women will help in planning programs to capitalize on their strength and determination to succeed. Only by attending to the beliefs and thoughts of the women can nurses hope to affect change and offer support to enhance the lives of the women and their children. 
APPENDIX A

PERMISSION FOR USE OF MODEL 


\section{University of Calfomis \\ Ban Francisco \\ UCSF \\ School of Nursing \\ Department of Family Health Care Nursing}

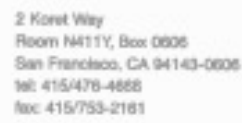

April 7, 2008

Susan M. Baxley, RNC, MS, PhD Candidate

$1114 \mathrm{~N}$. Windomere Avenue

Dallas, Texas 75208

Dear Ms. Baxley,

It is always exciting to see a rising scholar extend any theoretical statement that has been proposed. Your proposal and model of the process of anticipating mothering are very important in contributing to the theory of becoming a mother.

Your proposed dissertation research will contribute to the theory by illustrating how women in another culture negotiate the anticipation phase. Further, your research will help validate the usefulness of the theory across cultures.

I am very pleased to see that my work is contributing to your important research. Best wishes to you in this endeavor.

Sincerely,

Pamour 2 pucen

Ramona T. Mercer, PhD, FAAN

Professor Emeritus

1809 Ashton Avenue

Burlingame, CA 94010 
APPENDIX B

DEMOGRAPHIC FORM 


\section{Demographics}

1. Code

2. Date of contact

3. Interview date

4. Initials

5. Age

Date of birth

6. Gestational age Due date

7. County origin

8. How long lived in U. S.

9. Language spoken at home

10. Work School

11. Married

12. Live with?

13. Partner involved? 
APPENDIX C

DATA COLLECTION FLOW CHART 


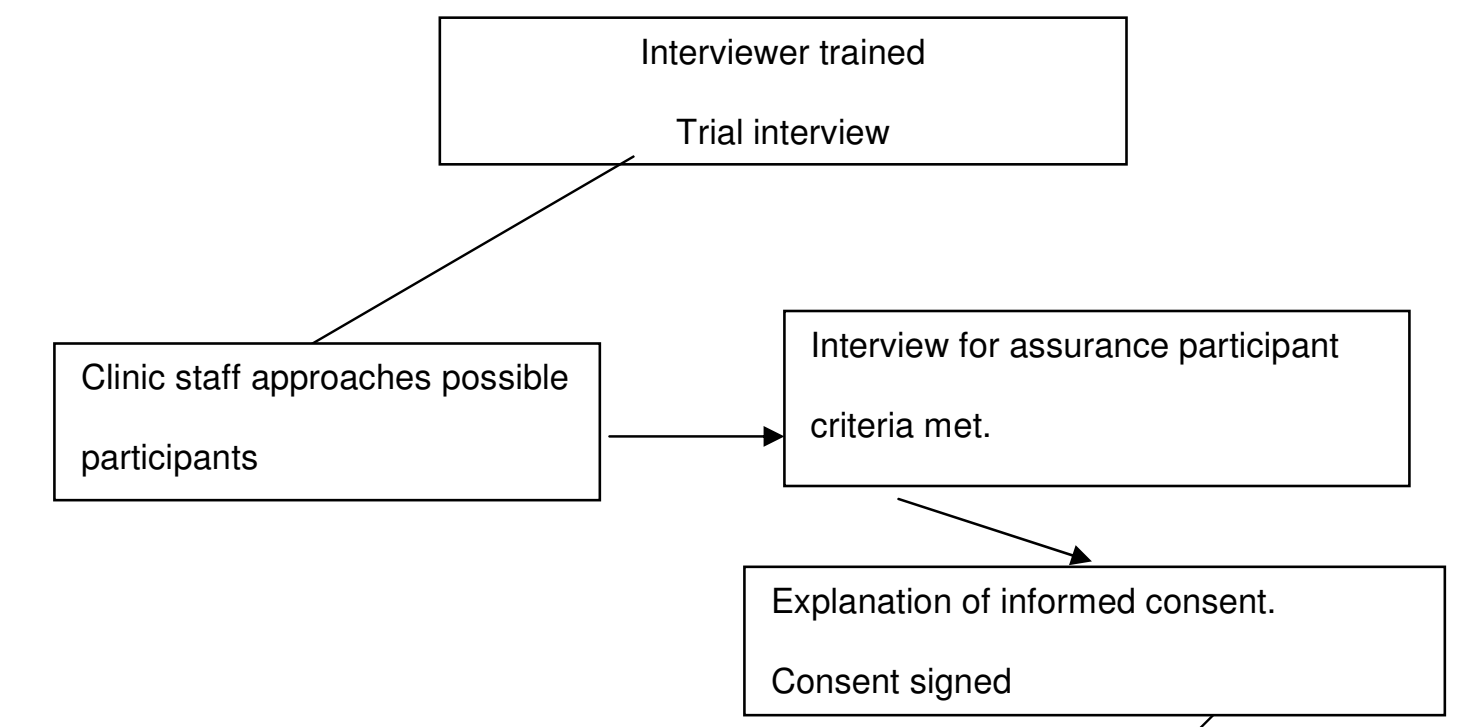

Telephone number obtained and time for telephone interview set or interview conducted. The time set for a later interview was based on the woman's gestational age.

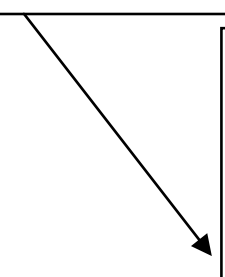

Telephone and face to face interview:

1. Began with re-explanation of study and verbal consent to participate.

2. Digital recording began.

3. Verbal consent to participate stated again and recorded agreement by participant.

4. Researcher started interview by asking demographic questions.

5. Grand tour questions asked, with follow-up questions.

6. The interview took approximately $20-40$ minutes

Interviews conducted by telephone had data reviewed at another prenatal visit. Interviews conducted face-to-face, clarified the information at the end of the interview.

She received a gift certificate for her time.

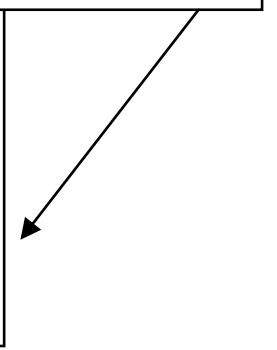




\section{APPENDIX D}

AUDIT TRAIL FOR DATA COLLECTION 
Audit Trail-Data Collection

N

\begin{tabular}{|c|c|c|c|c|c|c|c|c|c|c|}
\hline & $A$ & $B$ & $\mathrm{C}$ & $\mathrm{D}$ & $E$ & $\mathrm{~F}$ & $G$ & $\mathrm{H}$ & $\mathrm{I}$ & $\mathrm{J}$ \\
\hline 1 & code \# & $\begin{array}{l}\text { date of 1st } \\
\text { contact }\end{array}$ & $\begin{array}{c}\text { consent } \\
\text { signed }\end{array}$ & $\begin{array}{c}\text { interview } \\
\text { date }\end{array}$ & site & $\begin{array}{c}\text { phone/ } \\
\text { in-person }\end{array}$ & interviewer & confirmed content & $\begin{array}{l}\text { reasons not } \\
\text { done }\end{array}$ & Notes \\
\hline 2 & 1 & $10 / 31 / 2007$ & yes & none & site \#1 & & & & $\begin{array}{l}\text { didn't answer } \\
\text { phone }\end{array}$ & \\
\hline 3 & 2 & $11 / 15 / 2007$ & yes & none & site \#1 & & & & $\begin{array}{l}\text { didn't answer } \\
\text { phone }\end{array}$ & \\
\hline 4 & 3 & $2 / 5 / 2008$ & yes & 2/26/2008 & site \#2 & in person & researcher & $\begin{array}{l}\text { at time of interview/ } \\
\text { didn't want to review }\end{array}$ & & $\begin{array}{l}\text { Had boyfriend at } \\
\text { interview }\end{array}$ \\
\hline 5 & 4 & $2 / 19 / 2008$ & yes & 2/19/2008 & site \#2 & phone & $\begin{array}{c}\text { M.O. } \\
\text { interviewer/ } \\
\text { researcher }\end{array}$ & $\begin{array}{c}\text { reviewed at her next } \\
\text { visit }\end{array}$ & & \\
\hline 6 & 5 & 3/4/2008 & yes & & site \#2 & phone & & & $\begin{array}{l}\text { wanted to do in } \\
\text { Spanish }\end{array}$ & \\
\hline 7 & 6 & $3 / 5 / 2008$ & yes & $3 / 5 / 2008$ & site \#2 & in person & researcher & $\begin{array}{l}\text { at time of interview/ } \\
\text { didn't want to review }\end{array}$ & & $\begin{array}{l}\text { had girl friend with } \\
\text { her }\end{array}$ \\
\hline 8 & 8 & $3 / 14 / 2008$ & yes & & site \#2 & & researcher & & & $\begin{array}{l}\text { transferred to high } \\
\text { risk clinic }\end{array}$ \\
\hline 9 & 9 & $3 / 14 / 208$ & yes & $4 / 11 / 2008$ & site \#2 & in person & researcher & $\begin{array}{c}\text { at time of } \\
\text { interview/didn't want } \\
\text { to review }\end{array}$ & & \\
\hline 10 & 10 & 4/11/2008 & yes & $4 / 11 / 2008$ & site \#2 & in person & researcher & $\begin{array}{c}\text { at time of } \\
\text { interview/didn't want } \\
\text { to review }\end{array}$ & & \\
\hline
\end{tabular}




\begin{tabular}{|c|c|c|c|c|c|c|c|c|c|c|}
\hline & $\bar{A}$ & $B$ & $\mathrm{C}$ & $\mathrm{D}$ & $\bar{E}$ & $\mathrm{~F}$ & $\mathrm{G}$ & $\mathrm{H}$ & $\mathrm{I}$ & $\mathrm{J}$ \\
\hline 11 & code \# & $\begin{array}{c}\text { date of 1st } \\
\text { contact }\end{array}$ & $\begin{array}{l}\text { Consent } \\
\text { signed }\end{array}$ & \begin{tabular}{|c|} 
interview \\
date
\end{tabular} & site & $\begin{array}{c}\text { phone/ } \\
\text { in-person }\end{array}$ & interviewer & confirmed content & $\begin{array}{l}\text { reasons not } \\
\text { done }\end{array}$ & Notes \\
\hline 12 & 11 & 3/18/2008 & yes & $4 / 1 / 2008$ & site \#2 & in person & researcher & $\begin{array}{c}\text { at time of } \\
\text { interview/didn't want } \\
\text { to review }\end{array}$ & & \\
\hline 13 & 12 & 3/28/2008 & yes & & site \#2 & in person & researcher & & $\begin{array}{c}\text { didn't keep } \\
\text { clinic } \\
\text { appointments }\end{array}$ & \\
\hline 14 & 13 & 3/28/2008 & yes & & site \#2 & in person & researcher & & $\begin{array}{l}\text { conflict with } \\
\text { husband }\end{array}$ & $\begin{array}{l}\text { 7-9-08 She was } \\
\text { having conflict with } \\
\text { husband, didn't want } \\
\text { to do interview. Had } \\
\text { missed several } \\
\text { appointments at clinic }\end{array}$ \\
\hline 15 & 14 & 3/28/2008 & yes & $4 / 11 / 2008$ & site \#2 & in person & researcher & $\begin{array}{l}\text { at time of interview/ } \\
\text { didn't want to review }\end{array}$ & & \\
\hline 16 & 15 & $4 / 11 / 2008$ & yes & $4 / 11 / 2008$ & site \#2 & in person & researcher & $\begin{array}{l}\text { at time of interview/ } \\
\text { didn't want to review }\end{array}$ & & \\
\hline 17 & 16 & $3 / 14 / 2008$ & yes & & site \#2 & in person & researcher & & $\begin{array}{l}\text { didn't want to } \\
\text { partici-pate }\end{array}$ & \\
\hline 18 & $7 \& 17$ & $3 / 14 / 2008$ & yes & $5 / 16 / 2008$ & site \#2 & in person & researcher & $\begin{array}{c}\text { at time of } \\
\text { interview/didn't want } \\
\text { to review }\end{array}$ & & $\begin{array}{l}\text { on 4-15-08 did not } \\
\text { answer phone then } \\
\text { later done at clinic }\end{array}$ \\
\hline 19 & 18 & $5 / 23 / 2008$ & yes & & site \#2 & in person & researcher & & $\begin{array}{c}\text { didn't keep } \\
\text { clinic appoint- } \\
\text { ment }\end{array}$ & \\
\hline 20 & 19 & $5 / 23 / 2008$ & yes & & site \#2 & in person & researcher & & $\begin{array}{c}\text { didn't keep } \\
\text { clinic appoint- } \\
\text { ment }\end{array}$ & \\
\hline 21 & 20 & 6/11/2008 & yes & $6 / 11 / 2008$ & site \#2 & in person & researcher & $\begin{array}{l}\text { at time of interview/ } \\
\text { didn't want to review }\end{array}$ & & \\
\hline
\end{tabular}




\section{APPENDIX E}

INSTITUTIONAL REVIEW BOARD CONSENTS, APPROVAL LETTERS 


\section{APPROVED MHSIRB}

\section{METHODIST HEALTH SYSTEM \\ RESEARCH INFORMED CONSENT}

\section{Anticipating Mothering for the Mexican Origin Woman IRB\# 2007.00.416}

Principal Investigator: Tony Paterniti, $\mathrm{PhD}, \mathrm{CCHt}$, $\mathrm{RN}$; Co-Investigator: Susan $\mathrm{M}$. Baxley, RNC, MS, PhD candidate. Faculty Advisor: Mary Lou Bond, RN, PhD, FAAN

Are you in any other research studies? Yes $\underset{\text { Please initial your response }}{\text { No }}$

You have been invited to participate in a research study. Understanding this study's risks and benefits will allow you to make an informed judgment about whether to be part of it. This process is called informed consent.

This consent form may contain words that you do not understand. Please ask the study staff to explain any words or information that you do not clearly understand. You may take home an unsigned copy of this consent form to think about or discuss with family or friends before making your decision.

In this consent form, "you" always refers to the subject. If you are a legally authorized representative, please remember that "you" refers to the study subject.

\section{PURPOSE}

The purpose of this study is to explore the meaning of attachment, commitment, and preparation during pregnancy as an anticipatory phase of mothering for the Mexican origin woman giving birth in the United States.

You have been asked to participate in this study because you are pregnant with your first child, self-identified Mexican origin, between 18 and 22 years old, 25-30 weeks pregnant, and speak English. (Mexican origin: living in the United States, born in or tracing the background of your family to Mexico.)

\section{PROCEDURE:}

There are four times you will meet with the researcher. The 4 times are explained below.

1. You will have a clinic visit at the Golden Cross Clinic. At this time you will be told about the research and have the opportunity to ask questions about the research. If you agree to participate, you will sign this informed consent. Once you agree to be a part of the study, you will give the researcher your telephone number and suggest the best time for us to call you. We will call to set a time for the interview and then call the day before to confirm the interview. 


\section{APPROVED MHSIRB}

Preparation Date (July 13, 2007) Revision Date (October 10, 2007)

2. The second visit will be conducted on the telephone. A nurse of Mexican origin will conduct the interview with the researcher available for questions during the interview. The interviewer will ask you questions about your pregnancy and your baby. The phone call will be audio-taped and last approximately 30-60 minutes, as necessary for you to share your experience and thoughts.

3. The third visit will again be at the Golden Cross Clinic during a pre-arranged prenatal visit. You will review a summary of your interview and check it for accuracy. You may provide any additional information that you may choose to share at this time. (A \$25.00 gift certificate will be given to you at this time for your participation).

4. After the final review of all interviews by the researcher, you may receive a copy of a summary of the study if you would like. If you prefer to not meet, you may provide your address and the summary can be mailed to you.

There will be approximately 10-20 participants.

The total time to participate is approximately 2 hours for four visits with the interview taking the majority of your time. The total time for completion of the study should be no longer than 6 months to one year.

\section{$\underline{\text { RISKS }}$}

The possible risks and/or discomforts of your involvement include:

During the interview process, you may experience possible psychological discomfort while talking about your feelings. There is always a potential for loss of confidentiality. After the study, your consent and your interview will be kept separately and stored in a locked file cabinet in the Center for Nursing Research, The University of Texas at Arlington, School of Nursing.

\section{$\underline{\operatorname{cosT}}$}

There are no financial costs to you to participant in this study.

\section{PAYMENT FOR PARTICPATION AND/OR REIMBURSEMENT OF EXPENSES}

You will receive a \$25.00 gift card and Certificate of Appreciation during the third contact when we meet to review the summary of your interview. The card and certificate are given as a thank you for your time to participate in this study.

\section{COMPESENATION FOR INJURY}

Neither the investigators conducting this study nor Methodist Health System are able to offer financial compensation should you be harmed as a result of your participation in this research. 


\section{APPROVED MHSIRB}

Preparation Date (July 13, 2007)

Revision Date (October 10, 2007)

You should know that by signing this form, you are neither waiving any of your legal rights against nor releasing the principal investigator, sub investigator and Methodist Health System or any of their respective agents from liability for negligence with respect to the conduct of this study. For more information about claims, you may contact the Chairman of the Institutional Review Board of UTA at 817/272-1235 or Methodist Health Systems Institutional Review Board at 1441 N. Beckley, Dallas, Texas 75203.

\section{AUTHORIZATION TO USE AND DISCLOSE INFORMATION FOR RESEARCH PURPOSES}

This study will use the following health information or information from your research or medical record:

- Age

- Week of your pregnancy

- Number of pregnancy

- Ethnic origin

- Record of the phone call

- Record of study visit

- Demographic information

Every attempt will be made to see that your study results are kept confidential. A copy of the records from this study will be stored in the Center for Nursing Research at the School of Nursing. The University of Texas at Arlington. All audio tapes, consents and written records of your interview will be kept in a locked cabinet in the Center for Nursing Research for at least three (3) years after the end of this research and then destroyed by shredding and the tape destroyed by crasing the information. Once the study is complete, your telephone number and name will be shredded. The consent and interviews will be coded by number and stored separately.

Why will this information be used and/or given to others?

The results of this study may be published and/or presented at meetings without naming you as a subject. Although your rights and privacy will be maintained, the Secretary of the Department of Health and Human Services, the Methodist Health System Review Board, the UTA Institutional Review Board, and personnel particular to this research (individuals) have access to the study records. Your medical records will be kept completely confidential according to current legal requirements. They will not be revealed unless required by law, or as noted above. Your medical record will be reviewed by clinic personnel to screen for the inclusion criteria: age, stage of pregnancy, if first pregnancy, and of Mexican origin. The researcher and interviewer will not review your medical record. 


\section{APPROVED MHSIRB}

What if I decide not to give permission to use and give out my health information? By signing this consent form, you are giving permission to use and give out health information listed above for the purposes described above. If you refuse to give permission, you will not be able to be in this research.

May I review or copy the information obtained from me or created about me? You have the right to review and receive a copy of a summary of your interview, if you decide to be in this study and sign this permission form. Later, if you request, you may receive a copy of a summary of the final study.

May I withdraw or revoke (cancel) my permission?

This permission will be good until you have completed the interview and met to look over a copy of your interview to see if it is what you wanted to say. You may withdraw or take your permission to use your interview at any time. If you withdraw your permission, you will not be able to continue being in this study.

When you withdraw your permission, no new information will be gathered after that date. Information that has already been gathered may still be used. This would be done if it were necessary for the research to be reliable.

Is my health information protected after it has been given to others? If you give permission to give your identifiable health information to a person or business, the information may no longer be protected. There is a risk that your information will be released to others without your permission. Your personal information may be disclosed if required by law.

\section{QUESTIONS}

If you have any questions concerning your participation in this study or if at any time you feel you have experienced a research-related injury, contact:

Tony Paterniti PhD, CCHt., RN, Methodist Health Systems at 214-947-1562

Susan M. Baxley, RNC, MS, PhD candidate at 214-505-9161

Address: University of Texas at Arlington School of Nursing

P. O. Box 19407

Arlington, TX 76019-0407

Faculty advisor, Mary Lou Bond, RN, PhD at 817-272-1271

If you have any questions about your rights as a research subject, you may contact: Methodist Health Systems Institutional Review Board

Director: Phyllis Everage

1441 N. Beckley Avenuc

Dallas, Texas, 75203

214-947-2542

Methodist IRB is a group of people who perform independent review of research. 


\section{APPROVED}

MHSIRB

Preparation Date (July 13, 2007)

Revision Date (October 10, 2007)

If this consent form is read to the subject because the subject (or legally authorized representative) is unable to read the form, an impartial witness must be present for the consent and sign the following statement:

I confirm that the information in the consent form and any other written information was explained, and the subject was given the opportunity to ask questions (or the subject's legally authorized representative). The subject (or the subject's legally authorized representative) freely consented to participate in the research study.

Signature of Impartial Witness

Printed Name

Date

Note: This signature block cannot be used for translations into another language. A translated consent form, with the translation approved by the IRB, is necessary for enrolling subjects who do not speak English. 


\section{APPROVED MHSIRB}

Preparation Date (July 13, 2007)

Revision Date (October 10, 2007)

Do not sign this consent form unless you have had a chance to ask questions and have received satisfactory answers to all of your questions.

If you agree to participate in this study, you will receive a signed and dated copy of this consent form for your records.

\section{VOLUNTARY PARTICPATION AND WITHDRAWAL}

Participation in this study is voluntary. You may decide not to participate in this study or you may withdraw from this study at any time without penalty or loss of benefits to which you are otherwise entitled at this site.

Your participation in this study may be stopped at any time by the investigator or the University or Hospital without your consent because:

- the investigator thinks it is necessary for your health or safety;

- the sponsor has stopped the study; or

- administrative reasons require your withdrawal.

- you have your baby prior to being interviewed.

\section{CONSENT}

I have read the information in this consent form, (or it has been read to me). All my questions about the study and my participation have been answered. I freely consent to participate in the research study. I authorize the use and disclosure of my health information to the parties listed in the authorization section of this consent for the purposes described above. By signing this consent form I have not waived any of the legal rights which I otherwise would have as a subject in a research study.

\section{CONSENT SIGNATURE:}

\begin{tabular}{lll}
\hline Participant Signature & Printed Name & Date
\end{tabular}

\begin{tabular}{llc}
\hline Person Obtaining Consent - Signature & Printed Name & Date
\end{tabular}




\section{Methodist \\ DALLAS MEDICAL CENTER}

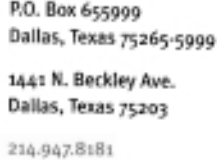

214.947 .8181

October 15,2007

Title: Anticipating Mothering for the Mexican Origin Women

Sponsor: Methodist Investigator Initiated Study

PI: Tony Patemiti, PhD, CCHt., RN

Sub-Investigator: Susan Baxley RN \& Mary Lou Bond, RN, PhD, FANN

IRB No. 2007.00.416.A

Re: Revised Informed Consent Version (10/10/07)

This is to inform you that I have reviewed and approved the above captioned revised consent form (version 10/10/07) changes inclusive of September 15, 2007 letter. This study was approved for a period of 12 months. The next continuing review is due on or before September 12, 2008. Please send your continuing review form to the IRB office at least 21 days before your study expires.

Expedited review approvals are submitted to the next IRB panel meeting to keep "all members advised of research proposals, which have been approved under the procedure." There is always the possibility the full board may make a change in the expedited review approvals that are submitted, and we will notify you if that occurs.

The next continuing review is due on or before September 12, 2008. Please send your continuing review form to the IRB office at least 21 days before your study expires.

The approval decision for the study is based on minimizing risk, acceptable risk-benefit ratio for subjects, equitable selection of subjects, confidentiality in the use of data ${ }^{1}$ and properly executed and documented informed consent.

You must fulfill all requirements of the IRB written procedures, including, but not limited to the following:

1. Conduct the research as required by the Protocol.

2. Use only the Consent Form approved by the Board.

Confidentiality in the use of data requires at a minimum: (a) not abstracting personal identifiers such as names, addresses, telephone numbers, e-mail addresses, of Social Security Numbers except when essential to an approved study protocol; (b) removing coded personal identifiers such as clinic or hospital numbers at the earliest stage of the research compatible with the study goals, such as after linking data from various sources together; (c) If coded personal identifiers much remain to combine with future data, these should be encrypted and not be the plain clinic or hospital numbers; and (d) the data files should be kept in a secure environment such as a locked cabinet or a properly secured computer file with password protection. 
3. If you provide Non-English speaking patients with a translation of the approved Consent Form in the patient's first language, it must be certified and approved by the IRB. The Board must approve the translated version.

4. Obtain pre-approval from the Board of any changes in the research activity (except when necessary to protect human subjects ;(HHS 45 CFR \& 46.103(b)(4); FDA 21 CFR \& 56.108(a)(3)); immediately report to the Board any such emergency changes for the protection of human subjects.

5. Within 5 days, report in writing to the Board the death, hospitalization, or serious Illness of any study subject enrolled in a Methodist IRB approved protocol that is or may be related to your study.

6. Within (5) days, promptly report to the Board any new information that might adversely affect the safety of the subjects or the conduct of the trial

7. Provide reports to the Board concerning the progress of the research, when requested.

8. Obtain pre-approval of study advertisements from the Board before use.

9. Conduct the informed consent process, without coercion or undue influence and provide the potential subject sufficient opportunity to consider whether or not to participate.

If you have any questions or need additional information, please contact the IRB office at (214) $947-2542$.

Sincerely,

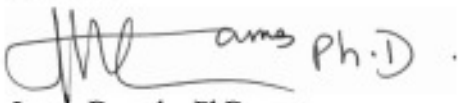

Jamds Dzandu, $\mathrm{PhD}$,

Methodist Institutional Review Board Interim Co-Chairman

1

Confidentiality in the use of data requires at a minimum: (a) not abstracting personal identifiers sach as names, addresses, telephone numbers, e-mail addresses, or Social Security Numbers except when essential to an approved stady protocol; (b) removing coded personal identifiers such as clinic or hospital numbers at the earliest stage of the research compatible with the study goals, such as after linking data from various sources together; (c) If coded personal identifiers much remain to combine with future data, these should be encrypted and not be the plain elinic or hospital numbers; and (d) the data files should be kept in a secure

environment such as a locked cabinet or a properly secured coniputer file with password protection. 
OEC 212007

APPROVED BY THE UTA - IRE The IRB approval for this consent

Document will expire on

\section{THE UNIVERSITY OF TEXAS}

DEC 202008

AT ARLINGTON

\section{INFORMED CONSENT}

PRINCIPAL INVESTIGATOR: Susan M. Baxley

TITLE OF PROJECT: Anticipating Mothering for the Mexican Origin Woman

This Informed Consent will explain about being a research subject in an experiment. It is important that you read this material carefully and then decide if you wish to be a volunteer.

\section{PURPOSE:}

The purpose(s) of this research study is/are as follows:

to explore the meaning of attachment, commitment, and preparation during pregnancy as an anticipatory phase of mothering for the Mexican onigin woman giving birth in the United States.

\section{DURATION}

The interview will take approximately 30-60 minutes. After the interview, we will meet again in person or by phane to review the interview summary (approximately 10-15 minutes), and once to review the final themes for the intent of what all the women meant to say (approximately 10-15 minutes and may be done by phone or in person.

There will be approximately 10-20 participants.

\section{PROCEDURES}

The procedures, which will involve you as a research subject, include:

1. Once you agree to be a part of the study, the researcher will interview you at that time or you may schedule a convenient time either in person or on the telephone. A Mexican origin nurse may be with the researcher at the time of the interview.

2. The interview will be audiotaped and last 30-60 minutes. You will be asked questions about your pregnancy and the baby. (A $\$ 25.00$ gift certificate to Target will be given at this time).

3. At another prearranged time, I will visit with you either in person or by telephone to review a summary of your interview for accuracy. A Mexican origin nurse may be available during the confirmation of the summary to allow you to add anything you might want. After the final review of all the women's interviews by the researcher, you will receive a copy of a summary of the study.

PRINCIPAL INVESTIGATOR: Susan M. Baxley

Page 1 of 4

Subject Initials 
TITLE OF PROJECT: Anticipating Mothering for the Mexican Origin Woman

\section{POSSIBLE RISKSIDISCOMFORTS}

The possible risks and/or discomforts of your involvement include:

During the interview process, you may experience possible psychological discomfort while talking about your foelings and the loss of confidentiality. After the study your consent and your interview will be kept separately and stored in a locked The only inconvenience is the time it will take for the interview and to meet to look at the summary of your interview.

\section{POSSIBLE BENEFITS}

The possible benefits of your participation are:

There are no direct benefits from participation. The Participant may gain satisfaction from the knowledge that they are making contributions which have the potential to assist health care workers provide culturally sensitive care.

\section{ALTERNATIVE PROCEDURES / TREATMENTS}

The alternative procedures / treatments available to you if you elect not to participate in this study are:

Your treatment in the clinic will not differ from the normal prenatal care that you have received or will continue to receive.

\section{CONFIDENTIALITY}

Every attempt will be made to see that your study results are kept confidential. A copy of the records from this study will be stored in School of Nursing at Center for Nursing Research at the University of Texas at Arlington for at least three (3) years after the end of this research. The results of this study may be published and/or presented at meetings without naming you as a subject. Although your rights and privacy will be maintained, the Secretary of the Department of Health and Human Services, the UTA IRB, and personnel particular to this research (individual or department) have access to the study records and will not be reviewed by the researcher or interviewer. Your medical records will be kept completely confidential according to current legal requirements. They will not be revealed unless required by law, or as noted above. The researcher and interviewer will not review your medical record.

DEC 212007

APPROVED BY THE UTA - IRB

The IRB approval for this consont

Document will expire on

DEC 202008

Last Revised mm/dd/yy

Subjoct lnitials

Page 2 of 4 
PRINCIPAL INVESTIGATOR: Susan M. Baxley

TITLE OF PROJECT: Anticipating Mothering for the Mexican Origin Woman

\section{COMPENSATION FOR MEDICAL TREATMENT:}

The University of Texas at Arlington (UTA) will pay the cost of emergency first aid for any injury that occurs as a result of your participation in this study. UTA will not pay for any other medical treatment. Claims against UTA or any of its agents or employees may be submitted according to the Texas Tort Claims Act (TTCA). These claims may be settled to the extent allowable by state law as provided under the TTCA., (Tex. Civ. Prac. \& Rem. Code, secs. 101.001, et seq.). For more information about claims, you may contact the Chairman of the Institutional Review Board of UTA at 817/272-1235.

You will receive a \$25.00 Target gift card and Certificate of Appreciation for your time to participate in this study. At the last visit to confirm the themes, you will receive the gift card and certificate as a thank you.

\section{FINANCIAL COSTS}

The possible financial costs to you as a participant in this research study are:

There are no financial costs to the participant except the time it takes to participate.

\section{CONTACT FOR QUESTIONS}

If you have any questions, problems or research-related medical problems at any time, you may call Susan Baxley at 214-505-9161, or Mary Lou Bond at 817-272-1271. You may call the Chairman of the Institutional Review Board at 817/272-1235 for any questions you may have about your rights as a research subject.

\section{VOLUNTARY PARTICIPATION}

Participation in this research experiment is voluntary. You may refuse to participate or quit at any time. If you quit or refuse to participate, the benefits to which you are otherwise entitled will not be affected. You may quit by calling Susan Baxley, whose phone number is 214-505-9161. You will be told immediately if any of the results of the study should reasonably be expected to make you change your mind about staying in the study.

\section{DEC 212000}

APPROVED BY THE UTA - IRB

The IRB approval for this consent

Last Revised mm/dd/yy

Document will expire on

Subject Initials

Page 3 of 4

DEC 202008 
PRINCIPAL INVESTIGATOR: Susan M. Baxley

TITLE OF PROJECT: Anticipating Mothering for the Mexican Origin Woman

By signing below, you confirm that you have read or had this document read to you. You will be given a signed copy of this informed consent document. You have been and will continue to be given the chance to ask questions and to discuss your participation with the investigator.

You freely and voluntarily choose to pe in this research project. PRINCIPAL INVESTIGATOR:

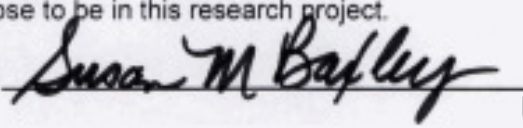

DATE

SIGNATURE OF VOLUNTEER

DATE

SIGNATURE OF PATIENT/LEGAL GUARDIAN (if applicable)

DATE

SIGNATURE OF WITNESS (if applicable)

DEC 212007

APPROVED BY THE UTA - IRB

The IRB approval for this consent

Document will expire on

DEC 202008

Last Revised mm/dd/yy

Subject Initials

Page 4 of 4 
Susan M. Baxley

February 22, 2008

Mary Lou Bond, Ph.D.

THE UNIVERSTY Nursing

Box 19407

CFTEXAS

AT ARLINGTON

Orice of Grant 8

Concract Services

Bor 19145

RE

Minor Modification Approval Letter

Title:

Anticipating mothering for the Mexican Origin Woman

IRB No.:

$2008.079 \mathrm{~s}$

200 E. Bonter Sc, Sules 216

Arlingten Teas

$76019-0145$

T817.272105

F $817.2 \mathrm{nsa09}$

Gos Home

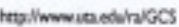

Epertacuturatirgen

hapilmeivadviopertie

The UT Arlington Institutional Review Board (UTA IRB) Chair (or designee) reviewed and approved the modification(s) to this protocol on February 22, 2008 in accordance with Title 45 CFR 46.110(b)(2). Therefore, you are authorized to conduct your research. The modification(s), indicated below, was deemed minor and appropriate for expedited review.

- Protocol modified for the interview to be done either during the initial visit with the subject or on the telephone and then verified with the individual by the Mexican origin nurse and the principal investigator.

Pursuant to Title 45 CFR 46.103(b)(4)(iii), investigators are required to, "promptly report to the IRB $a n y$ proposed changes in the research activity, and ensure that such changes in approved research, during the period for which IRB approval has already been given, are not initiated without IRB review and approval except when necessary to eliminate apparent immediate hazards to the subject."

The modification approval will additionally be presented to the convened board on March 11, 2008 for full IRB acknowledgment [45 CFR 46.110(c)]. All investigators and key personnel identified in the protocol must have documented Human Subjects Involved in Research (Tier II) Training or other UTA approved compliance education in the responsible conduct of human subject research on file with the UT Arlington Qffice of Research Administration Regulatory Services.

The UT Arlington Office of Research Administration appreciates your continuing commitment to the protection of human research subjects. Should you have questions or require further assistance, please contact Jan Parker by calling (817) 272-0867.

Sincerely,

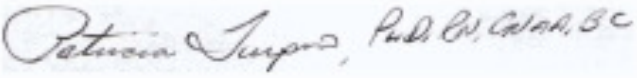

Patricia Turpin, PhD, RN, CNAA, BC

Associate Clinical Professor

UT Arlington IRB Chair 


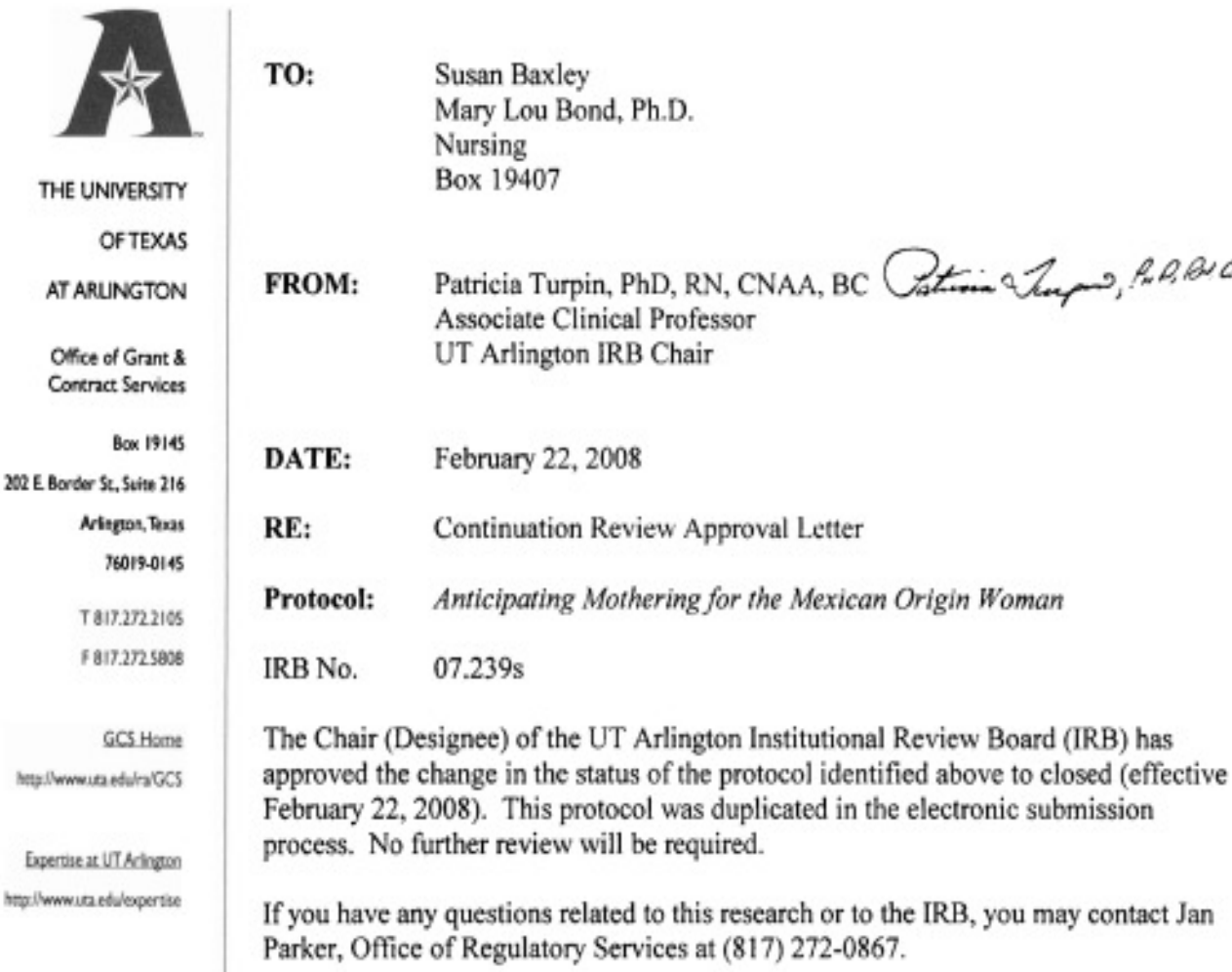




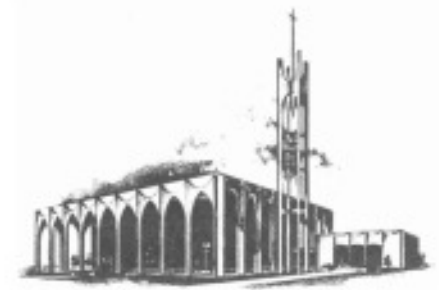

THE CHURCH OF SAINT MONICA

9933 MIDWAY ROAD DALLAS, TEXAS 75220 PHONE 214-358-1453

FAX 214-351-1887

January 12,2008

The University of Texas at Arlington

University Institutional Review Board

Arlington, Texas

To Whom It May Concern:

Ms. Susan M. Baxley, RNC, MS, Ph.D Candidate. is undertaking a scientific study,

"Anticipating Mothering for the Mexican Woman."

As the pastor of St. Monica Parish and its Mission of San Juan Diego, we are pleased to cooperate with her in this study, specifically by soliciting participation of qualified women in the project.

We wish her success in her study and look forward to the results.

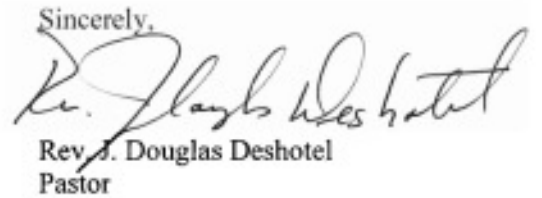




\section{Los Barrios Unidos Community Clinic, Inc.}

February 4, 2008

Susan M. Baxley RNC, MS, PhD candidate

$1114 \mathrm{~N}$. Windomere Avenue

Dallas, Texas 75208

Re: Research at LBUCC

Dear Ms. Baxley:

Please be advised that you hereby have permission to approach patients at Los Barrios Unidos Community Clinic to recruit women that are pregnant to participate in a study. conducted by you, entitled "Anticipating Mothering for the Mexican Origin Woman." understand that you are conducting this research as a $\mathrm{Ph} . \mathrm{D}$. candidate at The University of Texas at Arlington. Please guarantee that the patients understand fully the purpose of the study and that they give full consent. I understand the patients' participation will be interview-based.

Please to consult Sylvia Urueta, LBUCC OB/Gyn Nurse Practitioner, on all issues related to these patients. I wish you well on a successful project.

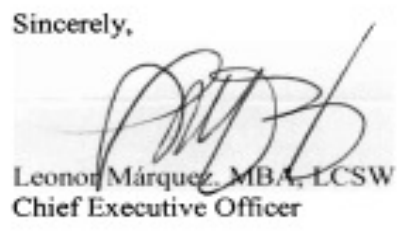

CC: Sylvia Ureta, RNC, WHNP

809 Singleton Blvd. • Dallas, Texas 75212 - (214) $651-8739$

Administration (214) 571-6132 - Fax (214) 651-9514

www.losbarriosunidos.org 
APPENDIX F

AUDIT TRAIL FOR ANALYSIS 


\begin{tabular}{|c|c|c|c|c|c|c|c|c|}
\hline code \# & $\begin{array}{l}\text { interview } \\
\text { date }\end{array}$ & site & $\begin{array}{c}\text { phone/ in- } \\
\text { person }\end{array}$ & interviewer & transcription & $\begin{array}{c}\text { independent } \\
\text { verifier }\end{array}$ & analysis & $\begin{array}{c}\text { Independent } \\
\text { coder }\end{array}$ \\
\hline 1 & none & site \#1 & & & & & & \\
\hline 2 & none & site \#1 & & & & & & \\
\hline 3 & $2 / 26 / 2008$ & site \#2 & in person & researcher & researcher & E. P. & $x$ & \\
\hline 4 & 2/19/2008 & site \#2 & phone & $\begin{array}{c}\text { M.O. } \\
\text { interviewer/res } \\
\text { earcher }\end{array}$ & researcher & E. P. & $\mathrm{x}$ & \\
\hline 5 & & site \#2 & phone & & & & & \\
\hline 6 & $3 / 5 / 2008$ & site \#2 & in person & researcher & researcher & E. P. & $x$ & \\
\hline 8 & & site \#2 & & researcher & researcher & & & \\
\hline 9 & $4 / 11 / 2008$ & site \#2 & in person & researcher & researcher & E. P. & $x$ & D. B. \\
\hline 10 & $4 / 11 / 2008$ & site \#2 & in person & researcher & researcher & E. P. & $x$ & D. B. \\
\hline 11 & $4 / 1 / 2008$ & site \#2 & in person & researcher & researcher & E. P. & $x$ & \\
\hline 12 & & site \#2 & in person & researcher & & & & \\
\hline 13 & & site \#2 & in person & researcher & & & & \\
\hline 14 & $4 / 11 / 2008$ & site \#2 & in person & researcher & researcher & E. P. & $x$ & \\
\hline 15 & $4 / 11 / 2008$ & site \#2 & in person & researcher & researcher & E. P. & $x$ & \\
\hline 16 & & site \#2 & in person & researcher & & & & \\
\hline $7 \& 17$ & $5 / 16 / 2008$ & site \#2 & in person & researcher & researcher & E. P. & $x$ & \\
\hline 18 & & site \#2 & in person & researcher & & & & \\
\hline 19 & & site \#2 & in person & researcher & & & & \\
\hline 20 & $6 / 11 / 2008$ & site \#2 & in person & researcher & researcher & E.P & $x$ & \\
\hline
\end{tabular}


APPENDIX G

CHARACTERISTICS OF SUBJECTS INTERVIEWED 


\begin{tabular}{|c|c|c|c|c|c|c|c|c|c|c|c|}
\hline code \# & initials & age & gest.age & $\begin{array}{c}\text { country } \\
\text { origin }\end{array}$ & lived & $\begin{array}{l}\text { lang. } \\
\text { spok }\end{array}$ & work & school & married & live with & $\begin{array}{c}\text { partner } \\
\text { involved }\end{array}$ \\
\hline 3 & S.G & 20 & 30 & Mex- & born here & Eng. & yes & yes & no & aunt & \begin{tabular}{|l} 
yes at time \\
interview
\end{tabular} \\
\hline 4 & F.M & 18 & 26 & Mex- & born here & both & no & no & no & parents & yes \\
\hline & & & & & & & & & & & \\
\hline 6 & A. M. & 18 & 30 & Mex- & $12 \mathrm{yrs}$ & Span & no & graduated & no & parents & distant \\
\hline 9 & C.G. & 18 & 25 & Mex- & 6 vrs & Snan & ves & no & no & mom & $\ln$ \\
\hline 10 & M.G. & 19 & 23 & Mex- & $181 / 2$ & Span & no & $\begin{array}{l}\text { to start } \\
\text { college }\end{array}$ & yes & $\begin{array}{l}\text { mom\& } \\
\text { husband }\end{array}$ & yes \\
\hline 11 & D. G. & 18 & 23 & Mex- & 9 & Span & yes & yes & no & mom & yes \\
\hline 14 & M. G. & 23 & 29 & Mex- & 6 & both & yes & no & no & aunt & no \\
\hline 15 & E. M. & 18 & 28 & Mex- & 18 & both & no & yes-12th & yes & $\begin{array}{l}\text { parents } \\
\text { \&husband }\end{array}$ & yes \\
\hline $7 \& 17$ & & 18 & 29 & Mex- & 11 & Span & no & no & yes & husband & yes \\
\hline 20 & D. H. & 18 & 25 & Mex- & 13 & both & no & no & common & husband & \\
\hline
\end{tabular}




\section{REFERENCES}

Alhusen, J. L. (2008). A literature update on maternal-fetal attachment. JOGNN, 37(3), 315-328.

Altarriba, J. \& Santiago-Rivera, Azara L. (1994). Current perpectives on using linguistic and cultural factors in counseling the Hispanic client. Professional Psychology: Research and Practice, 25 (388-397).

Amaro, H. (1988). Women in the Mexican-American community: Religion, culture, and reproductive attitudes and experiences. Journal of Community Psychology, 16, 6-20.

Andrews, M. (2003). Theoretical foundations of transcultural nursing. In M. Andrews, \& J. Boyle (Ed.), Transcultural concepts in nursing care (pp. 3-14). Philadelphia: Lippincott Williams and Wilkins.

Auerswald, C., Greene, K., Minnis, A. D., I., Ellen, J. Podian, N. (2004). Qualitative assessment of venues for purposive sampling of hard-to-reach youth an illustration in a Latino community. Sexually Transmitted Diseases, 31, 133-138.

Balcazar, H., Krull, J. L., \& Peterson, G. (2001). Acculturation and family functioning are related to health risks among pregnant Mexican American women. Behavioral Medicine, 27(62-70).

Becerra, J. E., Hogue, C. J. R., Atrash, H. K., \& Perez, N. (1991). Infant mortality among Hispanics: A portrait of heterogeneity. Journal of the American Medical Association, 265, 217-221. 
Becerra, R. M., \& de Anda, D. (1984). Pregnancy and motherhood among Mexican American adolescents. Health and Social Work, 9(2), 106-123.

Benedek, T. (1956). Psychobiological aspects of mothering. The American Journal of Orthopsychiatry, 26(2), 272-278.

Bernstein, R. (2006). Nation's population one-third minority. Retrieved March 12, 2007 from http://www.census.gov.

Bernstein, R. (2008). An older and more diverse nation by midcentury. Retrieved September 1, 2008 from http://www.census.gov.

Berry, A. B. (1999). Mexican American women's expressions of the meaning of culturally congruent prenatal care. Journal of Transcultural Nursing, 10(3), 203-212.

Berry, J. W. (2002). Conceptural approaches to acculturation . In K. M. Chun, P. B. Organista, \& G. Marin (Eds.), Acculturation advances in theory, measurement, and applied research (pp. 17-37). Washington, DC: American Psychological Association.

Bohannan, P. (1992). We, the alien An introduction to cultural anthropology. Prospect Heights, Illinois: Waveland Press, Inc.

Bronfenbrenner, U. (1979). The ecology of human development: Experiment by nature and design. Cambridge, MA: Harvard University Press.

Callister, L. C. (1995). Cultural meaning of childbirth. Journal Obstetrical Gynecological and Neonatal Nursing, 24(4), 327-331.

Callister, L. C. (2001). Culturally competent care of women and newborns: Knowledge, attitude, and skills. JOGNN, 30(2), 209-215. 
Callister, L. C., \& Vega, R. (1998). Giving birth: Guatemalan women's voices. Journal of Obstetric, Gynecologic, and Neonatal Nursing, 27(3), 289-295.

Cannella, B. L. (2005). Maternal-fetal attachment: An integrative review. Journal of Advanced Nursing, 50(1), 60-68.

Chun, K. M., \& Akutsu, P. D. (2002). Acculturation among ethnic minority families. In K. M. Chun, P. B. Organista, \& G. Marin (Eds.), Acculturation: Advances in theory, measurement, and applied research (pp. 95-119). Washington DC: American Psychological Association.

Cohen, J. (1992). Janforum: Leininger's culture care theory of nursing. Journal of Advanced Nursing, 17, 1149.

Colman, A. D., \& Colman, L. L. (1973). Pregnancy: The psychological experience. New York: The Seabury Press.

Cranley, M. S. (1981). Development of a tool for the measurement of maternal attachment during pregnancy. Nursing Research, 30(5), 281-284.

Cummins, L., \& Scrimshaw, S. \&. E. P. (1988). Views of cesarean birth among primiparous women of Mexican origin in Los Angeles . Birth, 15, 164-170.

Cuéllar, I., Arnold, B., \& Maldonado, R. (1995). Acculturation rating scale for Mexican AmericansII: A revision of the original ARSMA scale. Hispanic Journal of Behavioral Sciences, 17(3), 275-304.

Cutcliffe, J. R. \& McKenna, H. P. (1999). Establishing the credibility of qualitative research findings: The plot thickens. Journal of Advanced Nursing, 30, 374-380. 
Deci, E. L., Vallerand, R. J., Pelletier, Luc G., Ryan, R. M. (1991). Motivation and education: The self-determination perspective. Educational Psychologist, 26 (3 \& 4), 325-346.

Dingley, C. E., Roux, G., Bush, H.A. (2000). Inner strength: A concept analysis. The Journal of Theory Construction \& Testing, 4 (2), 30-35.

Deutsch, H. (1945). Motherhood, motherliness, and sexulaity. Psychology of Women New York: Grune \& Stratton.

Domian, E. W. (2001). Cultural practices and social support of pregnant women in a northern New Mexico community. Journal of Nursing Scholarship, 33(4), 331-336.

Engle, P. L., Scrimshaw, S. M., Zambrana, R. E., \& Dunkel-Schetter, C. (1990). Prenatal and postnatal anxiety in Mexican women giving birth in Los Angeles. Health Psychology, 9(3), 285-299.

Florsheim, P., Sumida, E., McCann, C., Winstanley, M., Fukui, R., Seefeldt, T. et al. (2003). The transition to parenthood among young African American and Latino couples: Relational predictors of risk for parental dysfunction. Journal of Family Psychology, 17(1), 65-79.

Giger, J., \& Davidhizar, R. (1999). Transcultural nursing assessment \& intervention. St. Louis: Mosby, Inc.

Gilliss, C. L., Lee, K. A., Gutierrez, Y., Taylor, D., Beyene, Y., Neuhaus, J. et al. (2001). Recruitment and retention of healthy minority women into community-based longitudinal research. Journal of Women's Health, 10(1), 77-85.

Gonzales, F. (2008). Hispanic women in the United States, 2007. Pew Hispanic Center. Retrieved on May 14, 2008, from www.pewhispanic.org. 
Guendelman, S., \& Abrams, B. (1995). Dietary intake among Mexican American women:

Generational differences and a comparison with White non-Hispanic women. American Journal of Public Health , 85, 20-25.

Guendelman, S., Malin, C., Herr-Harthorn, B., \& Vargas, P. N. (2001). Orientation to motherhood and male partner support among women in Mexico and Mexican-origin women in the United States. Social Science \& Medicine, 52, 1805-1813.

Harris, K. J., Ahluwalia, J. S., Catley, D., Okuyemi, K. S., Mayo, M. S., \& Resnicow, K. (2003). Successful recruitment of minorities into clinical trials: The Kick It at Swope project. Nicotine \& Tobacco Research, 5(4), 575-584.

Jones, M. E., \& Bond, M. L. (1999).Predictors of birth outcome among hispanic immigrant women. Journal Nursing Care Quality, 14(1), 56-62.

Keefe, S. M. (1980). Acculturation and the extended family among urban Mexican Americans. In A. M. Padilla (Ed.), Acculturation: Theory, models, and some new findings (pp. 85-110). Boulder, CO: Westview.

Koniak-Griffin, D. (1993 ). Maternal role attainment. Image: The Journal of Nursing Scholarship, $25,257-262$.

Koniak-Griffin, D., Logsdon, M. C., Hines-Martin, V., \& Turner, C. C. (2006). Contemporary mothering in a diverse society. JOGNN, 35(5), 671-677.

Kreps, G., \& Kunimoto, E. (1994). Effective communication in multicultural health care settings. Thousand Oaks: Sage Publications. 
Laganó, K. (2003). Come bien, camina y no se preoccupe-Eat right, walk, and do not worry: Selective biculturalism during pregnancy in a Mexican American community. Journal of Transcultural Nursing, 14(2), 11-.

Langaná, K., \& Gonzalez-Rameriz, L. (2003). Mexican Americans. In P. Hill, J. Lipson, \& A. Meleis (Eds.), Caring for women cross-culturally (pp. 218-235). Philadelphia: F. A. Davis Co.

Lederman, R. P. (1984). Pschosocial adaptation in pregnancy: Assessment of seven dimensions of maternal development. Englewood Cliffs, NJ: Prentice-Hall, Inc.

Lederman, R. P. (1996). Psychosocial adaptation in pregnancy. New York: Springer Publishing Company.

Leifer, M. (1980). Psycholgical effect of motherhood: A study of first pregnancy. New York: Prager.

Leininger, M. (1995). Transcultural nursing: Concepts, theories, research \& practice. New York: McGraw-Hill.

Leininger, M. Ed. (1976). Transcultural health care issues and conditions. Philadelphia: F. A. Davis.

Liamputtong, P., \& Ezzy, D. (2005). Qualitative research methods. Victoria, Australia: Oxford University Press.

Lincoln, Y. S., \& Guba, E. G. (1985). Naturalistic inquiry. Beverly Hills, CA: Sage.

Lindgren, K. (2001). Relationships among maternal-fetal attachment, prenatal depression and health practices in pregnancy. Research in Nursing and Health, 24, 203-217. 
Lipson, J. G., \& Meleis, A. I. (1999). Research with immigrants and refugees. In S. L. F. J. L. F. S. A. S. Hinshaw (Eds.), Handbook of clinical nursing research (pp. 87-106). Thousand Oaks, CA: Sage Publications.

Lydon, J.E. \& Zanna, M. P. (1990). Commitment in the face of adversity: A value-affirmation approach. Journal of Personality and Social Psychology, 58 (6), 1040-1047.

Mahon, J., McFarlane, J., \& Golden, K. (1991). De madres a madres: A community partnership for health. Public Health Nursing, 8(1), 15-19.

Marin, G., \& Gamba, R. J. (2002). Acculturation and changes in cultural values. In K. M. Chun, P. B. Organista, \& G. Marin (Eds.), Acculturation: Advances in theory, measurement, and applied research (pp. 83-93). Washington, D C: American Psychological Association.

Marin, G., \& Marin, B. V. (1991). Research with Hispanic populations. Newbury Park: Sage Publications.

Mattson, S. (2003). Caring for Latino women. AWHONN Lifelines, 7(3), 258-260.

Mayan, M. J. (2001). An introduction to qualitative methods: $A$ training module for students and professionals. Alberta, Canada: Qual Institute Press.

McBride, A. B., \& Shore, C. P. (2001). Women as mothers and grandmothers. Annual Review of Nursing Research, 19, 63-85.

Mead, G. H. (1934). Mind, self, and society. Chicago: The University of Chicago Press.

Mead, M., \& Newton, N. (1967). Cultural patterning of perinatal behavior. In S. A. Richardson, \& A. F. Guttmacher (Ed.), Childbearing=Its Social and Psychological Aspects . Baltimore: Williams and Wilkins Co. 
Meleis, A. (2003). Theoretical considerations of health care for immigrant and minority women. In P. Hill, J. Lison, \& A. Meleis (Eds.), Caring for women cross-culturally (pp. 1-10). Philadelkphia: F. A. Davis Company.

Meleis, A. I., Douglas, M. K., Eribes, C., Shih, F., \& Messias, D. K. (1996). Employed Mexican women as mothers and partners: valued, empowered and overloaded. Journal of Advanced Nursing, 23, 82-90.

Mercer, R. T. (1980). Teenage motherhood: The first year. Journal of Obstetric, Gynecologic, and Nenonatal Nursing, 9, 16-27.

Mercer, R. T. (1981). A theoretical framework for studying factors that impact on the maternal role. Nursing Research, 30(2), 73-77.

Mercer, R. T. (1985). The process of maternal role attainment over the first year. Nursing Research, 34, 198-204.

Mercer, R. T. (1986). Predictors of maternal role attainment at one year postbirth. Western Journal of Nursing Research, 8(1), 9-32.

Mercer, R. T. (1995). Becoming a mother: Research on maternal identity from Rubin to the present. New York: Springer.

Mercer, R. T. (2004). Becoming a mother versus maternal role attainment. Journal of Nursing Scholarship, 36, 226-232.

Mercer, R. T. (2006). Nursing support of the process of becoming a mother. JOGNN, 35(5), 649651. 
Mercer, R. T., \& Ferketich, S. L. (1990). Predictors of parental attachment during early parenthood. Journal of Advanced Nursing, 15, 268-280.

Mercer, R. T., \& Ferketich, S. L. (1995). Experienced and inexperienced mothers' maternal competence during infancy. Research in Nursing and Health, 18, 333-343.

Mercer, R. T. \& Walker, L. O. (2006). A review of nursing interventions to foster becoming a mother. JOGNN, 35(5), 568-582.

Miller, T. (2005). Making sense of motherhood: A narrative approach. Cambridge, UK: Cambridge University Press.

Morse, J. M. \& Richards, L. (2002). Readme First for a User's Guide to Qualitative Methods. Thousand Oaks: Sage Publications.

Mosenthal, P. (2001). Language and thought. Theory into Practice, 14, 306-311.

Muller, M. E. (1990). The development and testing of the Muller prenatal attachment inventory. Unpublished doctoral dissertation, University of California, San Francisco.

Muller, M. E. (1992). A critical review of prenatal attachment research. Scholarly Inquiry for Nursing Practice: An International Journal, 6(1), 5-26.

Muller, M. E. (1993). Development of the prenatal attachment inventory. Western Journal of Nursing Research, 15(2), 199-215.

Müller, M. E. (1996). Prenatal and postnatal attachment: A modest correlation. JOGNN, 25, 161166.

Murphy, S. T. (2008). Preparation is everything. World of Irish Nursing \& Midwifery, 16(5), 30-31. 
Negy, C. (1993). Anglo- and Hispanic-Americans' performance on the family attitude scale and its implications for improving measurements of acculturation. Psychological Reports, 73, $1211-1217$.

Nelson, A. M. (2003). Transition to motherhood. JOGNN, 32(4), 465-477.

Niska, K. J. (1999). Family nursing interventions: Mexican American early family formation. Nursing Science Quarterly, 12(4), 335-340.

Oakley, A. (1979). Becoming a mother. New York: Schocken Books.

Office of Minority Health. (2001). National standards for culturally and linguistically appropriate services in health care. Washington, DC: U.S. Department of Health and Human Services.

Pascoe, J. M., Kototailo, P. K., \& Broekhuizen, F. F. (1995). Correlates of multigravida women's binge drinking during pregnancy. Archives of Pediatric and Adolescent Medicine, 149, 1325-1329.

Patton, M. Q. (2002). Qualitative research \& evaluation methods. Thousand Oaks, CA: Sage Publications.

Pew Hispanic Center. (2006). Hispanics at mid-decade. (Report No. Table 9 Retrieved March 4, 2007 from www.pewhispanic.org).

Pew Hispanic Center. (2002). U.S.-born Hispanics increasingly drive population developments. Pew Hispanic Fact Sheet, Retrieved March 4, 2007 from www.pewhispanic.org.

Pew Research Center. (2006).U. S. Population Projections: 2005-2050, Pew Hispanic Center Report. Retrieved September 1, 2008 from http:Ilwww.pewhispanic.org/reports/. 
Pitney, W. A. (2004). Strategies for establishing trustworthiness in qualitative research. Athletic Therapy Today, 9(1), 26-28.

Porter, C. L., \& Hsu, H. C. (2003). First-time mothers' perceptions of efficacy during the transition to motherhood: Links to infant temperament. Journal of Family Psychology, 17(1), 54-64.

Priel, B., \& Besser, A. (2001). Bridging the gap between attachment and object relations theories: A study of the transition to motherhood. British Journal of Medical Psychology, 74, 85100.

Purnell, L. D. (2008). Transcultural diversity and health care. In L. D. Purnell \& B. J. Paulanka (Eds.), Transcultural health care: A culturally competent approach ( $3^{\text {rd }}$ ed., pp. 1-18). Philadelphia: F. A. Davis Company.

Purnell, L. D. (2008).The Purnell model for cultural competence. In L. D. Purnell \& B. J. Paulanka (Eds.), Transcultural health care: A culturally competent approach ( $3^{\text {rd }}$ ed., pp.19-55). Philadelphia: F. A. Davis Company.

Redfield, R., Linton, R., \& Herskovits, M. J. (1936). Memorandum on the study of acculturation. American Anthropologist, 38(1), 149-152.

Reid, J. (2007). Centering Pregnancy®: A model for group prenatal care. Nursing for Women's Health, 11(4), 382-388.

Rubin, R. (1967). Attainment of the maternal role Part 11 models and referrants. Nursing Research, 16(4), 342-346.

Rubin, R. (1967). Attainment of the maternal role Part I. processes. Nursing Research, 16(3), 237-245. 
Rubin, R. (1984). Maternal identity and the maternal experience. New York: Springer Publishing Company.

Rubin, R. (1975). Maternal tasks in pregnancy. Maternal-Child Nursing Journal, 4, 143-153.

Rueschenberg, E., \& Buriel, R. (1989). Mexican American family functioning and acculturation: A family systems approach. Hispanic Journal of Behavioral Sciences, 11, 232-244.

Ryder, A. G., Alden, L. E., \& Paulhus, D. L. (2000). Is acculturation unidimensional or bidemensional? Journal of Personality and Social Psychology, 79, 49-65.

Sagrestano, L. M., Feldman, P., Rini, C. K., Woo, G., \& Dunkel-Schetter, C. (1999). Ethnicity and social support during pregnancy. American Journal of Community Psychology, 27(6), 869-898.

Sandelowski, M., Davis, D. H., \& Harris, B. G. ( 1989). Artful design: Writing the proposal for research in the naturalist paradigm. Research in Nursing \& Health, 12(2), 77-84.

Sandelowski, M. (2000). Focus on research methods Whatever Happened to Qualitative Description? Research in Nursing \& Health, 23, 334-340.

Sank, J. C. (1991). Factors in the prenatal period that affect parental role attainment during the postpartum period in black American mothers and fathers. Unpublished doctoral dissertation, University of Texas at Austin, Austin, Texas.

Sarbin, T. R. (1954). Role theory. In G. Lindzey (Ed.), Handbook of social psychology (pp. 223258). Reading, Mass.: Addison-Wesley Publishing Co.

Sawyer, L. M. (1999). Engaged mothering: The transition to motherhood for a group of African American women. Journal of Transcultural Nursing, 10(1), 14-21. 
Shereshesky, P. M., \& Yarrow, L. J. (1973). Psychological aspects of a first pregnancy and early postnatal adaptation. New York: Raven Press.

Sherwen, L. N. (1987). Psychosocial dimensions of the pregnant family. New York: Springer Publishing Company.

Siatkowski, A. A. (2007). Hispanic acculturation: A concept analysis. Journal of Transcultural Nursing, 18(4), 316-323.

Siddiqui, A., Hagglof, B., \& Eisemann, M. (1999). An exploration of prenatal attachment in Swedish expectant women. Journal of Reproductive and Infant Psychology, 17(4), 369380.

Siddiqui, A., \& Hagglof, B. (2000). Does maternal prenatal attachment predict postnatal motherinfant interaction. Early Human Development, 59, 13-25.

Smith, J. A. (1999). Towards a relational self: Social engagement during pregnancy and psychological preparation for motherhood. British Journal of Social Psychology, 38, 409426.

Social Science Research Council. (1954). Acculturation: An exploratory formulation. American Anthropologist, 56(6), 973-1000.

Spector, R. (2004). Cultural diversity in health and illness. Upper Saddle River, N. J.: Pearson Hall.

Spradley, J. P. (1979). The ethnographic interview. New York: Holt, Pinehart and Winston.

Svensson, J., Barclay, L., \& Cooke, M. (2006), The concerns and interests of expectant and new parents: Assessing learning needs. The Journal of Perinatal Education, 15(4), 18-27. 
Svensson, J., Barclay, L., \& Cooke, M. (2007), Antenatal education as perceived by health professionals, The Journal of Perinatal Education, 16(1), 9-15.

Thornton, R., \& Nardi, P. M. (1975). The dynamics of role acquisition . American Journal of Sociology, $80,870-885$.

Tobin, G. A. \& Begley, C. M. (2004). Methodological rigour within a qualitative framework, Journal of Advanced Nursing, 48, 388-396.

U. S. Census Bureau. (2007). Population profile of the United States: Dynamic version: Race and Hispanic origin in 2005.) Retrieved September 13, 2008 from http://www.census.gov/population/www/pop-profile/profiledynamic.html.

Walker, L. O., Crain, H., \& Thompson, E. (1986). Maternal role attainment and identity in the postpartum period: Stability and change. Nursing Research, 35(2), 68-71.

Walker, L. O., Crain, H., \& Thompson, E. (1986). Mothering behavior and maternal role attainment during the postpartum period. Nursing Research, 35(6), 322-325.

Zoucha, R., \& Zamarripa, C. A. (2008). People of Mexican heritage. In L. D. Purnell, \& B. J. Paulanka (Eds.), Transcultural Health Care: A Culturally Competent Approach (3rd ed., pp. 309-324). Philadelphia: F. A. Davis Company. 


\section{BIOGRAPHICAL INFORMATION}

As a nurse, the author has practiced nursing for 40 years specializing in maternal-infant health. Her expertise in education and mentoring of patients, staff, and students led her to pursue a PhD in nursing with a research focus on Mexican origin women becoming mothers. She is interested in working with women of diverse cultures, understanding how we can best work together to improve the health of the next generation of children.

As a maternal/infant nurse with experience as an educator and program director, she has the expertise to teach and mentor others using special projects and creative program designs to achieve desired results. While working in the Center for Nursing Research, at the University of Texas at Arlington as a graduate research assistant, she served as project manager for several studies conducted in the School of Nursing. She also contributed to the study, Missing in Texas by transcribing data and coding focus groups and a contributing author to several publications derived from the study. The Ferne C. Newman Kyba Fellowship assisted in the completion of this dissertation. She is a co-author and Southern Nursing Research Society winner of the podcast "What is a Nurse Scientist", for the Council for the Advancement of Nursing Science podcast competition.

Future goals consist of becoming a proficient faculty member, teaching in the graduate program, and developing as a nurse scientist focusing on research with Mexican origin women to understand their needs and goals for their children. Developing community partnerships with clinics, churches, and families to promote culturally appropriate interventions is a priority for research and decreasing health disparities. 